\title{
Topological full groups of one-sided shifts of finite type
}

\author{
Hiroki Matui \\ Graduate School of Science \\ Chiba University \\ Inage-ku, Chiba 263-8522, Japan
}

\begin{abstract}
We explore the topological full group $[[G]]$ of an essentially principal étale groupoid $G$ on a Cantor set. When $G$ is minimal, we show that $[[G]]$ (and its certain normal subgroup) is a complete invariant for the isomorphism class of the étale groupoid $G$. Furthermore, when $G$ is either almost finite or purely infinite, the commutator subgroup $D([[G]])$ is shown to be simple. The étale groupoid $G$ arising from a onesided irreducible shift of finite type is a typical example of a purely infinite minimal groupoid. For such $G,[[G]]$ is thought of as a generalization of the Higman-Thompson group. We prove that $[[G]]$ is of type $\mathrm{F}_{\infty}$, and so in particular it is finitely presented. This gives us a new infinite family of finitely presented infinite simple groups. Also, the abelianization of $[[G]]$ is calculated and described in terms of the homology groups of $G$.
\end{abstract}

\section{Contents}

1 Introduction 2

2 Preliminaries 5

3 A spatial realization theorem 6

4 Simplicity of commutator subgroups 12

4.1 Almost finite groupoids . . . . . . . . . . . . . . . . 12

4.2 Purely infinite groupoids . . . . . . . . . . . . . . . . 15

5 Surjectivity of the index map

6 Groupoids from shifts of finite type 21

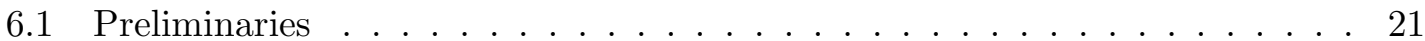

6.2 Equivalence between clopen subsets . . . . . . . . . . . . . . . . 24

6.3 The Haagerup property . . . . . . . . . . . . . . . . . 25

6.4 Kernel of the index map . . . . . . . . . . . . . . . . . 27

6.5 Finiteness properties . . . . . . . . . . . . . . . . . . . . . . . . . . . . . .

6.6 A presentation for $[[G \mid Y]] \ldots \ldots \ldots \ldots \ldots \ldots \ldots$

6.7 Examples . . . . . . . . . . . . . . . . . . . 45 


\section{Introduction}

We study various properties of topological full groups of topological dynamical systems on Cantor sets. H. Dye [11, 12] introduced the notion of full groups for ergodic measure preserving actions of countable groups and proved that the full group is a complete invariant of orbit equivalence. The study of full groups in the setting of topological dynamics was initiated by T. Giordano, I. F. Putnam and C. F. Skau [17]. For a minimal action $\varphi: \mathbb{Z} \curvearrowright X$ on a Cantor set $X$, they defined several types of full groups and showed that these groups completely determine the orbit equivalence class, the strong orbit equivalence class and the flip conjugacy class of $\varphi$, respectively.

The notion of topological full groups was later generalized to the setting of essentially principal étale groupoids $G$ on Cantor sets in [31]. Étale groupoids (called $r$-discrete groupoids in [37]) provide us a natural framework for unified treatment of various topological dynamical systems. The topological full group $[[G]]$ of $G$ is a subgroup of $\operatorname{Homeo}\left(G^{(0)}\right)$ consisting of all homeomorphisms of $G^{(0)}$ whose graph is 'contained' in the groupoid $G$ as a compact open subset (see Definition 2.1). From an action $\varphi$ of a discrete group $\Gamma$ on a Cantor set $X$, we can construct the étale groupoid $G_{\varphi}$, which is called the transformation groupoid. The topological full group $\left[\left[G_{\varphi}\right]\right]$ of $G_{\varphi}$ is the group of $\alpha \in \operatorname{Homeo}(X)$ for which there exists a continuous map $c: X \rightarrow \Gamma$ such that $\alpha(x)=\varphi_{c(x)}(x)$ for all $x \in X$. Another important class of étale groupoids is the AF groupoids. The terminology AF comes from $C^{*}$-algebra theory and means approximately finite. AF groupoids have played a crucial role in the study of orbit equivalence for minimal $\mathbb{Z}^{N}$-actions ([16]). The étale groupoids $G$ arising from one-sided irreducible shifts of finite type $(X, \sigma)$ have been also studied by many people. The groupoid $C^{*}$-algebras $C_{r}^{*}(G)$ are called Cuntz-Krieger algebras $([10])$. V. V. Nekrashevych [34] observed that $[[G]]$ is naturally isomorphic to the Higman-Thompson group $V_{n, 1}$ when $(X, \sigma)$ is the full shift over $n$ symbols (see Remark 6.3). Thus, the groups $[[G]]$ for shifts of finite type are regarded as a generalization of $V_{n, 1}$. For two étale groupoids $G_{1}$ and $G_{2}$ arising from one-sided irreducible shifts of finite type, K. Matsumoto [29] has recently proved that the topological full groups $\left[\left[G_{1}\right]\right]$ and $\left[\left[G_{2}\right]\right]$ are isomorphic as groups if and only if $G_{1}$ and $G_{2}$ are isomorphic as étale groupoids. This is analogous to the above mentioned result for minimal $\mathbb{Z}$-actions. Furthermore, Matsumoto 27] gave a sufficient condition on the subshifts under which the corresponding groupoids are isomorphic (see Theorem [6.2). Clearly the group $[[G]]$ embeds in the Leavitt path algebra associated with $(X, \sigma)$. This may indicate possible connections to the study of Leavitt path algebras.

In the present paper, we first extend the results of [17, 29]. More precisely, for given essentially principal minimal étale groupoids $G_{1}$ and $G_{2}$ on Cantor sets and normal subgroups $\Gamma_{i} \subset\left[\left[G_{i}\right]\right]$ containing the commutator subgroups $D\left(\left[\left[G_{i}\right]\right]\right)$, we will show that any isomorphism between $\Gamma_{1}$ and $\Gamma_{2}$ is realized by a homeomorphism between the unit spaces

$G_{i}^{(0)}$ (Theorem 3.5 and Proposition 3.6). This means that if $\Gamma_{1}$ and $\Gamma_{2}$ are isomorphic as groups, then $G_{1}$ and $G_{2}$ are isomorphic as étale groupoids (Theorem 3.9). In particular, $\left[\left[G_{i}\right]\right]$ (or $D\left(\left[\left[G_{i}\right]\right]\right)$ ) are isomorphic to each other if and only if $G_{i}$ are isomorphic to each other (Theorem [3.10). In other words, we can say that the topological full group $[[G]]$ (and its commutator subgroup $D([[G]])$ ) 'remembers' the étale groupoid $G$. We remark that similar results are also obtained by S. Bezuglyi and K. Medynets [1, 33].

In Section 4 we study simplicity properties of $[[G]]$. The notion of almost finite 
groupoids was introduced in [31. Almost finiteness is a weak version of approximate finiteness. The transformation groupoids of free actions of $\mathbb{Z}^{N}$ are known to be almost finite ([31, Lemma 6.3]). We show that if $G$ is almost finite and minimal, then the commutator subgroup $D([[G]])$ is simple (Theorem 4.7). This is a generalization of [30, Theorem 4.9] and [1, Theorem 3.4 (2)] for minimal $\mathbb{Z}$-actions. We also present a new class of étale groupoids, namely purely infinite groupoids. The definition of pure infiniteness is inspired by the work of M. Rørdam and A. Sierakowski [39] (see Definition 4.9). Transformation groupoids arising from $n$-filling actions in the sense of P. Jolissaint and G. Robertson [22] are purely infinite and minimal (Remark 4.12). In contrast to almost finite groupoids, purely infinite groupoids admit no invariant probability measures. It will be proven that if $G$ is purely infinite and minimal, then the commutator subgroup $D([[G]])$ is simple (Theorem 4.16). We also observe that the topological full group $[[G]]$ for a purely infinite groupoid $G$ contains the free product $\mathbb{Z}_{2} * \mathbb{Z}_{3}$ (Theorem 4.10), and so is not amenable.

In Section 5 we prove that for any purely infinite groupoid $G$, the index map $I:[[G]] \rightarrow$ $H_{1}(G)$ is surjective, where $H_{1}(G)$ stands for the homology group of $G$. The index map $I$ is a homomorphism introduced in $\left[31\right.$, Section 7]. We denote by $[[G]]_{0}$ the kernel of $I$. For almost finite groupoids, the surjectivity of $I$ is already known ([31, Theorem 7.5]). Therefore, if $G$ is almost finite or purely infinite, then the abelianization of $[[G]]$ at least has $H_{1}(G) \cong[[G]] /[[G]]_{0}$ as its quotient.

Section 6 is devoted to the study of the étale groupoids $G$ arising from one-sided irreducible shifts of finite type $(X, \sigma)$. Shifts of finite type (also called topological Markov shifts) form the most prominent class of symbolic dynamical systems. A good introduction to symbolic dynamics can be found in the standard textbook [25] by D. Lind and B. Marcus. From $(X, \sigma)$, we can construct the étale groupoid $G$ by

$$
G=\left\{(x, n, y) \in X \times \mathbb{Z} \times X \mid \exists k, l \in \mathbb{N}, n=k-l, \sigma^{k}(x)=\sigma^{l}(y)\right\} .
$$

By [31, Theorem 4.14], the homology groups $H_{n}(G)$ of $G$ are

$$
H_{n}(G)= \begin{cases}\operatorname{Coker}\left(\mathrm{id}-M^{t}\right) & n=0 \\ \operatorname{Ker}\left(\mathrm{id}-M^{t}\right) & n=1 \\ 0 & n \geq 2\end{cases}
$$

where $M$ is the $k \times k$ matrix with entries in $\mathbb{Z}_{+}$representing $(X, \sigma)$ and is thought of as a homomorphism from $\mathbb{Z}^{k}$ to $\mathbb{Z}^{k}$. Notice that $H_{0}(G)$ is a finitely generated abelian group and $H_{1}(G)$ is isomorphic to the torsion-free part of $H_{0}(G)$. For any non-empty clopen set $Y \subset X$, the reduction $G \mid Y$ of $G$ to $Y$ (see Section 2 for the definition) is again an étale groupoid.

For the topological full group $[[G \mid Y]]$ associated to an irreducible shift of finite type, we have the following.

- $G \mid Y$ is purely infinite and minimal (Theorem 6.1). Hence the commutator subgroup $D([[G \mid Y]])$ is simple by Theorem 4.16 .

- By Theorem 3.10, $[[G \mid Y]]$ (or $[[G \mid Y]]_{0}$ or $D([[G \mid Y]])$ ) is a complete invariant of $G \mid Y$.

- By K. Matsumoto's theorem (Theorem 6.2), for two groupoids $G_{1} \mid Y_{1}$ and $G_{2} \mid Y_{2}$ as above, if there exists an isomorphism $\varphi: H_{0}\left(G_{1}\right) \rightarrow H_{0}\left(G_{2}\right)$ such that $\varphi\left(\left[1_{Y_{1}}\right]_{G_{1}}\right)=$ 
$\left[1_{Y_{2}}\right]_{G_{2}}$ and $\operatorname{det}\left(\mathrm{id}-M_{1}^{t}\right)=\operatorname{det}\left(\mathrm{id}-M_{2}^{t}\right)$, then $G_{1} \mid Y_{1}$ is isomorphic to $G_{2} \mid Y_{2}$ as an étale groupoid.

- $[[G \mid Y]]$ is not amenable (see Theorem 4.10), but has the Haagerup property (Theorem 6.7). This is essentially due to B. Hughes's theorem [21, Theorem 1.1].

- $[[G \mid Y]]$ is of type $\mathrm{F}_{\infty}$ (Theorem [6.21). Thus, $[[G \mid Y]]$ is finitely presented and is of type $\mathrm{FP}_{\infty}$. This is a generalization of K. S. Brown's result [4], which says that the Higman-Thompson group $V_{n, r}$ is of type $\mathrm{F}_{\infty}$ (see also Remark 6.3).

- The abelianization $[[G \mid Y]] / D([[G \mid Y]])$ is isomorphic to $\left(H_{0}(G) \otimes \mathbb{Z}_{2}\right) \oplus H_{1}(G)$ (Corollary 6.24). In particular, $[[G \mid Y]]$ is simple if and only if $H_{0}(G)$ is 2-divisible.

- If $H_{1}(G)=0$, then $[[G \mid Y]]_{0}$ and $D([[G \mid Y]])$ are of type $\mathrm{F}_{\infty}$ (Corollary 6.25 (2)).

- $[[G \mid Y]]_{0}$ and $D([[G \mid Y]])$ are finitely generated (Theorem6.11] and Corollary 6.25 (1)).

- Examples are given in Section 6.7. Among others, the boundary action $\varphi_{k}$ of the free group $\mathbb{F}_{k}$ is discussed (Section 6.7.5). The associated transformation groupoid $G_{\varphi_{k}}$ is naturally isomorphic to an étale groupoid of an irreducible shift of finite type. Hence $\left[\left[G_{\varphi_{k}}\right]\right]$ is of type $\mathrm{F}_{\infty}$ and the commutator subgroup $D\left(\left[\left[G_{\varphi_{k}}\right]\right]\right)$ is simple. The abelianization of $\left[\left[G_{\varphi_{k}}\right]\right]$ is $\mathbb{Z}^{k} \oplus\left(\mathbb{Z}_{2}\right)^{k}$ when $k$ is even and is $\mathbb{Z}^{k} \oplus\left(\mathbb{Z}_{2}\right)^{k+1}$ when $k$ is odd. If $k \neq l$, then $D\left(\left[\left[G_{\varphi_{k}}\right]\right]\right)$ is not isomorphic to $D\left(\left[\left[G_{\varphi_{l}}\right]\right]\right)$.

We have to explain some terminologies in group theory used above. For a natural number $n \in \mathbb{N}$, a group $\Gamma$ is said to be of type $\mathrm{F}_{n}$ if it admits a $K(\Gamma, 1)$ with finite $n$-skeleton, and $\Gamma$ is said to be of type $\mathrm{F}_{\infty}$ if it admits a $K(\Gamma, 1)$ with finite $n$-skeleton in all dimensions $n$ (see [15, Section 7.2]). A group is of type $\mathrm{F}_{1}$ if and only if it is finitely generated, and a group is of type $\mathrm{F}_{2}$ if and only if it is finitely presented ([15, Proposition 7.2.1]). The properties $\mathrm{F}_{n}$ and $\mathrm{F}_{\infty}$ are called topological finiteness properties of $\Gamma$. A group $\Gamma$ is said to be of type $\mathrm{FP}_{n}$ (resp. $\mathrm{FP}_{\infty}$ ) if the trivial $\mathbb{Z} \Gamma$-module $\mathbb{Z}$ admits a projective resolution which is finitely generated in dimensions not greater than $n$ (resp. in all dimensions), cf. [15, Section 8.2]. The properties $\mathrm{FP}_{n}$ and $\mathrm{FP}_{\infty}$ are called homological finiteness properties of $\Gamma$. It is clear that $\mathrm{F}_{n}$ implies $\mathrm{FP}_{n}$. It is known that $\Gamma$ is of type $\mathrm{F}_{\infty}$ if and only if it is of type $\mathrm{FP}_{\infty}$ and is finitely presented (see the proof of [2, Theorem VIII.7.1]).

We would like to mention various known results for the topological full group $\left[\left[G_{\varphi}\right]\right]$ of a minimal action $\varphi: \mathbb{Z} \curvearrowright X$ on a Cantor set $X$. While this is not directly relevant to the present article, the reader may find interesting similarities to the case of shifts of finite type. By [17, Corollary 4.4] and [1, Theorem 5.13], $\left[\left[G_{\varphi}\right]\right]$ (or $\left[\left[G_{\varphi}\right]\right]_{0}$ or $D\left(\left[\left[G_{\varphi}\right]\right]\right)$ ) is a complete invariant of $G_{\varphi}$ (see also Theorem 3.9). By Theorem 4.7, the commutator subgroup $D\left(\left[\left[G_{\varphi}\right]\right]\right)$ is simple (this was first proved in [30, Theorem 4.9]). The abelianization $\left[\left[G_{\varphi}\right]\right] / D\left(\left[\left[G_{\varphi}\right]\right]\right)$ is known to be isomorphic to $\left(H_{0}\left(G_{\varphi}\right) \otimes \mathbb{Z}_{2}\right) \oplus \mathbb{Z}$ (see [30, Theorem 4.8]). Note that $H_{1}\left(G_{\varphi}\right)$ is always $\mathbb{Z}$ in this setting. By [30, Theorem 5.4], $D\left(\left[\left[G_{\varphi}\right]\right]\right)$ is finitely generated if and only if $\varphi$ is expansive (i.e. $\varphi$ is conjugate to a two-sided minimal subshift). However, $\left[\left[G_{\varphi}\right]\right],\left[\left[G_{\varphi}\right]\right]_{0}$ and $D\left(\left[\left[G_{\varphi}\right]\right]\right)$ are never finitely presented ([30, Theorem 5.7, Corollary 5.8]). When $D\left(\left[\left[G_{\varphi}\right]\right]\right)$ is finitely generated, it is known that $D\left(\left[\left[G_{\varphi}\right]\right]\right)$ has exponential growth ([32, Corollary 2.5]). R. Grigorchuk and K. Medynets [18] proved that $\left[\left[G_{\varphi}\right]\right]$ is locally embeddable into finite groups, and conjectured that $\left[\left[G_{\varphi}\right]\right]$ is amenable. 
K. Juschenko and N. Monod [23] recently confirmed this conjecture. This provided the first examples of finitely generated simple amenable infinite groups.

\section{Preliminaries}

The cardinality of a set $A$ is written \# $A$ and the characteristic function of $A$ is written $1_{A}$. The finite cyclic group of order $n$ is denoted by $\mathbb{Z}_{n}$. We say that a subset of a topological space is clopen if it is both closed and open. A topological space is said to be totally disconnected if its topology is generated by clopen subsets. By a Cantor set, we mean a compact, metrizable, totally disconnected space with no isolated points. It is known that any two such spaces are homeomorphic. The homeomorphism group of a topological space $X$ is written $\operatorname{Homeo}(X)$. For $\alpha \in \operatorname{Homeo}(X)$, we let $\operatorname{supp}(\alpha)$ denote the closure of $\{x \in X \mid \alpha(x) \neq x\}$. The commutator of $\alpha, \beta \in \operatorname{Homeo}(X)$ is $[\alpha, \beta]=\alpha \beta \alpha^{-1} \beta^{-1}$. The commutator subgroup of a group $\Gamma$ is denoted by $D(\Gamma)$.

In this article, by an étale groupoid we mean a second countable locally compact Hausdorff groupoid such that the range map is a local homeomorphism. We refer the reader to [37, 38] for background material on étale groupoids. For an étale groupoid $G$, we let $G^{(0)}$ denote the unit space and let $s$ and $r$ denote the source and range maps. For $x \in G^{(0)}, G(x)=r(G x)$ is called the $G$-orbit of $x$. When every $G$-orbit is dense in $G^{(0)}, G$ is said to be minimal. For an open subset $Y \subset G^{(0)}$, the reduction of $G$ to $Y$ is $r^{-1}(Y) \cap s^{-1}(Y)$ and denoted by $G \mid Y$. The reduction $G \mid Y$ is an étale subgroupoid of $G$ in an obvious way. The isotropy bundle is $G^{\prime}=\{g \in G \mid r(g)=s(g)\}$. We say that $G$ is principal if $G^{\prime}=G^{(0)}$. When the interior of $G^{\prime}$ is $G^{(0)}$, we say that $G$ is essentially principal. A subset $U \subset G$ is called a $G$-set if $r|U, s| U$ are injective. When $U, V$ are $G$-sets,

$$
U^{-1}=\left\{g \in G \mid g^{-1} \in U\right\}
$$

and

$$
U V=\left\{g g^{\prime} \in G \mid g \in U, g^{\prime} \in V, s(g)=r\left(g^{\prime}\right)\right\}
$$

are also $G$-sets. For an open $G$-set $U$, we let $\pi_{U}$ denote the homeomorphism $r \circ(s \mid U)^{-1}$ from $s(U)$ to $r(U)$. For any compact open $G$-set $U, G \mid r(U)$ is naturally isomorphic to $G \mid s(U)$. A probability measure $\mu$ on $G^{(0)}$ is said to be $G$-invariant if $\mu(r(U))=\mu(s(U))$ holds for every open $G$-set $U$. The set of all $G$-invariant measures is denoted by $M(G)$. For an étale groupoid $G$, we denote the reduced groupoid $C^{*}$-algebra of $G$ by $C_{r}^{*}(G)$ and identify $C_{0}\left(G^{(0)}\right)$ with a subalgebra of $C_{r}^{*}(G)$. J. Renault's theorem [38, Theorem 4.11] tells us that two essentially principal étale groupoids $G_{1}$ and $G_{2}$ are isomorphic if and only if there exists an isomorphism $\varphi: C_{r}^{*}\left(G_{1}\right) \rightarrow C_{r}^{*}\left(G_{2}\right)$ such that $\varphi\left(C_{0}\left(G_{1}^{(0)}\right)\right)=C_{0}\left(G_{2}^{(0)}\right)$ (see also [31, Theorem 5.1]).

We would like to recall the notion of topological full groups for étale groupoids.

Definition 2.1 ([31, Definition 2.3]). Let $G$ be an essentially principal étale groupoid whose unit space $G^{(0)}$ is compact.

(1) The set of all $\alpha \in \operatorname{Homeo}\left(G^{(0)}\right)$ such that for every $x \in G^{(0)}$ there exists $g \in G$ satisfying $r(g)=x$ and $s(g)=\alpha(x)$ is called the full group of $G$ and denoted by $[G]$. 
(2) The set of all $\alpha \in \operatorname{Homeo}\left(G^{(0)}\right)$ for which there exists a compact open $G$-set $U$ satisfying $\alpha=\pi_{U}$ is called the topological full group of $G$ and denoted by $[[G]]$.

Obviously $[G]$ is a subgroup of $\operatorname{Homeo}\left(G^{(0)}\right)$ and $[[G]]$ is a subgroup of $[G]$.

For $\alpha \in[[G]]$ the compact open $G$-set $U$ as above uniquely exists, because $G$ is essentially principal. Obviously $[[G]]$ is a subgroup of $\operatorname{Homeo}\left(G^{(0)}\right)$. Since $G$ is second countable, it has countably many compact open subsets, and so $[[G]]$ is at most countable. By [31, Proposition 5.6], we have the following short exact sequence:

$$
1 \longrightarrow U\left(C\left(G^{(0)}\right)\right) \longrightarrow N\left(C\left(G^{(0)}\right), C_{r}^{*}(G)\right) \stackrel{\sigma}{\longrightarrow}[[G]] \longrightarrow 1,
$$

where $U\left(C\left(G^{(0)}\right)\right)$ denotes the group of unitaries in $C\left(G^{(0)}\right)$ and $N\left(C\left(G^{(0)}\right), C_{r}^{*}(G)\right)$ denotes the group of unitaries in $C_{r}^{*}(G)$ which normalize $C\left(G^{(0)}\right)$. In addition, the homomorphism $\sigma$ has a right inverse.

Lemma 2.2. Let $G$ be an essentially principal étale groupoid whose unit space $G^{(0)}$ is compact. Then $\operatorname{supp}(\alpha)$ is clopen for any $\alpha \in[[G]]$.

Proof. There exists a compact open $G$-set $U$ such that $\alpha=\pi_{U}$. The map $s \mid U: U \rightarrow G^{(0)}$ is a homeomorphism. It suffices to show that $\operatorname{supp}(\alpha)=s\left(U \backslash G^{(0)}\right)$. Since $G^{(0)}$ is open and $s(g)=g=r(g)$ for $g \in G^{(0)}$, it is evident that $\operatorname{supp}(\alpha)$ is contained in $s\left(U \backslash G^{(0)}\right)$. It is also clear that $s\left(U \backslash G^{\prime}\right)$ is contained in $\operatorname{supp}(\alpha)$. Therefore $s\left(U \backslash G^{(0)}\right)$ is contained in $\operatorname{supp}(\alpha)$, because $G$ is essentially principal.

In [31, Section 7], we introduced the index map $I:[[G]] \rightarrow H_{1}(G)$, where $H_{1}(G)$ is the homology group of $G$ (see [31, Section 3]). For $\alpha \in[[G]]$, let $U \subset G$ be the compact open $G$-set such that $\alpha=\pi_{U}$. Then the characteristic function $1_{U} \in C_{c}(G, \mathbb{Z})$ is a cycle, and $I(\alpha)$ is the equivalence class of $1_{U}$ in $H_{1}(G)$. The index map $I$ is a homomorphism. We let $[[G]]_{0}$ denote the kernel of the index map $I$. Evidently $D([[G]])$ is contained in $[[G]]_{0}$. The main objective of the present paper is to study various properties of the groups $D([[G]]) \subset[[G]]_{0} \subset[[G]]$.

\section{A spatial realization theorem}

In this section, we will prove that any isomorphism between (certain normal subgroups of) topological full groups is realized by a homeomorphism of the underlying spaces (Theorem 3.5. Proposition 3.6). In particular, these various groups (as abstract groups) are complete invariants for étale groupoids (Theorem 3.10). Our proof of Theorem 3.5) is essentially the same as that of [14, Theorem 384D], and a similar argument can be found in [1, Section $5]$.

Definition 3.1. Let $X$ be a Cantor set and let $\Gamma \subset \operatorname{Homeo}(X)$ be a subgroup. We say that $\Gamma$ is of class $F$ if the following conditions are satisfied.

(F0) For any $\alpha \in \Gamma$ satisfying $\alpha^{2}=1, \operatorname{supp}(\alpha)$ is clopen.

(F1) For any $x \in X$ and any clopen neighborhood $A \subset X$ of $x$, there exists $\alpha \in \Gamma \backslash\{1\}$ such that $x \in \operatorname{supp}(\alpha), \operatorname{supp}(\alpha) \subset A$ and $\alpha^{2}=1$. 
(F2) For any $\alpha \in \Gamma \backslash\{1\}$ satisfying $\alpha^{2}=1$ and any non-empty clopen set $A \subset \operatorname{supp}(\alpha)$, there exists $\beta \in \Gamma \backslash\{1\}$ such that $\operatorname{supp}(\beta) \subset A \cup \alpha(A)$ and $\beta(x)=\alpha(x)$ for every $x \in \operatorname{supp}(\beta)$.

(F3) For any non-empty clopen set $A \subset X$, there exists $\alpha \in \Gamma$ such that $\operatorname{supp}(\alpha) \subset A$ and $\alpha^{2} \neq 1$.

For $A \subset X$, we write

$$
\Gamma(A)=\{\alpha \in \Gamma \mid \operatorname{supp}(\alpha) \subset A\} .
$$

A closed set $A$ is said to be regular if it is equal to the closure of its interior.

Lemma 3.2. Let $X$ be a Cantor set and let $\Gamma \subset \operatorname{Homeo}(X)$ be a subgroup of class $F$. For two regular closed sets $A, B \subset X, \Gamma(A) \subset \Gamma(B)$ if and only if $A \subset B$.

Proof. It is clear that $A \subset B$ implies $\Gamma(A) \subset \Gamma(B)$. Suppose that $A \backslash B$ is not empty. Since $A$ is regular, $A \backslash B$ has non-empty interior. It follows from (F1) (or (F3)) that there exists $\alpha \in \Gamma \backslash\{1\}$ such that $\operatorname{supp}(\alpha) \subset A \backslash B$. Hence $\Gamma(A)$ is not contained in $\Gamma(B)$.

Let $X$ be a Cantor set and let $\Gamma \subset \operatorname{Homeo}(X)$ be a subgroup of class F. For $\tau \in \Gamma \backslash\{1\}$ satisfying $\tau^{2}=1$, we define $C_{\tau}, U_{\tau}, S_{\tau}$ and $W_{\tau}$ as follows. First, let $C_{\tau}$ be the centralizer of $\tau$, that is,

$$
C_{\tau}=\{\alpha \in \Gamma \mid[\alpha, \tau]=1\}
$$

Define a subset $U_{\tau} \subset C_{\tau}$ by

$$
U_{\tau}=\left\{\sigma \in C_{\tau} \mid \sigma^{2}=1, \quad\left[\sigma, \alpha \sigma \alpha^{-1}\right]=1 \quad \forall \alpha \in C_{\tau}\right\} .
$$

We define $S_{\tau}$ by

$$
S_{\tau}=\left\{\alpha^{2} \in \Gamma \mid \alpha \in \Gamma,[\alpha, \sigma]=1 \quad \forall \sigma \in U_{\tau}\right\} .
$$

Then, we let $W_{\tau}$ be the centralizer of $S_{\tau}$, that is,

$$
W_{\tau}=\left\{\alpha \in \Gamma \mid[\alpha, \beta]=1 \quad \forall \beta \in S_{\tau}\right\} .
$$

For these subsets, we can prove the following.

Lemma 3.3. Let the notation be as above.

(1) For any $\sigma \in U_{\tau}$ and $x \notin \operatorname{supp}(\tau)$, we have $\sigma(x)=x$.

(2) For any non-empty clopen set $A \subset \operatorname{supp}(\tau)$, there exists $\sigma \in U_{\tau}$ such that $\operatorname{supp}(\sigma) \subset$ $A \cup \tau(A)$ and $\sigma(x)=\tau(x)$ for every $x \in \operatorname{supp}(\sigma)$.

(3) For any non-empty clopen set $A$ such that $A \cap \operatorname{supp}(\tau)=\emptyset$, there exists $\alpha \in S_{\tau} \backslash\{1\}$ such that $\operatorname{supp}(\alpha) \subset A$.

(4) For any $\alpha \in S_{\tau}$ and $x \in \operatorname{supp}(\tau), \alpha(x)=x$.

(5) We have $W_{\tau}=\Gamma(\operatorname{supp}(\tau))$. 
Proof. (1) Let $\sigma \in U_{\tau}$ and $x \notin \operatorname{supp}(\tau)$. Assume $\sigma(x) \neq x$. There exists a clopen neighborhood $A \subset X$ of $x$ such that $A \cap \sigma(A)=\emptyset$ and $A \cap \operatorname{supp}(\tau)=\emptyset$. By (F3), we can find $\alpha \in \Gamma$ such that $\operatorname{supp}(\alpha) \subset A$ and $\alpha^{2} \neq 1$. Clearly $\alpha$ commutes with $\tau$, and so $\alpha$ is in $C_{\tau}$. There exists $y \in A$ such that $\alpha^{-1}(y), y, \alpha(y)$ are mutually distinct. Then we have

$$
\left(\sigma \alpha \sigma \alpha^{-1}\right)(y)=\alpha^{-1}(y)
$$

and

$$
\left(\alpha \sigma \alpha^{-1} \sigma\right)(y)=\alpha(y)
$$

which contradict $\left[\sigma, \alpha \sigma \alpha^{-1}\right]=1$.

(2) Let $A \subset \operatorname{supp}(\tau)$ be a non-empty clopen set. By (F2), there exists $\sigma \in \Gamma$ such that $\operatorname{supp}(\sigma) \subset A \cup \tau(A)$ and $\sigma(x)=\tau(x)$ for every $x \in \operatorname{supp}(\sigma)$. It suffices to show that $\sigma$ is in $U_{\tau}$. It is easy to see $\sigma \in C_{\tau}$ and $\sigma^{2}=1$. Let $\alpha \in C_{\tau}$. Then, for any $x \in \operatorname{supp}\left(\alpha \sigma \alpha^{-1}\right)=\alpha(\operatorname{supp}(\sigma))$, one has $\left(\alpha \sigma \alpha^{-1}\right)(x)=\left(\alpha \tau \alpha^{-1}\right)(x)=\tau(x)$. It follows that $\sigma$ commutes with $\alpha \sigma \alpha^{-1}$, and hence $\sigma$ belongs to $U_{\tau}$.

(3) Let $A$ be a non-empty clopen set such that $A \cap \operatorname{supp}(\tau)=\emptyset$. By (F3), there exists $\alpha \in \Gamma$ such that $\operatorname{supp}(\alpha) \subset A$ and $\alpha^{2} \neq 1$. It follows from (1) that $\alpha$ commutes with any elements in $U_{\tau}$. Therefore $\alpha^{2}$ is in $S_{\tau} \backslash\{1\}$.

(4) Suppose that $\alpha \in \Gamma$ commutes with any elements in $U_{\tau}$. It suffices to show that if $x \neq \tau(x)$, then $\alpha(x)$ is equal to either $x$ or $\tau(x)$. Assume that $x, \tau(x), \alpha(x)$ are mutually distinct. There exists a clopen neighborhood $A \subset \operatorname{supp}(\tau)$ of $x$ such that $A, \tau(A), \alpha(A)$ are mutually disjoint. By (2), we can find $\sigma \in U_{\tau}$ such that $\operatorname{supp}(\sigma) \subset A \cup \tau(A)$ and $\sigma(y)=\tau(y)$ for every $y \in \operatorname{supp}(\sigma)$. Then $(\sigma \alpha)(y)=\alpha(y)$ is not equal to $(\alpha \sigma)(y)$ for $y \in \operatorname{supp}(\sigma)$, which is a contradiction.

(5) From (4), we easily obtain $\Gamma(\operatorname{supp}(\tau)) \subset W_{\tau}$. Let us show $W_{\tau} \subset \Gamma(\operatorname{supp}(\tau))$. Let $\alpha \in W_{\tau}$ and $x \notin \operatorname{supp}(\tau)$. Assume $\alpha(x) \neq x$. There exists a non-empty clopen neighborhood $A$ of $x$ such that $A \cap \operatorname{supp}(\tau)=\emptyset$ and $A \cap \alpha(A)=\emptyset$. By (3), there exists $\beta \in S_{\tau} \backslash\{1\}$ such that $\operatorname{supp}(\beta) \subset A$. Then $\beta$ is not equal to $\alpha \beta \alpha^{-1}$, which is a contradiction. Therefore $\alpha(x)=x$, and whence $\alpha \in \Gamma(\operatorname{supp}(\tau))$.

Lemma 3.4. For $i=1,2$, let $X_{i}$ be a Cantor set and let $\Gamma_{i} \subset \operatorname{Homeo}\left(X_{i}\right)$ be a subgroup of class F. Suppose that $\Phi: \Gamma_{1} \rightarrow \Gamma_{2}$ is an isomorphism. Let $\tau, \sigma \in \Gamma_{1}$ be such that $\tau^{2}=\sigma^{2}=1$.

(1) We have $\operatorname{supp}(\tau) \subset \operatorname{supp}(\sigma)$ if and only if $\operatorname{supp}(\Phi(\tau)) \subset \operatorname{supp}(\Phi(\sigma))$.

(2) We have $\operatorname{supp}(\tau) \cap \operatorname{supp}(\sigma)=\emptyset$ if and only if $\operatorname{supp}(\Phi(\tau)) \cap \operatorname{supp}(\Phi(\sigma))=\emptyset$.

Proof. First, we note that $\Phi\left(W_{\tau}\right)$ equals $W_{\Phi(\tau)}$, because the definition of $W_{\tau}$ is purely algebraic.

(1) From Lemma 3.2 and Lemma 3.3 (5), we get

$$
\operatorname{supp}(\tau) \subset \operatorname{supp}(\sigma) \Rightarrow \Gamma_{1}(\operatorname{supp}(\tau)) \subset \Gamma_{1}(\operatorname{supp}(\sigma)) \Rightarrow W_{\tau} \subset W_{\sigma} \Rightarrow \Phi\left(W_{\tau}\right) \subset \Phi\left(W_{\sigma}\right),
$$

which means $W_{\Phi(\tau)} \subset W_{\Phi(\sigma)}$. Using Lemma 3.2 and Lemma 3.3 (5) again, we have

$$
W_{\Phi(\tau)} \subset W_{\Phi(\sigma)} \Rightarrow \Gamma_{2}(\operatorname{supp}(\Phi(\tau))) \subset \Gamma_{2}(\operatorname{supp}(\Phi(\sigma))) \Rightarrow \operatorname{supp}(\Phi(\tau)) \subset \operatorname{supp}(\Phi(\sigma)) .
$$


(2) Suppose that $\operatorname{supp}(\tau) \cap \operatorname{supp}(\sigma)$ is not empty. By (F0), $\operatorname{supp}(\tau) \cap \operatorname{supp}(\sigma)$ has non-empty interior. It follows from (F1) that there exists $\rho \in \Gamma \backslash\{1\}$ such that $\rho^{2}=1$ and $\operatorname{supp}(\rho) \subset \operatorname{supp}(\tau) \cap \operatorname{supp}(\sigma)$. From $(1)$, we get $\operatorname{supp}(\Phi(\rho)) \subset \operatorname{supp}(\Phi(\tau)) \cap \operatorname{supp}(\Phi(\sigma))$. Thus $\operatorname{supp}(\Phi(\tau)) \cap \operatorname{supp}(\Phi(\sigma))$ is not empty.

The following theorem is a generalization of [17, Theorem 4.2] and [29, Theorem 7.2]. K. Medynets 33 also obtained a similar result for (topological) full groups of transformation groupoids (see [33, Remark 3]).

Theorem 3.5. For $i=1,2$, let $X_{i}$ be a Cantor set and let $\Gamma_{i} \subset \operatorname{Homeo}\left(X_{i}\right)$ be a subgroup of class $F$. Suppose that $\Phi: \Gamma_{1} \rightarrow \Gamma_{2}$ is an isomorphism. Then there exists a homeomorphism $\varphi: X_{1} \rightarrow X_{2}$ such that $\Phi(\alpha)=\varphi \circ \alpha \circ \varphi^{-1}$ for all $\alpha \in \Gamma_{1}$.

Proof. For $x \in X_{i}$, we let

$$
T(x)=\left\{\tau \in \Gamma_{i} \mid x \in \operatorname{supp}(\tau), \tau^{2}=1\right\} .
$$

We first claim that for every $x \in X_{1}$, the set

$$
P(x)=\bigcap_{\tau \in T(x)} \operatorname{supp}(\Phi(\tau)) \subset X_{2}
$$

is a singleton. Let $\tau_{1}, \tau_{2}, \ldots, \tau_{n} \in T(x)$. By $(\mathrm{F} 0), \operatorname{supp}\left(\tau_{i}\right)$ is clopen, and so there exists a clopen neighborhood $A$ of $x$ such that $A \subset \operatorname{supp}\left(\tau_{i}\right)$ for all $i=1,2, \ldots, n$. By $(\mathrm{F} 1)$, we can find $\tau \in T(x) \cap \Gamma_{1}(A)$. It follows from the lemma above that $\operatorname{supp}(\Phi(\tau))$ is contained in $\operatorname{supp}\left(\Phi\left(\tau_{i}\right)\right)$ for every $i=1,2, \ldots, n$. Hence the sets $\operatorname{supp}\left(\Phi\left(\tau_{i}\right)\right)$ have nonempty intersection. From the compactness of $X_{2}$, we can conclude that $P(x)$ is not empty. Assume that $P(x)$ contains two distinct points $y, y^{\prime} \in X_{2}$. By (F1), there exists $\sigma \in T(y)$ such that $y^{\prime} \notin \operatorname{supp}(\sigma)$. If $x$ does not belong to $\operatorname{supp}\left(\Phi^{-1}(\sigma)\right)$, then by (F1) there exists $\tau \in T(x)$ such that $\operatorname{supp}(\tau) \cap \operatorname{supp}\left(\Phi^{-1}(\sigma)\right)=\emptyset$. By the lemma above, one has $\operatorname{supp}(\Phi(\tau)) \cap \operatorname{supp}(\sigma)=\emptyset$. Since $y$ is in $P(x)$ and $P(x)$ is contained in $\operatorname{supp}(\Phi(\tau))$, $\operatorname{supp}(\Phi(\tau))$ intersects with $\operatorname{supp}(\sigma)$, which is a contradiction. Hence $x$ is in $\operatorname{supp}\left(\Phi^{-1}(\sigma)\right)$, that is, $\Phi^{-1}(\sigma)$ is in $T(x)$. Then $P(x)$ is contained in $\operatorname{supp}(\sigma)$, and so $y^{\prime}$ is not in $P(x)$, which is again a contradiction.

Now we can define a map $\varphi: X_{1} \rightarrow X_{2}$ by $\{\varphi(x)\}=P(x)$. It is easy to see that $\varphi(\operatorname{supp}(\tau)) \subset \operatorname{supp}(\Phi(\tau))$ holds for any $\tau \in \Gamma_{1}$ such that $\tau^{2}=1$. We would like to show that $\varphi$ is continuous. Let $A$ be an open neighborhood of $\varphi(x)$. By the definition of $P(x)$, there exist $\tau_{1}, \tau_{2}, \ldots, \tau_{n} \in T(x)$ such that the intersection of the sets $\operatorname{supp}\left(\Phi\left(\tau_{i}\right)\right)$ is contained in $A$. In the same way as above, using (F0) and (F1), we can find $\tau \in T(x)$ satisfying $\operatorname{supp}(\tau) \subset \operatorname{supp}\left(\tau_{i}\right)$. Since $\operatorname{supp}(\tau)$ is a clopen neighborhood of $x$ by (F0) and

$$
\varphi(\operatorname{supp}(\tau)) \subset \operatorname{supp}(\Phi(\tau)) \subset \bigcap_{i=1}^{n} \operatorname{supp}\left(\Phi\left(\tau_{i}\right)\right) \subset A,
$$

$\varphi$ is continuous at $x$.

In exactly the same way, we can construct a continuous map $\varphi^{\prime}: X_{2} \rightarrow X_{1}$ such that

$$
\left\{\varphi^{\prime}(y)\right\}=\bigcap_{\sigma \in T(y)} \operatorname{supp}\left(\Phi^{-1}(\sigma)\right) \quad \forall y \in X_{2}
$$


For any $x \in X_{1}$, by (F1), we have

$$
\left\{\varphi^{\prime}(\varphi(x))\right\}=\bigcap_{\sigma \in T(\varphi(x))} \operatorname{supp}\left(\Phi^{-1}(\sigma)\right) \subset \bigcap_{\tau \in T(x)} \operatorname{supp}\left(\Phi^{-1}(\Phi(\tau))\right)=\{x\}
$$

which means $\varphi^{\prime} \circ \varphi=$ id. Similarly $\varphi \circ \varphi^{\prime}=$ id. Thus $\varphi$ is a homeomorphism and $\varphi^{-1}=\varphi^{\prime}$.

Let $\alpha \in \Gamma_{1}$. We would like to show $\Phi(\alpha)=\varphi \circ \alpha \circ \varphi^{-1}$. For any $\tau \in \Gamma_{1}$ satisfying $\tau^{2}=1$, one has

$$
(\Phi(\alpha) \circ \varphi)(\operatorname{supp}(\tau))=\Phi(\alpha)\left(\operatorname{supp}(\Phi(\tau))=\operatorname{supp}\left(\Phi\left(\alpha \tau \alpha^{-1}\right)\right)\right.
$$

and

$$
(\varphi \circ \alpha)(\operatorname{supp}(\tau))=\varphi\left(\operatorname{supp}\left(\alpha \tau \alpha^{-1}\right)\right)=\operatorname{supp}\left(\Phi\left(\alpha \tau \alpha^{-1}\right)\right) .
$$

Since $\{x\}=\bigcap_{\tau \in T(x)} \operatorname{supp}(\tau)$ for any $x \in X_{1}$, we can conclude $\Phi(\alpha) \circ \varphi=\varphi \circ \alpha$.

Proposition 3.6. Let $G$ be an essentially principal étale groupoid whose unit space is a Cantor set. Suppose that $G$ is minimal. Then any subgroup $\Gamma \subset[[G]]$ containing $D([[G]])$ is of class $F$.

Proof. Let $\Gamma \subset[[G]]$ be a subgroup such that $D([[G]]) \subset \Gamma$. We verify the conditions of Definition 3.1 for $\Gamma$. Condition (F0) immediately follows from Lemma 2.2 .

To show (F1), let $A \subset G^{(0)}$ be a clopen neighborhood of $x \in G^{(0)}$. Since $G$ is minimal, there exists a compact open $G$-set $U \subset G$ such that $x \in s(U), s(U) \cup r(U) \subset A$ and $s(U) \cap r(U)=\emptyset$. Define $\alpha \in[[G]]$ by

$$
\alpha(x)= \begin{cases}\pi_{U}(x) & x \in s(U) \\ \pi_{U}^{-1}(x) & x \in r(U) \\ x & \text { otherwise }\end{cases}
$$

We can also find a compact open $G$-set $V \subset G$ such that $x \in s(V), s(V) \cup r(V) \subset s(U)$ and $s(V) \cap r(V)=\emptyset$, because $G$ is minimal. Define $\beta \in[[G]]$ by

$$
\beta(x)= \begin{cases}\pi_{V}(x) & x \in s(V) \\ \pi_{V}^{-1}(x) & x \in r(V) \\ x & \text { otherwise. }\end{cases}
$$

Then $[\alpha, \beta]=\alpha \beta \alpha \beta \in D[[G]]$ satisfies the requirement.

In order to prove (F2), we take $\alpha \in[[G]] \backslash\{1\}$ satisfying $\alpha^{2}=1$ and a non-empty clopen set $A \subset \operatorname{supp}(\alpha)$. Since $G$ is minimal, we can find a non-empty compact open $G$-set $V$ such that $s(V) \cup r(V) \subset A$ and $s(V), r(V), \alpha(s(V)), \alpha(r(V))$ are mutually disjoint. Using $V$, we define $\beta \in[[G]]$ as above. Define $\tilde{\alpha}, \tilde{\beta} \in[[G]]$ by

$$
\tilde{\alpha}(x)= \begin{cases}\alpha(x) & x \in s(V) \cup \alpha(s(V)) \\ x & \text { otherwise }\end{cases}
$$

and $\tilde{\beta}=[\alpha, \beta]=\alpha \beta \alpha \beta$. Then $[\tilde{\alpha}, \tilde{\beta}] \in D([[G]])$ satisfies the requirement. 
Let us consider (F3). Since $G$ is minimal, there exist non-empty compact open $G$-sets $U_{1}, U_{2}$ satisfying $s\left(U_{i}\right) \cup r\left(U_{i}\right) \subset A, s\left(U_{i}\right) \cap r\left(U_{i}\right)=\emptyset, r\left(U_{1}\right) \cap s\left(U_{2}\right)=\emptyset$ and $s\left(U_{1}\right)=r\left(U_{2}\right)$. For $i=1,2$, we define $\alpha_{i} \in[[G]]$ by

$$
\alpha_{i}(x)= \begin{cases}\pi_{U_{i}}(x) & x \in s\left(U_{i}\right) \\ \pi_{U_{i}}^{-1}(x) & x \in r\left(U_{i}\right) \\ x & \text { otherwise }\end{cases}
$$

Then $\left[\alpha_{1}, \alpha_{2}\right] \in D([[G]])$ is of order three.

Lemma 3.7. Let $G$ be an essentially principal étale groupoid whose unit space is a Cantor set. Suppose that every $G$-orbit contains at least three points. For any $g \in G$, there exists a compact open $G$-set $U$ such that $\pi_{U} \in D([[G]])$ and $g \in U$.

Proof. First, let us assume $s(g) \neq r(g)$. Since the $G$-orbit of $s(g)$ contains at least three points, there exist compact open $G$-sets $U$ and $V$ such that the following hold.

- $s(U)=r(V)$.

- $r(U), s(U)$ and $s(V)$ are mutually disjoint.

- $g \in U$.

Then

$$
W=U \cup V \cup(U V)^{-1} \cup\left(G^{(0)} \backslash(r(U) \cup s(U) \cup s(V))\right)
$$

is a desired compact open $G$-set. Next, assume $s(g)=r(g)$. We can find $g_{1}, g_{2} \in G$ such that $s\left(g_{i}\right) \neq r\left(g_{i}\right)$ for $i=1,2$ and $g=g_{1} g_{2}$. By the argument above, the proof is complete.

Proposition 3.8. For $i=1,2$, let $G_{i}$ be an essentially principal étale groupoid whose unit space is a Cantor set. Suppose that every $G_{i}$-orbit contains at least three points. For each $i=1,2$, let $\Gamma_{i}$ be a subgroup of $\left[\left[G_{i}\right]\right]$ such that $D\left(\left[\left[G_{i}\right]\right]\right) \subset \Gamma_{i}$. If there exists a homeomorphism $\varphi: G_{1}^{(0)} \rightarrow G_{2}^{(0)}$ such that $\alpha \mapsto \varphi \circ \alpha \circ \varphi^{-1}$ gives an isomorphism from $\Gamma_{1}$ to $\Gamma_{2}$, then $G_{1}$ and $G_{2}$ are isomorphic as étale groupoids.

Proof. For $g \in G_{1}$, by the lemma above, we can find a compact open $G_{1}$-set $U$ such that $\pi_{U} \in D\left(\left[\left[G_{1}\right]\right]\right) \subset \Gamma_{1}$ and $g \in U$. By assumption, $\varphi \circ \pi_{U} \circ \varphi^{-1}$ belongs to $\Gamma_{2}$. Thus, there exists a compact open $G_{2}$-set $V$ such that $\pi_{V}=\varphi \circ \pi_{U} \circ \varphi^{-1}$. It follows that there exists $g^{\prime} \in G_{2}$ such that $g^{\prime} \in V$ and $s\left(g^{\prime}\right)=\varphi(s(g))$. It is routine to check that the map $g \mapsto g^{\prime}$ is a well-defined isomorphism from $G_{1}$ to $G_{2}$.

The following theorem is a generalization of several results obtained in [17, Section 4] and [29, Corollary 7.3].

Theorem 3.9. For $i=1,2$, let $G_{i}$ be an essentially principal étale groupoid whose unit space is a Cantor set. Suppose that $G_{i}$ is minimal. For each $i=1,2$, let $\Gamma_{i}$ be a subgroup of $\left[\left[G_{i}\right]\right]$ such that $D\left(\left[\left[G_{i}\right]\right]\right) \subset \Gamma_{i}$. If $\Gamma_{1}$ and $\Gamma_{2}$ are isomorphic as discrete groups, then $G_{1}$ and $G_{2}$ are isomorphic as étale groupoids. 
Proof. This readily follows from Theorem 3.5, Proposition 3.6 and Proposition 3.8 .

As an immediate consequence of the theorem above, we obtain the following.

Theorem 3.10. For $i=1,2$, let $G_{i}$ be an essentially principal étale groupoid whose unit space is a Cantor set and suppose that $G_{i}$ is minimal. The following conditions are equivalent.

(1) $G_{1}$ and $G_{2}$ are isomorphic as étale groupoids.

(2) $\left[\left[G_{1}\right]\right]$ and $\left[\left[G_{2}\right]\right]$ are isomorphic as discrete groups.

(3) $\left[\left[G_{1}\right]\right]_{0}$ and $\left[\left[G_{2}\right]\right]_{0}$ are isomorphic as discrete groups.

(4) $D\left(\left[\left[G_{1}\right]\right]\right)$ and $D\left(\left[\left[G_{2}\right]\right]\right)$ are isomorphic as discrete groups.

\section{Simplicity of commutator subgroups}

\subsection{Almost finite groupoids}

Throughout this subsection, we let $G$ be an essentially principal étale groupoid whose unit space is a Cantor set. In this subsection, we would like to show that the commutator subgroup $D([[G]])$ of the topological full group $[[G]]$ is simple when $G$ is almost finite and minimal (Theorem 4.7). This is a generalization of [30, Theorem 4.9] and [1, Theorem 3.4]. Let us recall the definition of almost finite groupoids.

Definition 4.1 ([31, Definition 6.2]). Let $G$ be an étale groupoid whose unit space is a Cantor set.

(1) We say that $K \subset G$ is an elementary subgroupoid if $K$ is a compact open principal subgroupoid of $G$ such that $K^{(0)}=G^{(0)}$.

(2) We say that $G$ is almost finite if for any compact subset $C \subset G$ and $\varepsilon>0$ there exists an elementary subgroupoid $K \subset G$ such that

$$
\frac{\#(C K x \backslash K x)}{\#(K x)}<\varepsilon
$$

for all $x \in G^{(0)}$. We also remark that \# $(K(x))$ equals \# $(K x)$, because $K$ is principal.

In [31, Lemma 6.3], it was shown that when $\varphi: \mathbb{Z}^{N} \curvearrowright X$ is a free action of $\mathbb{Z}^{N}$ on a Cantor set $X$, the transformation groupoid $G_{\varphi}$ is almost finite (see [31, Definition 2.1] for the definition of $G_{\varphi}$ ).

A homeomorphism $\alpha$ of a Cantor set $X$ is said to be elementary if it is of finite order and $\left\{x \in X \mid \alpha^{k}(x)=x\right\}$ is clopen for every $k \in \mathbb{N}$ ([31, Definition 7.6 (1)]).

Lemma 4.2. Suppose that $G$ is almost finite. For any $\varepsilon>0$ and $\alpha \in[[G]]$, there exists an elementary homeomorphism $\alpha_{0} \in[[G]]$ such that $\mu\left(\operatorname{supp}\left(\alpha_{0} \alpha\right)\right) \leq \varepsilon$ for any $\mu \in M(G)$.

Proof. This statement is almost the same as [31, Lemma 7.10] and is shown by a small modification of its proof. 
Lemma 4.3. Suppose that every G-orbit is infinite. For any elementary homeomorphism $\alpha \in[[G]]$ and $\varepsilon>0$, there exist elementary homeomorphisms $\alpha_{1}, \alpha_{2}, \ldots, \alpha_{n} \in[[G]]$ such that $\alpha=\alpha_{1} \alpha_{2} \ldots \alpha_{n}$ and $\mu\left(\operatorname{supp}\left(\alpha_{i}\right)\right) \leq \varepsilon$ for any $i=1,2, \ldots, n$ and $\mu \in M(G)$.

Proof. For $x \in G^{(0)}$, let $r(x)=\min \left\{k \in \mathbb{N} \mid \alpha^{k}(x)=x\right\}$. Since every $G$-orbit is infinite, there exists a clopen neighborhood $U_{x}$ of $x$ such that the following hold.

- $r(y)=r(x)$ for every $y \in U_{x}$,

- $\mu\left(U_{x}\right) \leq \varepsilon / r(x)$ for any $\mu \in M(G)$.

- $U_{x}, \alpha\left(U_{x}\right), \ldots, \alpha^{r(x)-1}\left(U_{x}\right)$ are mutually disjoint.

Set $V_{x}=U_{x} \cup \alpha\left(U_{x}\right) \cup \cdots \cup \alpha^{r(x)-1}\left(U_{x}\right)$. Then $V_{x}$ is $\alpha$-invariant and $\mu\left(V_{x}\right)=r(x) \mu\left(U_{x}\right) \leq \varepsilon$. We can find finitely many such clopen sets $V_{x}$, say $V_{1}, V_{2}, \ldots, V_{n}$, so that they cover $G^{(0)}$. Define $W_{i}$ inductively by $W_{1}=V_{1}$ and $W_{i}=V_{i} \backslash\left(W_{1} \cup W_{2} \cup \cdots \cup W_{i-1}\right)$. Put

$$
\alpha_{i}(x)= \begin{cases}\alpha(x) & x \in W_{i} \\ x & \text { otherwise }\end{cases}
$$

It is easy to verify that $\alpha_{1}, \alpha_{2}, \ldots, \alpha_{n}$ satisfy the requirement.

Lemma 4.4. Suppose that $G$ is almost finite and every $G$-orbit is infinite. For any $\alpha \in[[G]]$ and $\varepsilon>0$, there exist $\alpha_{1}, \alpha_{2}, \ldots, \alpha_{n} \in[[G]]$ such that $\alpha=\alpha_{1} \alpha_{2} \ldots \alpha_{n}$ and $\mu\left(\operatorname{supp}\left(\alpha_{i}\right)\right) \leq \varepsilon$ for any $i=1,2, \ldots, n$ and $\mu \in M(G)$.

Proof. This follows immediately from the lemmas above.

Lemma 4.5. Suppose that $G$ is almost finite and minimal. Let $N$ be a non-trivial subgroup of $[[G]]$ normalized by $D([[G]])$. Then there exists $\tau \in N \backslash\{1\}$ such that $\operatorname{supp}(\tau) \neq G^{(0)}$.

Proof. Take $\sigma \in N \backslash\{1\}$. There exists a non-empty clopen set $A \subset G^{(0)}$ such that $A \cap \sigma(A)=\emptyset$ and $A \cup \sigma(A) \neq G^{(0)}$. Since $G$ is minimal, there exists $\alpha \in D([[G]]) \backslash\{1\}$ such that $\operatorname{supp}(\alpha) \subset A$. Then $\tau=\alpha \sigma \alpha^{-1} \sigma^{-1}$ is in $N \backslash\{1\}$ and $\operatorname{supp}(\tau) \subset A \cup \sigma(A) \neq G^{(0)}$.

Lemma 4.6. Suppose that $G$ is almost finite and minimal. Let $N$ be a non-trivial subgroup of $[[G]]$ normalized by $D([[G]])$. If $\tau \in N$ satisfies $\operatorname{supp}(\tau) \neq G^{(0)}$, then $\alpha \tau \alpha^{-1} \in N$ for all $\alpha \in[[G]]$.

Proof. By [31, Lemma 6.8], there exists $c>0$ such that $\mu(\operatorname{supp}(\tau))<1-c$ for all $\mu \in M(G)$. Let $\alpha \in[[G]]$. By virtue of Lemma 4.4, it suffices to show $\alpha \tau \alpha^{-1} \in N$ when $\mu(\operatorname{supp}(\alpha)) \leq c$ for any $\mu \in M(G)$. It follows from [31, Lemma 6.7] that there exists $\sigma \in[[G]]$ such that $\sigma(\operatorname{supp}(\alpha)) \cap \operatorname{supp}(\tau)=\emptyset$. Therefore $\sigma \alpha^{-1} \sigma^{-1}$ commutes with $\tau$. Since $[\alpha, \sigma]=\alpha \sigma \alpha^{-1} \sigma^{-1}$ is in $D([[G]])$ and $N$ is normalized by $D([[G]])$, we get

$$
\alpha \tau \alpha^{-1}=\alpha\left(\sigma \alpha^{-1} \sigma^{-1}\right) \tau\left(\sigma \alpha \sigma^{-1}\right) \alpha^{-1} \in N
$$

which completes the proof.

The proof of the following theorem is inspired by that of [1, Theorem 3.4]. 
Theorem 4.7. Suppose that $G$ is almost finite and minimal. Then any non-trivial subgroup of $[[G]]$ normalized by the commutator subgroup $D([[G]])$ contains $D([[G]])$. In particular, $D([[G]])$ is simple.

Proof. Let $N \subset[[G]]$ be a non-trivial subgroup normalized by $D([[G]])$. By Lemma 4.5, there exists $\tau \in N \backslash\{1\}$ such that $\operatorname{supp}(\tau) \neq G^{(0)}$. Let $A \subset G^{(0)}$ be a non-empty clopen set such that $A \cap \tau(A)=\emptyset$. By [31, Lemma 6.8], there exists $c>0$ such that $\mu(A)>c$ for any $\mu \in M(G)$. We call $\alpha \in[[G]]$ small when $\mu(\operatorname{supp}(\alpha))<c / 2$ for any $\mu \in M(G)$. Notice that if $\alpha \in[[G]]$ is small, then

$$
\mu(\operatorname{supp}([\alpha, \sigma]))=\mu\left(\operatorname{supp}\left(\alpha \sigma \alpha^{-1} \sigma^{-1}\right)\right) \leq \mu(\operatorname{supp}(\alpha) \cup \sigma(\operatorname{supp}(\alpha)))<c<1
$$

holds for any $\sigma \in[[G]]$ and $\mu \in M(G)$, and hence $\operatorname{supp}([\alpha, \sigma]) \neq G^{(0)}$.

First, we claim that the commutator $[\alpha, \beta]$ belongs to $N$ for any small $\alpha, \beta \in[[G]]$. By [31, Lemma 6.7], we can find $\sigma \in[[G]]$ such that $\sigma(\operatorname{supp}(\alpha) \cup \operatorname{supp}(\beta)) \subset A$. From Lemma 4.6. we get $\tilde{\tau}=\sigma^{-1} \tau \sigma \in N$. Since $\tilde{\tau}(\operatorname{supp}(\alpha))$ and $\operatorname{supp}(\beta)$ are disjoint, $\tilde{\tau} \alpha \tilde{\tau}^{-1}$ commutes with $\beta$. Hence

$$
[\alpha, \beta]=\alpha \beta \alpha^{-1} \beta^{-1}=\alpha\left(\tilde{\tau} \alpha^{-1} \tilde{\tau}^{-1}\right) \beta\left(\tilde{\tau} \alpha \tilde{\tau}^{-1}\right) \alpha^{-1} \beta^{-1}=[[\alpha, \tilde{\tau}], \beta] .
$$

As $\operatorname{supp}(\tilde{\tau})=\sigma^{-1}(\operatorname{supp}(\tau)) \neq G^{(0)}$, by using Lemma 4.6 again, we also have $\alpha \tilde{\tau} \alpha^{-1} \in N$, and hence $[\alpha, \tilde{\tau}] \in N$. As mentioned above, $\operatorname{supp}([\alpha, \tilde{\tau}]) \neq G^{(0)}$ because $\alpha$ is small. Therefore, by Lemma 4.6, we can conclude $[\alpha, \beta] \in N$.

Next, we claim that the commutator $[\alpha, \beta]$ belongs to $N$ if $\beta$ is small. By Lemma 4.4 there exist $\alpha_{1}, \alpha_{2}, \ldots, \alpha_{n} \in[[G]]$ such that $\alpha=\alpha_{1} \alpha_{2} \ldots \alpha_{n}$ and $\alpha_{i}$ is small for all $i=1,2, \ldots, n$. Suppose that $\left[\alpha_{1} \alpha_{2} \ldots \alpha_{k-1}, \beta\right] \in N$ is known. By the claim above, $\left[\alpha_{k}, \beta\right] \in N$. Besides, we can apply Lemma 4.6 for $\left[\alpha_{k}, \beta\right]$, because $\operatorname{supp}\left(\left[\alpha_{k}, \beta\right]\right) \neq G^{(0)}$. Then

$$
\left[\alpha_{1} \alpha_{2} \ldots \alpha_{k}, \beta\right]=\alpha_{1} \alpha_{2} \ldots \alpha_{k-1}\left[\alpha_{k}, \beta\right]\left(\alpha_{1} \alpha_{2} \ldots \alpha_{k-1}\right)^{-1}\left[\alpha_{1} \alpha_{2} \ldots \alpha_{k-1}, \beta\right]
$$

is also in $N$. By induction, we obtain $[\alpha, \beta] \in N$.

Finally, we would like to show that $[\alpha, \beta]$ belongs to $N$ for any $\alpha, \beta \in[[G]]$. By Lemma 4.4, there exist $\beta_{1}, \beta_{2}, \ldots, \beta_{m} \in[[G]]$ such that $\beta=\beta_{1} \beta_{2} \ldots \beta_{m}$ and $\beta_{i}$ is small for all $i=1,2, \ldots, m$. Suppose that $\left[\alpha, \beta_{1} \beta_{2} \ldots \beta_{k-1}\right] \in N$ is known. By the claim above, $\left[\alpha, \beta_{k}\right] \in N$. Besides, we can apply Lemma 4.6 for $\left[\alpha, \beta_{k}\right]$, because $\operatorname{supp}\left(\left[\alpha, \beta_{k}\right]\right) \neq G^{(0)}$. Then

$$
\left[\alpha, \beta_{1} \beta_{2} \ldots \beta_{k}\right]=\left[\alpha, \beta_{1} \beta_{2} \ldots \beta_{k-1}\right] \beta_{1} \beta_{2} \ldots \beta_{k-1}\left[\alpha, \beta_{k}\right]\left(\beta_{1} \beta_{2} \ldots \beta_{k-1}\right)^{-1}
$$

is also in $N$. By induction, we obtain $[\alpha, \beta] \in N$. Hence the commutator subgroup $D([[G]])$ is contained in $N$.

Remark 4.8. In general, we do not know what the abelianization $[[G]] / D([[G]])$ is. In [31, Theorem 7.5], it was shown that the index map $I:[[G]] \rightarrow H_{1}(G)$ is surjective when $G$ is almost finite. Thus $[[G]] / D([[G]])$ at least has $H_{1}(G) \cong[[G]] /[[G]]_{0}$ as its quotient. For a minimal action $\varphi: \mathbb{Z} \curvearrowright X$ of $\mathbb{Z}$ on a Cantor set $X$, it is known that $\left.\left[\left[G_{\varphi}\right]\right]_{0} / D\left(\left[\left[G_{\varphi}\right]\right]\right]\right)$ is isomorphic to $H_{0}\left(G_{\varphi}\right) \otimes \mathbb{Z}_{2}$ (see [30, Theorem 4.8] and the remark following it). 


\subsection{Purely infinite groupoids}

Throughout this subsection, we let $G$ be an essentially principal étale groupoid whose unit space is a Cantor set. In this subsection, we would like to show that the commutator subgroup $D([[G]])$ of the topological full group $[[G]]$ is simple when $G$ is purely infinite and minimal (Theorem 4.16). We first introduce the notion of purely infinite groupoids. This definition is inspired by the work of M. Rørdam and A. Sierakowski [39].

Definition 4.9. (1) A clopen set $A \subset G^{(0)}$ is said to be properly infinite if there exist compact open $G$-sets $U, V \subset G$ such that $s(U)=s(V)=A, r(U) \cup r(V) \subset A$ and $r(U) \cap r(V)=\emptyset$.

(2) We say that $G$ is purely infinite if every clopen set $A \subset G^{(0)}$ is properly infinite.

It is easy to see that if $G$ is purely infinite, then for any clopen set $Y \subset G^{(0)}$ the reduction $G \mid Y$ is also purely infinite. It is also clear that if $G^{(0)}$ is properly infinite, then there does not exist a $G$-invariant probability measure. When $G$ is purely infinite, in essentially the same way as [39, Theorem 4.1], one can prove that the reduced groupoid $C^{*}$-algebra $C_{r}^{*}(G)$ is purely infinite.

The following is a generalization of [28, Theorem 2.5].

Proposition 4.10. Suppose that $G^{(0)}$ is properly infinite. The group $[[G]]$ contains a subgroup isomorphic to the free product $\mathbb{Z}_{2} * \mathbb{Z}_{3}$. In particular, $[[G]]$ is not amenable.

Proof. Since $G^{(0)}$ is properly infinite, there exist compact open $G$-sets $U, V \subset G$ such that $s(U)=s(V)=G^{(0)}$ and $r(U) \cap r(V)=\emptyset$. Let $A=r(U)$ and $B=r(V)$. Define $\alpha, \beta \in[[G]]$ by

$$
\alpha(x)=\left\{\begin{array}{ll}
\pi_{V}\left(\pi_{U}^{-1}(x)\right) & x \in A \\
\pi_{U}\left(\pi_{V}^{-1}(x)\right) & x \in B \\
x & \text { otherwise, }
\end{array} \quad \beta(x)= \begin{cases}\pi_{V}\left(\pi_{U}^{-1}\left(\pi_{U}^{-1}(x)\right)\right) & x \in r(U U) \\
\pi_{U}(x) & x \in B \\
\pi_{U}\left(\pi_{U}\left(\pi_{V}^{-1}\left(\left(\pi_{U}^{-1}(x)\right)\right)\right)\right) & x \in r(U V) \\
x & \text { otherwise }\end{cases}\right.
$$

Evidently $\alpha^{2}=1, \beta^{3}=1, \alpha(A)=B, \beta(B) \subset A$ and $\beta^{2}(B) \subset A$. The table-tennis lemma (see [19, II.B.24], for instance) implies that the subgroup generated by $\alpha$ and $\beta$ is isomorphic to the free product $\mathbb{Z}_{2} * \mathbb{Z}_{3}$.

Proposition 4.11. The following conditions are mutually equivalent.

(1) $G$ is purely infinite and minimal.

(2) For any clopen sets $A, B \subset G^{(0)}$ with $B \neq \emptyset$, there exists a compact open $G$-set $U \subset G$ such that $s(U)=A$ and $r(U) \subset B$.

(3) For any clopen sets $A, B \subset G^{(0)}$ with $A \neq G^{(0)}$ and $B \neq \emptyset$, there exists $\alpha \in[[G]]$ such that $\alpha(A) \subset B$.

Proof. (1) $\Rightarrow(2)$ There exist compact open $G$-sets $U, V \subset G$ such that $s(U)=s(V)=B$, $r(U) \cup r(V) \subset B$ and $r(U) \cap r(V)=\emptyset$, because $B$ is properly infinite. Define $V_{1}, V_{2}, \ldots$ inductively by $V_{1}=U, V_{n+1}=V V_{n}$. Then $V_{n}$ are compact open $G$-sets which satisfy 
$s\left(V_{n}\right)=B, r\left(V_{n}\right) \subset B$ and $r\left(V_{n}\right) \cap r\left(V_{m}\right)=\emptyset$ for $n \neq m$. Since $G$ is minimal, we can find compact open $G$-sets $W_{1}, W_{2}, \ldots, W_{n}$ such that $r\left(W_{i}\right) \subset B, A=s\left(W_{1}\right) \cup s\left(W_{2}\right) \cup \cdots \cup s\left(W_{n}\right)$ and $s\left(W_{i}\right) \cap s\left(W_{j}\right)=\emptyset$ for $i \neq j$. Define a compact open $G$-set $W \subset G$ by

$$
W=\bigcup_{i=1}^{n} V_{i} W_{i} .
$$

Clearly we have $s(W)=A$ and $r(W) \subset B$.

$(2) \Rightarrow(3)$ First, we assume that $B \backslash A$ is not empty. By (2), there exists a compact open $G$-set $U \subset G$ such that $s(U)=A$ and $r(U) \subset B \backslash A$. Then

$$
V=U \cup U^{-1} \cup\left(G^{(0)} \backslash(s(U) \cup r(U))\right)
$$

is a compact open $G$-set satisfying $s(V)=r(V)=G^{(0)}$. Hence $\pi_{V}$ belongs to $[[G]]$. It is easy to see $\pi_{V}(A) \subset B$. Next, we assume that $B \backslash A$ is empty, thus $B \subset A$. By the argument above, we can find $\alpha_{1}, \alpha_{2} \in[[G]]$ such that $\alpha_{1}(A) \subset G^{(0)} \backslash A$ and $\alpha_{2}\left(G^{(0)} \backslash A\right) \subset B$. Then $\alpha=\alpha_{2} \alpha_{1}$ meets the requirement.

$(3) \Rightarrow(1)$ Clearly (3) implies minimality of $G$. Let $A \subset G^{(0)}$ be a non-empty clopen subset. Choose mutually disjoint non-empty clopen sets $B_{0}, B_{1}, B_{2} \subset A$. By (3), there exist $\alpha_{1}, \alpha_{2} \in[[G]]$ satisfying $\alpha_{1}\left(A \backslash B_{0}\right) \subset B_{1}$ and $\alpha_{2}\left(B_{0} \cup B_{1}\right) \subset B_{2}$. Let $U_{1}, U_{2}$ be compact open $G$-sets such that $\alpha_{1}=\pi_{U_{1}}$ and $\alpha_{2}=\pi_{U_{2}}$. Define compact open $G$-sets $V_{1}$ and $V_{2}$ by

$$
V_{1}=B_{0} \cup U_{1}\left(A \backslash B_{0}\right) \text { and } \quad V_{2}=U_{2} V_{1} .
$$

It is not so hard to see $s\left(V_{1}\right)=s\left(V_{2}\right)=A, r\left(V_{1}\right) \subset B_{0} \cup B_{1}$ and $r\left(V_{2}\right) \subset B_{2}$. The proof is complete.

Remark 4.12. Let $X$ be a Cantor set and let $\varphi: \Gamma \curvearrowright X$ be an action of a countable discrete group $\Gamma$ by homeomorphisms. The action $\varphi$ is said to be $n$-filling in the sense of P. Jolissaint and G. Robertson [22] if for any non-empty clopen sets $A_{1}, A_{2}, \ldots, A_{n}$ of $X$, there exist $g_{1}, g_{2}, \ldots, g_{n} \in \Gamma$ such that $\varphi_{g_{1}}\left(A_{1}\right) \cup \cdots \cup \varphi_{g_{n}}\left(A_{n}\right)=X$. We can see that if $\varphi$ is $n$-filling, then the transformation groupoid $G_{\varphi}=\Gamma \times X$ is purely infinite and minimal. Indeed, one can verify condition (2) of the proposition above as follows. We identify the unit space $G^{(0)}$ with $X$. Let $A, B \subset X$ be non-empty clopen sets. We would like to construct a compact open $G_{\varphi}$-set $U \subset G_{\varphi}$ such that $s(U)=A$ and $r(U) \subset B$. Let $B_{1}, B_{2}, \ldots, B_{n} \subset B$ be mutually disjoint non-empty clopen sets. Since $\varphi$ is $n$-filling, there exist $g_{1}, g_{2}, \ldots, g_{n} \in \Gamma$ such that $\varphi_{g_{1}}\left(B_{1}\right) \cup \cdots \cup \varphi_{g_{n}}\left(B_{n}\right)=X$. Define $C_{1}, C_{2}, \ldots, C_{n}$ inductively by

$$
C_{1}=B_{1} \cap \varphi_{g_{1}}^{-1}(A) \quad \text { and } \quad C_{k+1}=B_{k+1} \cap \varphi_{g_{k+1}}^{-1}\left(A \backslash\left(\varphi_{g_{1}}\left(C_{1}\right) \cup \cdots \cup \varphi_{g_{k}}\left(C_{k}\right)\right)\right) .
$$

Then $C_{k}$ are mutually disjoint and $A$ equals the disjoint union $\varphi_{g_{1}}\left(C_{1}\right) \cup \cdots \cup \varphi_{g_{n}}\left(C_{n}\right)$. It follows that $U=\bigcup\left\{g_{k}^{-1}\right\} \times \varphi_{g_{k}}\left(C_{k}\right)$ is a compact open $G$-set with $s(U)=A$ and $r(U) \subset B$.

Lemma 4.13. Suppose that $G$ is purely infinite and minimal.

(1) For any clopen set $A, B \subset G^{(0)}$ with $A \neq G^{(0)}$ and $B \neq \emptyset$, there exists $\alpha \in[[G]]$ such that $\alpha(A) \subset B$ and $A \cup \operatorname{supp}(\alpha) \neq G^{(0)}$. 
(2) For any clopen set $A, B \subset G^{(0)}$ with $A \neq G^{(0)}$ and $B \neq \emptyset$, there exists $\alpha \in D([[G]])$ such that $\alpha(A) \subset B$.

Proof. (1) Take a clopen set $C$ so that $C \neq G^{(0)}, A \subset C, C \backslash A \neq \emptyset$ and $B \cap C \neq \emptyset$. The reduction $G \mid C=r^{-1}(C) \cap s^{-1}(C)$ is purely infinite and minimal. By Proposition 4.11 (3), there exists $\alpha \in[[G \mid C]]$ such that $\alpha(A) \subset B \cap C$. Letting $\alpha(x)=x$ for $x \notin C$, we may regard $\alpha$ as an element of $[[G]]$ and obtain $A \cup \operatorname{supp}(\alpha) \subset C \neq G^{(0)}$.

(2) By (1), we can find $\alpha \in[[G]]$ such that $\alpha(A) \subset B$ and $A \cup \operatorname{supp}(\alpha) \neq G^{(0)}$. By Proposition 4.11 (3), there exists $\sigma \in[[G]]$ such that

$$
\sigma(A \cup \operatorname{supp}(\alpha)) \subset G^{(0)} \backslash(A \cup \operatorname{supp}(\alpha)) .
$$

Then we have $\left(\alpha \sigma \alpha^{-1} \sigma^{-1}\right)(A)=\alpha(A) \subset B$.

Lemma 4.14. Suppose that $G$ is purely infinite and minimal. Let $N \subset[[G]]$ be a nontrivial subgroup normalized by $D([[G]])$. For any $\tau \in N$, there exist $\tau_{1}, \tau_{2} \in N$ such that $\operatorname{supp}\left(\tau_{1}\right) \neq G^{(0)}, \operatorname{supp}\left(\tau_{2}\right) \neq G^{(0)}$ and $\tau=\tau_{1} \tau_{2}$.

Proof. It suffices to show the lemma when $\operatorname{supp}(\tau)=G^{(0)}$. We can find a non-empty clopen set $A \subset G^{(0)}$ such that $A \cap \tau(A)=\emptyset$ and $A \cup \tau(A) \neq G^{(0)}$. Choose a non-empty clopen set $B$ so that $B \cap A=\emptyset, B \cap \tau(A)=\emptyset$ and $A \cup \tau(A) \cup B \neq G^{(0)}$. There exists $\sigma_{0} \in[[G]]$ such that $\sigma_{0}(\tau(A)) \subset B$, because $G$ is purely infinite and minimal. Define $\sigma_{1}, \sigma_{2} \in[[G]]$ by

$$
\sigma_{1}(x)=\left\{\begin{array}{ll}
\tau(x) & x \in A \\
\tau^{-1}(x) & x \in \tau(A) \\
x & \text { otherwise }
\end{array} \quad \text { and } \quad \sigma_{2}(x)= \begin{cases}\sigma_{0}(x) & x \in \tau(A) \\
\sigma_{0}^{-1}(x) & x \in \sigma_{0}(\tau(A)) \\
x & \text { otherwise }\end{cases}\right.
$$

Then $\sigma=\left[\sigma_{2}, \sigma_{1}\right] \in D([[G]])$ satisfies $\operatorname{supp}(\sigma) \subset A \cup \tau(A) \cup B$ and $\sigma(x)=\tau(x)$ for $x \in A$.

By Lemma $4.13(2)$, there exists $\alpha \in D([[G]])$ such that $\alpha(A \cup \tau(A) \cup B) \subset A$. Since $N$ is normalized by $D([[G]])$ and $\tau$ is in $N$, we have

$$
\tau_{1}=\alpha^{-1}\left[\alpha \sigma \alpha^{-1}, \tau\right] \alpha \in N .
$$

In addition, as

$$
\operatorname{supp}\left(\tau \alpha \sigma \alpha^{-1} \tau^{-1}\right)=\tau(\alpha(\operatorname{supp}(\sigma))) \subset \tau(\alpha(A \cup \tau(A) \cup B)) \subset \tau(A)
$$

and $A \cap \tau(A)=\emptyset$, for any $x \in A$ we get

$$
\tau_{1}(x)=\left(\sigma \alpha^{-1} \tau \alpha \sigma^{-1} \alpha^{-1} \tau^{-1} \alpha\right)(x)=\left(\sigma \alpha^{-1} \alpha\right)(x)=\sigma(x)=\tau(x) .
$$

Also, one has $\operatorname{supp}\left(\tau_{1}\right) \subset \alpha^{-1}(A \cup \tau(A)) \neq G^{(0)}$. Let $\tau_{2}=\tau_{1}^{-1} \tau$. Then $\tau=\tau_{1} \tau_{2}$ is a desired decomposition.

Lemma 4.15. Suppose that $G$ is purely infinite and minimal. Let $N \subset[[G]]$ be a nontrivial subgroup normalized by $D([[G]])$. For any $\tau \in N$ and $\alpha \in[[G]]$, we have $\alpha \tau \alpha^{-1} \in N$. 
Proof. First, we assume $\operatorname{supp}(\alpha) \neq G^{(0)}$ and $\operatorname{supp}(\tau) \neq G^{(0)}$. By Proposition 4.11 (3), there exists $\sigma \in[[G]]$ such that $\sigma(\operatorname{supp}(\alpha)) \cap \operatorname{supp}(\tau)=\emptyset$. Then

$$
\alpha \tau \alpha^{-1}=[\alpha, \sigma] \tau[\alpha, \sigma]^{-1} \in N,
$$

because $\sigma \alpha \sigma^{-1}$ commutes with $\tau$.

Let us consider the general case. Let $\tau \in N$ and $\alpha \in[[G]]$. By Lemma 4.14, there exist $\tau_{1}, \tau_{2} \in N$ such that $\operatorname{supp}\left(\tau_{1}\right) \neq G^{(0)}, \operatorname{supp}\left(\tau_{2}\right) \neq G^{(0)}$ and $\tau=\tau_{1} \tau_{2}$. Similarly, there exist $\alpha_{1}, \alpha_{2} \in[[G]]$ such that $\operatorname{supp}\left(\alpha_{1}\right) \neq G^{(0)}, \operatorname{supp}\left(\alpha_{2}\right) \neq G^{(0)}$ and $\alpha=\alpha_{1} \alpha_{2}$. (It is easy to find such a decomposition for an element in $[[G]]$.) It follows from the proof above that

$$
\alpha \tau \alpha^{-1}=\left(\alpha_{1} \alpha_{2} \tau_{1} \alpha_{2}^{-1} \alpha_{1}^{-1}\right)\left(\alpha_{1} \alpha_{2} \tau_{2} \alpha_{2}^{-1} \alpha_{1}^{-1}\right) \in N .
$$

We are now ready to prove the main theorem of this subsection.

Theorem 4.16. Suppose that $G$ is purely infinite and minimal. Then any non-trivial subgroup of $[[G]]$ normalized by the commutator subgroup $D([[G]])$ contains $D([[G]])$. In particular, $D([[G]])$ is simple.

Proof. Let $N$ be a non-trivial subgroup of $[[G]]$ normalized by $D([[G]])$. Let $\tau \in N \backslash\{1\}$. There exists a non-empty clopen set $A \subset G^{(0)}$ such that $A \cap \tau(A)=\emptyset$.

We would like to show that $[\alpha, \beta]$ is in $N$ for any $\alpha, \beta \in[[G]]$. First, we assume $\operatorname{supp}(\alpha) \neq G^{(0)}$ and $\operatorname{supp}(\beta) \neq G^{(0)}$. By Lemma $4.13(1)$, we can find $\gamma \in[[G]]$ such that $\gamma(\operatorname{supp}(\alpha)) \cap \operatorname{supp}(\beta)=\emptyset$ and $\operatorname{supp}(\alpha) \cup \operatorname{supp}(\gamma) \neq G^{(0)}$. There exists $\sigma \in[[G]]$ such that $\sigma(\operatorname{supp}(\alpha) \cup \operatorname{supp}(\gamma)) \subset A$. By Lemma 4.15, $\tilde{\tau}=\sigma^{-1} \tau \sigma$ is in $N$. It is easy to see

$$
(\operatorname{supp}(\alpha) \cup \operatorname{supp}(\gamma)) \cap \tilde{\tau}(\operatorname{supp}(\alpha) \cup \operatorname{supp}(\gamma))=\emptyset,
$$

and so $\left(\tilde{\tau} \gamma \tilde{\tau}^{-1}\right)(x)=x$ for any $x \in \operatorname{supp}(\alpha)$. Hence $\tilde{\gamma}=[\gamma, \tilde{\tau}]$ satisfies $\tilde{\gamma}(\operatorname{supp}(\alpha)) \cap$ $\operatorname{supp}(\beta)=\emptyset$. It follows that $\tilde{\gamma} \alpha \tilde{\gamma}^{-1}$ commutes with $\beta$. Moreover, by Lemma 4.15, $\tilde{\gamma}$ is in $N$, too. Therefore

$$
[\alpha, \beta]=\alpha \beta \alpha^{-1} \beta^{-1}=\alpha\left(\tilde{\gamma} \alpha \tilde{\gamma}^{-1}\right) \beta\left(\tilde{\gamma} \alpha^{-1} \tilde{\gamma}^{-1}\right) \alpha^{-1} \beta^{-1}=[[\alpha, \tilde{\gamma}], \beta]
$$

is in $N$.

Next, we would like to show $[\alpha, \beta] \in N$ when $\operatorname{supp}(\beta) \neq G^{(0)}$. We can find $\alpha_{1}, \alpha_{2} \in[[G]]$ such that $\operatorname{supp}\left(\alpha_{1}\right) \neq G^{(0)}, \operatorname{supp}\left(\alpha_{2}\right) \neq G^{(0)}$ and $\alpha=\alpha_{1} \alpha_{2}$. By the proof above, $\left[\alpha_{1}, \beta\right]$ and $\left[\alpha_{2}, \beta\right]$ are in $N$. It follows from Lemma 4.15 that

$$
[\alpha, \beta]=\left[\alpha_{1} \alpha_{2}, \beta\right]=\alpha_{1}\left[\alpha_{2}, \beta\right] \alpha_{1}^{-1}\left[\alpha_{1}, \beta\right]
$$

belongs to $N$.

Finally, let us show $[\alpha, \beta] \in N$. We can find $\beta_{1}, \beta_{2} \in[[G]]$ such that $\operatorname{supp}\left(\beta_{1}\right) \neq G^{(0)}$, $\operatorname{supp}\left(\beta_{2}\right) \neq G^{(0)}$ and $\beta=\beta_{1} \beta_{2}$. By the proof above, $\left[\alpha, \beta_{1}\right]$ and $\left[\alpha, \beta_{2}\right]$ are in $N$. It follows from Lemma 4.15 that

$$
[\alpha, \beta]=\left[\alpha, \beta_{1} \beta_{2}\right]=\left[\alpha, \beta_{1}\right] \beta_{1}\left[\alpha, \beta_{2}\right] \beta_{1}^{-1}
$$

belongs to $N$, as desired. 
Remark 4.17. In view of Theorem 4.16, it is a natural problem to determine the abelianization $[[G]] / D([[G]])$. In Theorem [5.2, it will be shown that the index map $I:[[G]] \rightarrow$ $H_{1}(G)$ is surjective when $G$ is purely infinite. Thus $[[G]] / D([[G]])$ at least has $H_{1}(G) \cong$ $[[G]] /[[G]]_{0}$ as its quotient. In general, however, we do not know what the quotient group $[[G]]_{0} / D([[G]])$ is. When $G$ arises from a one-sided irreducible shift of finite type, we will prove that $[[G]] / D([[G]])$ is isomorphic to $\left(H_{0}(G) \otimes \mathbb{Z}_{2}\right) \oplus H_{1}(G)$ (Corollary 6.24 (1)).

\section{Surjectivity of the index map}

Throughout this section, we let $G$ be an essentially principal étale groupoid whose unit space is a Cantor set. In this section we will show that the index map $I:[[G]] \rightarrow H_{1}(G)$ is surjective when $G$ is purely infinite (Theorem [5.2).

Lemma 5.1. Suppose that $G$ is purely infinite. For any clopen sets $A_{1}, A_{2}, \ldots, A_{n} \subset G^{(0)}$, there exist compact open $G$-sets $U_{1}, U_{2}, \ldots, U_{n}$ such that $s\left(U_{i}\right)=A_{i}$ for all $i$ and the sets $r\left(U_{i}\right)$ are mutually disjoint.

Proof. The proof is by induction on $n$. Suppose that the lemma is known for $n-1$. Let $A_{1}, A_{2}, \ldots, A_{n} \subset G^{(0)}$ be clopen sets. There exist compact open $G$-sets $U_{1}, U_{2}, \ldots, U_{n-1}$ such that $s\left(U_{i}\right)=A_{i}$ for all $i$ and the sets $r\left(U_{i}\right)$ are mutually disjoint. Set $B_{i}=r\left(U_{i}\right) \cap A_{n}$ for $i=1,2, \ldots, n-1$. By pure infiniteness of $G$, we can find compact open $G$-sets $V_{i}$ and $W_{i}$ such that $s\left(V_{i}\right)=s\left(W_{i}\right)=B_{i}, r\left(V_{i}\right) \cup r\left(W_{i}\right) \subset B_{i}$ and $r\left(V_{i}\right) \cap r\left(W_{i}\right)=\emptyset$. Define compact open $G$-sets $U_{1}^{\prime}, U_{2}^{\prime}, \ldots, U_{n-1}^{\prime}$ by

$$
U_{i}^{\prime}=\left(\left(r\left(U_{i}\right) \backslash B_{i}\right) \cup V_{i} B_{i}\right) U_{i} .
$$

Let

$$
U_{n}=W_{1} B_{1} \cup W_{2} B_{2} \cup \cdots \cup W_{n-1} B_{n-1} \cup\left(A_{n} \backslash\left(B_{1} \cup B_{2} \cup \cdots \cup B_{n-1}\right)\right) .
$$

Then $U_{1}^{\prime}, U_{2}^{\prime}, \ldots, U_{n-1}^{\prime}, U_{n}$ meet the requirement.

Theorem 5.2. Suppose that $G$ is purely infinite. The index map $I:[[G]] \rightarrow H_{1}(G)$ is surjective.

Proof. Let $\delta_{1}: C_{c}(G, \mathbb{Z}) \rightarrow C\left(G^{(0)}, \mathbb{Z}\right)$ and $\delta_{2}: C_{c}\left(G^{(2)}, \mathbb{Z}\right) \rightarrow C_{c}(G, \mathbb{Z})$ be as in 31 , Section 3]. The homology group $H_{1}(G)$ is $\operatorname{Ker} \delta_{1} / \operatorname{Im} \delta_{2}$. For $f \in \operatorname{Ker} \delta_{1}$, we denote its equivalence class in $H_{1}(G)$ by $[f]$. For a subset $C \subset G$, we let $1_{C}$ denote the characteristic function of $C$.

Let $f \in \operatorname{Ker} \delta_{1}$. We would like to show that there exists a compact open $G$-set $V$ such that $s(V)=r(V)=G^{(0)}$ and $[f]=\left[1_{V}\right]$. By [31, Lemma $\left.7.3(4)\right]$, we may assume that there exist compact open $G$-sets $C_{1}, C_{2}, \ldots, C_{n}$ such that $f=1_{C_{1}}+1_{C_{2}}+\cdots+1_{C_{n}}$. Since $f$ is in $\operatorname{Ker} \delta_{1}$, one has

$$
\sum_{i=1}^{n} 1_{r\left(C_{i}\right)}=\sum_{j=1}^{n} 1_{s\left(C_{j}\right)}
$$

Hence there exist clopen sets $A_{i j} \subset G^{(0)}$ for $i, j=1,2, \ldots, n$ satisfying

$$
\sum_{i=1}^{n} 1_{A_{i j}}=1_{r\left(C_{j}\right)} \text { and } \sum_{j=1}^{n} 1_{A_{i j}}=1_{s\left(C_{i}\right)} .
$$


By the lemma above, we can find compact open $G$-sets $U_{1}, U_{2}, \ldots, U_{n}$ such that $s\left(U_{i}\right)=$ $r\left(C_{i}\right)$ for all $i$ and the sets $r\left(U_{i}\right)$ are mutually disjoint. Put

$$
V_{i j}=U_{i} C_{i} A_{i j} U_{j}^{-1} \text {. }
$$

Then $V_{i j}$ is a compact open $G$-set which satisfies

$$
r\left(V_{i j}\right)=U_{i} C_{i} A_{i j} U_{j}^{-1} U_{j} A_{i j} C_{i} U_{i}^{-1}=U_{i} C_{i} A_{i j} r\left(C_{j}\right) A_{i j} C_{i} U_{i}^{-1}=r\left(U_{i} C_{i} A_{i j}\right)
$$

and

$$
s\left(V_{i j}\right)=U_{j} A_{i j} C_{i}^{-1} U_{i}^{-1} U_{i} C_{i} A_{i j} U_{j}^{-1}=U_{j} A_{i j} C_{i}^{-1} r\left(C_{i}\right) C_{i} A_{i j} U_{j}^{-1}=s\left(A_{i j} U_{j}^{-1}\right) .
$$

Therefore $V=\bigcup_{i, j} V_{i j}$ is a compact open $G$-set satisfying

$$
r(V)=\bigcup_{i=1}^{n} r\left(U_{i} C_{i}\right)=\bigcup_{i=1}^{n} r\left(U_{i}\right)=\bigcup_{j=1}^{n} s\left(U_{j}^{-1}\right)=s(V) .
$$

By using [31, Lemma 7.3 (1)] repeatedly, we obtain

$$
\begin{aligned}
{\left[_{V}\right] } & =\left[\sum_{i, j} 1_{V_{i j}}\right]=\left[\sum_{i, j} 1_{U_{i} C_{i} A_{i j} U_{j}^{-1}}\right] \\
& =\left[\sum_{i, j}\left(1_{U_{i} C_{i} A_{i j}}+1_{A_{i j} U_{j}^{-1}}\right)\right] \\
& =\left[\sum_{i} 1_{U_{i} C_{i}}+\sum_{j} 1_{U_{j}^{-1}}\right] \\
& =\left[\sum_{i}\left(1_{U_{i}}+1_{C_{i}}\right)+\sum_{j} 1_{U_{j}^{-1}}\right]
\end{aligned}
$$

in $H_{1}(G)$. This, together with [31, Lemma $\left.7.3(4)\right]$, implies $\left[1_{V}\right]=[f]$ in $H_{1}(G)$. Hence $\tilde{V}=V \cup\left(G^{(0)} \backslash s(V)\right)$ is a desired $G$-set.

We will use the following lemma in Section 6.5. For $f \in C\left(G^{(0)}, \mathbb{Z}\right)$, we denote its equivalence class in $H_{0}(G)$ by $[f]_{G}$.

Lemma 5.3. Suppose that $G$ is purely infinite. For any $h \in H_{0}(G)$, there exists a nonempty clopen set $A$ such that $\left[1_{A}\right]_{G}=h$.

Proof. There exists $f \in C\left(G^{(0)}, \mathbb{Z}\right)$ such that $[f]_{G}=h$. We can find non-empty clopen sets $C_{1}, C_{2}, \ldots, C_{n}$ and $D_{1}, D_{2}, \ldots, D_{m}$ such that

$$
f=\sum_{i=1}^{n} 1_{C_{i}}-\sum_{j=1}^{m} 1_{D_{j}}
$$


From Lemma [5.1, there exist compact open $G$-sets $U_{1}, U_{2}, \ldots, U_{n}$ and $V_{1}, V_{2}, \ldots, V_{m}$ such that $s\left(U_{i}\right)=C_{i}, s\left(V_{j}\right)=D_{j}$ for all $i, j$ and the sets $r\left(U_{i}\right), r\left(V_{j}\right)$ are mutually disjoint. Let

$$
C=\bigcup_{i=1}^{n} r\left(U_{i}\right) \quad \text { and } \quad D=\bigcup_{j=1}^{m} r\left(V_{j}\right) .
$$

Then $[f]_{G}=\left[1_{C}\right]_{G}-\left[1_{D}\right]_{G}$. Since $G$ is purely infinite, there exist compact open $G$-sets $U, V \subset G$ such that $s(U)=s(V)=D, r(U) \cup r(V) \subset D$ and $r(U) \cap r(V)=\emptyset$. Put $E=D \backslash(r(U) \cup r(V))$. One has

$$
\left[1_{E}\right]_{G}=\left[1_{D}\right]_{G}-\left[1_{r(U)}\right]_{G}-\left[1_{r(V)}\right]_{G}=\left[1_{D}\right]_{G}-\left[1_{D}\right]_{G}-\left[1_{D}\right]_{G}=-\left[1_{D}\right]_{G},
$$

which implies

$$
h=[f]_{G}=\left[1_{C}\right]_{G}-\left[1_{D}\right]_{G}=\left[1_{C}\right]_{G}+\left[1_{E}\right]_{G}=\left[1_{C \cup E}\right]_{G},
$$

as desired.

\section{Groupoids from shifts of finite type}

In this section we study topological full groups of étale groupoids arising from shifts of finite type. We refer the reader to [10, 25] for background material on symbolic dynamical systems.

\subsection{Preliminaries}

Let $(\mathcal{V}, \mathcal{E})$ be a finite directed graph, where $\mathcal{V}$ is a finite set of vertices and $\mathcal{E}$ is a finite set of edges. For $e \in \mathcal{E}, i(e)$ denotes the initial vertex of $e$ and $t(e)$ denotes the terminal vertex of $e$. Let $M=(M(\xi, \eta))_{\xi, \eta \in \mathcal{V}}$ be the adjacency matrix of $(\mathcal{V}, \mathcal{E})$, that is,

$$
M(\xi, \eta)=\#\{e \in \mathcal{E} \mid i(e)=\xi, t(e)=\eta\} .
$$

We assume that $M$ is irreducible (i.e. for all $\xi, \eta \in \mathcal{V}$ there exists $n \in \mathbb{N}$ such that $\left.M^{n}(\xi, \eta)>0\right)$ and that $M$ is not a permutation matrix. Define

$$
X=\left\{\left(x_{k}\right)_{k \in \mathbb{N}} \in \mathcal{E}^{\mathbb{N}} \mid t\left(x_{k}\right)=i\left(x_{k+1}\right) \quad \forall k \in \mathbb{N}\right\} .
$$

With the product topology, $X$ is a Cantor set (see condition (I) defined in [10]). Define a surjective continuous map $\sigma: X \rightarrow X$ by

$$
\sigma(x)_{k}=x_{k+1} \quad k \in \mathbb{N}, x=\left(x_{k}\right)_{k} \in X .
$$

In other words, $\sigma$ is the (one-sided) shift on $X$. It is easy to see that $\sigma$ is a local homeomorphism. The dynamical system $(X, \sigma)$ is called the one-sided irreducible shift of finite type associated with the graph $(\mathcal{V}, \mathcal{E})$.

The étale groupoid $G$ for $(X, \sigma)$ is given by

$$
G=\left\{(x, n, y) \in X \times \mathbb{Z} \times X \mid \exists k, l \in \mathbb{N}, n=k-l, \sigma^{k}(x)=\sigma^{l}(y)\right\} .
$$


The topology of $G$ is generated by the sets $\left\{(x, k-l, y) \in G \mid x \in A, y \in B, \sigma^{k}(x)=\right.$ $\left.\sigma^{l}(y)\right\}$, where $A, B \subset X$ are open and $k, l \in \mathbb{N}$. Two elements $(x, n, y)$ and $\left(x^{\prime}, n^{\prime}, y^{\prime}\right)$ in $G$ are composable if and only if $y=x^{\prime}$, and the multiplication and the inverse are

$$
(x, n, y) \cdot\left(y, n^{\prime}, y^{\prime}\right)=\left(x, n+n^{\prime}, y^{\prime}\right), \quad(x, n, y)^{-1}=(y,-n, x) .
$$

We identify $X$ with the unit space $G^{(0)}$ via $x \mapsto(x, 0, x)$.

A multiindex $\mu=\left(e_{1}, e_{2}, \ldots, e_{k}\right)$ with $e_{j} \in \mathcal{E}$ is called a word. We denote by $|\mu|$ the length $k$ of $\mu$ and write $i(\mu)=i\left(e_{1}\right), t(\mu)=t\left(e_{k}\right)$. Every $e \in \mathcal{E}$ is regarded as a word of length one. When $\mu=\left(e_{1}, e_{2}, \ldots, e_{k}\right)$ and $\nu=\left(f_{1}, f_{2}, \ldots, f_{l}\right)$ are words, we write $\mu \nu$ for the word $\mu \nu=\left(e_{1}, \ldots, e_{k}, f_{1}, \ldots, f_{l}\right)$ of length $k+l$. A word $\mu=\left(e_{1}, e_{2}, \ldots, e_{k}\right)$ is said to be admissible if $t\left(e_{j}\right)=i\left(e_{j+1}\right)$ for every $j=1,2, \ldots, k-1$. For a word $\mu=\left(e_{1}, e_{2}, \ldots, e_{k}\right)$,

$$
C_{\mu}=\left\{\left(x_{n}\right)_{n \in \mathbb{N}} \in X \mid x_{j}=e_{j} \quad \forall j=1,2, \ldots, k\right\}
$$

is a clopen subset of $X$ and is called a cylinder set. Clearly, $C_{\mu}$ is non-empty if and only if $\mu$ is admissible. For any two cylinder sets $C_{\mu}$ and $C_{\nu}$, we have $C_{\mu} \cap C_{\nu}=\emptyset, C_{\mu} \subset C_{\nu}$ or $C_{\mu} \supset C_{\nu}$. For $\xi \in \mathcal{V}$, we set

$$
D_{\xi}=\left\{\left(x_{n}\right)_{n \in \mathbb{N}} \in X \mid i\left(x_{1}\right)=\xi\right\} .
$$

For words $\mu$ and $\nu$ with $t(\mu)=t(\nu)$, we define a compact open $G$-set $U_{\mu, \nu}$ by

$$
U_{\mu, \nu}=\left\{(x,|\mu|-|\nu|, y) \in G \mid \sigma^{|\mu|}(x)=\sigma^{|\nu|}(y), x \in C_{\mu}, y \in C_{\nu}\right\} .
$$

The subsets $U_{\mu, \nu}$ form a base for the topology of $G$.

Lemma 6.1. For any non-empty clopen set $Y \subset X$, the étale groupoid $G \mid Y$ is purely infinite and minimal.

Proof. By the remark following Definition 4.9, we may assume $Y=X$. We check condition (2) of Proposition 4.11, Let $A$ and $B$ be non-empty clopen subsets of $X$. Write $A$ and $B$ as disjoint unions $A=\bigcup_{\mu \in I} C_{\mu}$ and $B=\bigcup_{\nu \in J} C_{\nu}$ of non-empty cylinder sets, respectively. By dividing up the cylinder sets $C_{\nu}$ if necessary, we may assume that $\# J$ is not less than $\# I$. Let $f: I \rightarrow J$ be an injection. Since the graph $(\mathcal{V}, \mathcal{E})$ is irreducible, for each $\mu \in I$, we can find an admissible word $\mu^{\prime}$ such that $C_{\mu^{\prime}} \subset C_{f(\mu)}$ and $t\left(\mu^{\prime}\right)=t(\mu)$. Set

$$
U=\bigcup_{\mu \in I} U_{\mu^{\prime}, \mu}
$$

Then $U$ is a compact open $G$-set satisfying $s(U)=A$ and $r(U) \subset B$.

Let us recall the homology groups $H_{n}(G)$ from [31, Section 4]. The étale groupoid $G$ has an open subgroupoid

$$
K=\{(x, 0, y) \in G\} .
$$

It is well-known that $K$ is an AF (approximately finite) groupoid (see [37, Defintion III.1.1] or [31, Definition 2.2] for the definition of AF groupoids) and that $H_{0}(K)$ is isomorphic to the inductive limit

$$
\underset{\lim }{\longrightarrow}\left(\mathbb{Z}^{\mathcal{V}} \stackrel{M^{t}}{\longrightarrow} \mathbb{Z}^{\mathcal{V}} \stackrel{M^{t}}{\longrightarrow} \ldots\right)
$$


where $M^{t}$ is the transpose of $M$ and is thought of as a homomorphism from $\mathbb{Z}^{\mathcal{V}}$ to $\mathbb{Z}^{\mathcal{V}}$. This group is called the dimension group of the subshift $(X, \sigma)$. Notice that $K$ is not necessary minimal. Indeed, $K$ is minimal if and only if the subshift $(X, \sigma)$ is topologically mixing. For $f \in C(X, \mathbb{Z})$, we denote its equivalence class in $H_{0}(K)$ by $[f]_{K}$. There exists an automorphism $\delta: H_{0}(K) \rightarrow H_{0}(K)$ such that

$$
\left[1_{r(U)}\right]_{K}=\delta^{-n}\left(\left[1_{s(U)}\right]_{K}\right)
$$

holds for any $n \in \mathbb{Z}$ and any compact open $G$-set $U$ contained in $\{(x, n, y) \in G\}$. Then, by virtue of [31, Theorem 4.14], we have

$$
\begin{gathered}
H_{0}(G) \cong \operatorname{Coker}(\mathrm{id}-\delta) \cong \operatorname{Coker}\left(\mathrm{id}-M^{t}\right), \\
H_{1}(G) \cong \operatorname{Ker}(\mathrm{id}-\delta) \cong \operatorname{Ker}\left(\mathrm{id}-M^{t}\right)
\end{gathered}
$$

and $H_{n}(G)=0$ for $n \geq 2$. In particular, $H_{0}(G)$ is a finitely generated abelian group and $H_{1}(G)$ is the torsion-free part of $H_{0}(G)$. We remark that Coker(id $-M^{t}$ ) is called the Bowen-Franks group of $M$ ([25, Definition 7.4.15]). For $f \in C(X, \mathbb{Z})$, we denote its equivalence class in $H_{0}(G)$ by $[f]_{G}$.

K. Matsumoto [26, 27] obtained the following classification theorem (see also [31, Theorem 5.1]). Actually, only the case of $Y_{i}=X_{i}$ was considered in [26, 27], but the proof works in the general case.

Theorem 6.2 ([26, Theorem 1.1], [27, Theorem 1.1]). For $i=1,2$, let $\left(\mathcal{V}_{i}, \mathcal{E}_{i}\right)$ be a finite directed graph and suppose that the adjacency matrix $M_{i}$ is irreducible and is not a permutation matrix. Let $\left(X_{i}, \sigma_{i}\right)$ be the one-sided shift associated with $\left(\mathcal{V}_{i}, \mathcal{E}_{i}\right)$ and let $G_{i}$ be the étale groupoid for $\left(X_{i}, \sigma_{i}\right)$. Let $Y_{i} \subset X_{i}$ be a non-empty clopen subset. If there exists an isomorphism $\varphi: H_{0}\left(G_{1}\right) \rightarrow H_{0}\left(G_{2}\right)$ such that $\varphi\left(\left[1_{Y_{1}}\right]_{G_{1}}\right)=\left[1_{Y_{2}}\right]_{G_{2}}$ and $\operatorname{det}\left(\mathrm{id}-M_{1}^{t}\right)=\operatorname{det}\left(\mathrm{id}-M_{2}^{t}\right)$, then $G_{1} \mid Y_{1}$ is isomorphic to $G_{2} \mid Y_{2}$ as an étale groupoid.

In the theorem above, we do not know whether or not the hypothesis $\operatorname{det}\left(\mathrm{id}-M_{1}^{t}\right)=$ $\operatorname{det}\left(\mathrm{id}-M_{2}^{t}\right)$ is really necessary. If the torsion-free part of $H_{0}\left(G_{i}\right)$ is non-zero (or equivalently $\left.H_{1}\left(G_{i}\right) \neq 0\right)$, then $\operatorname{det}\left(\mathrm{id}-M_{i}^{t}\right)=0$. Thus the hypothesis $\operatorname{det}\left(\mathrm{id}-M_{1}^{t}\right)=\operatorname{det}\left(\mathrm{id}-M_{2}^{t}\right)$ automatically holds. When $H_{0}\left(G_{i}\right)$ is a torsion group (or equivalently $H_{1}\left(G_{i}\right)=0$ ), we always have $\left|\operatorname{det}\left(\mathrm{id}-M_{1}^{t}\right)\right|=\left|\operatorname{det}\left(\mathrm{id}-M_{2}^{t}\right)\right|$, and so the only issue is the sign of $\operatorname{det}\left(\mathrm{id}-M_{i}^{t}\right)$.

We also remark that the pair Coker $\left(\mathrm{id}-M^{t}\right)$ and $\operatorname{det}\left(\mathrm{id}-M^{t}\right)$ is a complete invariant of flow equivalence for two-sided irreducible shifts of finite type ([13]).

Remark 6.3. We would like to note a close connection between the topological full group $[[G]]$ and Thompson's groups. I wish to thank R. Grigorchuk, who drew my attention to this connection. For background on Thompson's groups, see the survey article [7] and the references therein. In 1965 Richard Thompson gave the first example of a finitely presented infinite simple group. G. Higman [20] and K. S. Brown [4] later introduced infinite families $F_{n, r} \subset T_{n, r} \subset V_{n, r}$ of finitely presented infinite simple groups generalizing Thompson's example. Notice that $V_{n, r}$ is written $G_{n, r}$ in [4]. Here we describe $F_{n, r}$ and $V_{n, r}$ as subgroups of PL (piecewise linear) maps on an interval.

Let $n \in \mathbb{N} \backslash\{1\}$ and $r \in \mathbb{N}$. Let $F_{n, r}$ be the set of all PL homeomorphisms $f:[0, r] \rightarrow$ $[0, r]$ with finitely many singularities such that all singularities of $f$ are in $\mathbb{Z}[1 / n]$ and the derivative of $f$ at any non-singular point is $n^{k}$ for some $k \in \mathbb{Z}$. It is easy to see that 
$F_{n, r}$ is closed under composition and forms a group. It is known that $F_{n, r}$ is independent of $r$ up to isomorphism, the commutator subgroup $D\left(F_{n, r}\right)$ is simple, the abelianization $F_{n, r} / D\left(F_{n, r}\right)$ is $\mathbb{Z}^{n}$, and $F_{n, r}$ is finitely presented ([4]). It is an outstanding open problem whether the group $F_{n, r}$ is amenable. The group $V_{n, r}$ is defined to be the group of all right continuous PL bijections $f:[0, r) \rightarrow[0, r)$ with finitely many singularities such that all singularities of $f$ are in $\mathbb{Z}[1 / n]$, the derivative of $f$ at any non-singular point is $n^{k}$ for some $k \in \mathbb{Z}$ and $f$ maps $\mathbb{Z}[1 / n] \cap[0, r)$ to itself. We call $V_{n, r}$ the Higman-Thompson groups. It is known that $V_{n, r}$ is finitely presented, the commutator subgroup $D\left(V_{n, r}\right)$ is simple, and the abelianization $V_{n, r} / D\left(V_{n, r}\right)$ is trivial when $n$ is even and is $\mathbb{Z}_{2}$ when $n$ is odd ([4]). Also, $V_{n, r}$ contains non-abelian free groups. For $n_{1}, n_{2}, r_{1}, r_{2}$, the groups $V_{n_{1}, r_{1}}$ and $V_{n_{2}, r_{2}}$ are isomorphic if and only if $n_{1}=n_{2}$ and $\operatorname{gcd}\left(n_{1}-1, r_{1}\right)=\operatorname{gcd}\left(n_{2}-1, r_{2}\right)$ ([36]). It is also possible to describe $F_{n, r} \subset V_{n, r}$ as groups of homeomorphisms of the Cantor set.

Let $(X, \sigma)$ be the full shift over $n$ symbols and let $G$ be the étale groupoid associated to $(X, \sigma)$. The $C^{*}$-algebra $C_{r}^{*}(G)$ is the Cuntz algebra $\mathcal{O}_{n} . \mathrm{V}$. V. Nekrashevych [34] proved that the Higman-Thompson group $V_{n, 1}$ is identified with a certain subgroup of the unitary group $U\left(\mathcal{O}_{n}\right)$ ([34, Proposition 9.6]). This identification yields an isomorphism between the Higman-Thompson group $V_{n, 1}$ and the topological full group $[[G]]$. Therefore, when $(X, \sigma)$ is a shift of finite type which is not necessarily a full shift, $[[G]]$ can be thought of as a generalization of $V_{n, 1}$. We will prove that $[[G]]$ is of type $\mathrm{F}_{\infty}$ in later subsections. In Section 6.7.1, we revisit the étale groupoids of full shifts.

\subsection{Equivalence between clopen subsets}

We use the notation of the preceding subsection. As mentioned above, $H_{0}(G)$ is isomorphic to Coker $\left(\mathrm{id}-M^{t}\right)$. This isomorphism is described as follows. For $\xi \in \mathcal{V}$, we consider the clopen set $D_{\xi}=\left\{x \in X \mid i\left(x_{1}\right)=\xi\right\}$. The map sending $\xi \mapsto\left[1_{D_{\xi}}\right]_{G}$ gives rise to a homomorphism from $\mathbb{Z}^{\mathcal{V}}$ to $H_{0}(G)$. It is well-known that this homomorphism is surjective and its kernel is $\left(\mathrm{id}-M^{t}\right) \mathbb{Z}^{\mathcal{V}}$ (see [9, Proposition 3.1], [31, Theorem 4.14]). Thus, it induces $H_{0}(G) \cong \operatorname{Coker}\left(\mathrm{id}-M^{t}\right)$.

Theorem 6.4. For non-empty clopen sets $A, B \subset X$, the following conditions are equivalent.

(1) $\left[1_{A}\right]_{G}=\left[1_{B}\right]_{G}$ in $H_{0}(G)$.

(2) There exists a compact open $G$-set $U$ such that $s(U)=A$ and $r(U)=B$.

Proof. The implication from (2) to (1) is immediate from the definition of $H_{0}(G)$. We show that (1) implies (2). Write $A$ and $B$ as disjoint unions $A=\bigcup_{\mu \in I} C_{\mu}$ and $B=\bigcup_{\nu \in J} C_{\nu}$ of non-empty cylinder sets $C_{\mu}, C_{\nu}$, respectively. By dividing up a cylinder set if necessary, we may assume that there exist $\mu_{0} \in I$ and $\nu_{0} \in J$ such that $t\left(\mu_{0}\right)=\zeta=t\left(\nu_{0}\right)$. We define $a, b \in \mathbb{Z}^{\mathcal{V}}$ by

$$
a(\xi)=\#\{\mu \in I \mid t(\mu)=\xi\}, \quad b(\xi)=\#\{\nu \in J \mid t(\nu)=\xi\} \quad \forall \xi \in \mathcal{V} .
$$

Since $\left[1_{A}\right]_{G}$ equals $\left[1_{B}\right]_{G}, a-b \in \mathbb{Z}^{\mathcal{V}}$ is in the image of id $-M^{t}$. Therefore, there exist $c, d \in \mathbb{N}^{\mathcal{V}}$ such that $a+\left(M^{t}-\mathrm{id}\right) c=b+\left(M^{t}-\mathrm{id}\right) d$. By dividing the clopen set $D_{\zeta}$ 
into cylinder sets, we can find a finite set $L$ of admissible words such that $D_{\zeta}$ equals the disjoint union $\bigcup_{\lambda \in L} C_{\lambda}$ and

$$
\#\{\lambda \in L \mid t(\lambda)=\xi\} \geq \max \{c(\xi), d(\xi)\} \quad \forall \xi \in \mathcal{V} .
$$

Define $I^{\prime}$ and $J^{\prime}$ by

$$
I^{\prime}=\left(I \backslash\left\{\mu_{0}\right\}\right) \cup\left\{\mu_{0} \lambda \mid \lambda \in L\right\} \quad \text { and } \quad J^{\prime}=\left(J \backslash\left\{\nu_{0}\right\}\right) \cup\left\{\nu_{0} \lambda \mid \lambda \in L\right\} .
$$

Then we have $A=\bigcup_{\mu \in I^{\prime}} C_{\mu}$ and $B=\bigcup_{\nu \in J^{\prime}} C_{\nu}$. Also,

$$
a^{\prime}(\xi)=\#\left\{\mu \in I^{\prime} \mid t(\mu)=\xi\right\} \quad \text { and } \quad b^{\prime}(\xi)=\#\left\{\nu \in J^{\prime} \mid t(\nu)=\xi\right\}
$$

still satisfy $a^{\prime}+\left(M^{t}-\mathrm{id}\right) c=b^{\prime}+\left(M^{t}-\mathrm{id}\right) d$, because $a^{\prime}-a=b^{\prime}-b$. For each $\xi \in \mathcal{V}$, we can find a finite subset $I_{\xi}^{\prime} \subset I^{\prime}$ such that $t(\mu)=\xi$ for all $\mu \in I_{\xi}^{\prime}$ and $\# I_{\xi}^{\prime}=c(\xi)$. Define $I^{\prime \prime}$ by

$$
I^{\prime \prime}=\left(I^{\prime} \backslash \bigcup_{\xi \in \mathcal{V}} I_{\xi}^{\prime}\right) \cup \bigcup_{\xi \in \mathcal{V}}\left\{\mu e \mid \mu \in I_{\xi}^{\prime}, e \in \mathcal{E}, i(e)=\xi\right\} .
$$

Then we have $A=\bigcup_{\mu \in I^{\prime \prime}} C_{\mu}$ and

$$
a^{\prime \prime}(\xi)=\#\left\{\mu \in I^{\prime \prime} \mid t(\mu)=\xi\right\}=a^{\prime}(\xi)-c(\xi)+\sum_{\eta} M(\eta, \xi) c(\eta)
$$

that is, $a^{\prime \prime}=a^{\prime}+\left(M^{t}-\mathrm{id}\right) c$. Similarly, we can construct a set $J^{\prime \prime}$ of admissible words such that $B=\bigcup_{\nu \in J^{\prime \prime}} C_{\nu}$ and $b^{\prime \prime}=b^{\prime}+\left(M^{t}-\mathrm{id}\right) d$, where

$$
b^{\prime \prime}(\xi)=\#\left\{\nu \in J^{\prime \prime} \mid t(\nu)=\xi\right\} .
$$

It follows from $a^{\prime}+\left(M^{t}-\mathrm{id}\right) c=b^{\prime}+\left(M^{t}-\mathrm{id}\right) d$ that there exists a bijection $f: I^{\prime \prime} \rightarrow J^{\prime \prime}$ such that $t(\mu)=t(f(\mu))$ for every $\mu \in I^{\prime \prime}$. Then

$$
U=\bigcup_{\mu \in I^{\prime \prime}} U_{f(\mu), \mu}
$$

is a compact open $G$-set satisfying $r(U)=B$ and $s(U)=A$.

\subsection{The Haagerup property}

As in Section 6.1, we let $G$ be the étale groupoid arising from a one-sided irreducible shift of finite type $(X, \sigma)$. We use the notation of Section 6.1 . We have already seen that $[[G]]$ is not amenable (see Proposition 4.10 and Proposition 6.1). In this subsection, we would like to see that $[[G]]$ has the Haagerup property, i.e. it is a-T-menable in the sense of $\mathrm{M}$. Gromov. Indeed, this is a corollary of B. Hughes's theorem [21, Theorem 1.1], which states that any locally finitely determined group of local similarities on a compact ultrametric space has the Haagerup property. One can regard $X$ as a compact ultrametric space and introduce a suitable finite similarity structure for (cylinder sets of) $X$. Then [21, Theorem 1.1] applies to show that $[[G]]$ has the Haagerup property. But, instead of proceeding in this way, we would like to provide a self-contained succinct proof of it for the convenience of the reader. I wish to thank R. Grigorchuk, who drew my attention to this problem and to the reference [21].

We recall the notion of zipper actions from [21]. 
Definition 6.5 ([21, Defintion 5.1]). An action $\varphi: \Gamma \curvearrowright \Omega$ of a discrete group $\Gamma$ on a set $\Omega$ is called a zipper action if there exists a subset $Z \subset \Omega$ such that the following hold.

(1) For every $\alpha \in \Gamma$, the symmetric difference $\varphi_{\alpha}(Z) \triangle Z$ is finite.

(2) For every $r>0,\left\{\alpha \in \Gamma \mid \#\left(\varphi_{\alpha}(Z) \triangle Z\right) \leq r\right\}$ is finite.

Theorem 6.6 ([21, Theorem 5.3]). If a discrete group $\Gamma$ admits a zipper action, then $\Gamma$ has the Haagerup property.

In order to apply the theorem above to $\Gamma=[[G]]$, we have to construct a zipper action of $[[G]]$. Let $\Omega$ be the set of equivalence classes of non-empty compact open $G$-sets $V$ such that $s(V)$ is a cylinder set. Two such $V_{1}$ and $V_{2}$ are equivalent provided $V_{2}=V_{1} U_{\mu, \nu}$, where $s\left(V_{1}\right)=C_{\mu}$ and $s\left(V_{2}\right)=C_{\nu}$. We denote the equivalence class of $V$ in $\Omega$ by $[V]$. Define an action $\varphi:[[G]] \curvearrowright \Omega$ by $\varphi_{\alpha}([V])=[W V]$, where $W$ is the compact open $G$-set such that $\alpha=\pi_{W}$.

Theorem 6.7. The action $\varphi:[[G]] \curvearrowright \Omega$ is a zipper action. In particular, $[[G]]$ has the Haagerup property.

Proof. Define a subset $Z \subset \Omega$ by

$$
Z=\left\{\left[C_{\mu}\right] \in \Omega \mid C_{\mu} \text { is a non-empty cylinder set }\right\} .
$$

Let $\alpha=\pi_{W} \in[[G]]$. There exist admissible words $\mu_{1}, \mu_{2}, \ldots, \mu_{k}, \nu_{1}, \nu_{2}, \ldots, \nu_{k}$ such that $t\left(\mu_{i}\right)=t\left(\nu_{i}\right)$ for all $i=1,2, \ldots, k$ and $W$ equals the disjoint union $\bigcup_{i=1}^{k} U_{\mu_{i}, \nu_{i}}$. Suppose that there exists $L \subset\{1,2, \ldots, k\}$ such that $\bigcup_{i \in L} U_{\mu_{i}, \nu_{i}}$ equals $U_{\mu, \nu}$ for some admissible words $\mu, \nu$ with $t(\mu)=t(\nu)$. Then we may replace $\bigcup_{i \in L} U_{\mu_{i}, \nu_{i}}$ with $U_{\mu, \nu}$. By repeating this procedure if necessary, we may assume that no such $L$ exists. Let $m(\alpha)=\max \left\{\left|\mu_{i}\right|,\left|\nu_{i}\right| \mid i=1,2, \ldots, k\right\}$.

We would like to estimate $\#\left(\varphi_{\alpha}(Z) \triangle Z\right)$. Let $\left[C_{\lambda}\right] \in Z$. Then $\varphi_{\alpha}\left(\left[C_{\lambda}\right]\right)=\left[W C_{\lambda}\right]$ is in $Z$ if and only if there exists $i$ such that $C_{\lambda}$ is contained in $C_{\nu_{i}}$. Hence, if $\left[C_{\lambda}\right]$ is in $Z \backslash \varphi_{\alpha}^{-1}(Z)$, then $C_{\lambda}$ is not contained in any $C_{\nu_{i}}$, and so in particular

$$
|\lambda|<\max \left\{\left|\nu_{i}\right| \mid i=1,2, \ldots, k\right\} \leq m(\alpha) .
$$

Therefore $\#\left(Z \backslash \varphi_{\alpha}^{-1}(Z)\right)=\#\left(\varphi_{\alpha}(Z) \backslash Z\right)$ does not exceed the number of admissible words of length less than $m(\alpha)$, and hence is finite. In the same way, we can show $\#\left(Z \backslash \varphi_{\alpha}(Z)\right)$ is finite, which implies condition (1) of Definition 6.5. Next, let us estimate $\#\left(\varphi_{\alpha}(Z) \triangle Z\right)$ from below. Without loss of generality, we may assume $m(\alpha)=\left|\nu_{1}\right|$. Let $\nu_{1}=\left(e_{1}, e_{2}, \ldots, e_{m(\alpha)}\right)$. Put $\lambda_{j}=\left(e_{1}, e_{2}, \ldots, e_{j}\right)$ for $j=1,2, \ldots, m(\alpha)-1$. Then $C_{\lambda_{j}}$ is not contained in any $C_{\nu_{i}}$. It follows that $\varphi_{\alpha}\left(\left[C_{\lambda_{j}}\right]\right)$ is not in $Z$, and so we get

$$
\#\left(\varphi_{\alpha}(Z) \triangle Z\right) \geq \#\left(\varphi_{\alpha}(Z) \backslash Z\right) \geq m(\alpha)-1 \text {. }
$$

Hence, in order to prove condition (2) of Definition 6.5, for given $r>0$, it suffices to show that the set of $\alpha \in[[G]]$ such that $m(\alpha)<r$ is finite. Clearly there exist finitely many partitions of $X$ into cylinder sets $C_{\mu}$ satisfying $|\mu|<r$. Hence the number of $\alpha=\pi_{W} \in[[G]]$ for which there exist $\mu_{1}, \mu_{2}, \ldots, \mu_{k}$ and $\nu_{1}, \nu_{2}, \ldots, \nu_{k}$ such that $\left|\mu_{i}\right|<r$, $\left|\nu_{i}\right|<r$ and $W=\bigcup_{i=1}^{k} U_{\mu_{i}, \nu_{i}}$ is at most finite. The proof is complete.

For any clopen set $Y \subset X,[[G \mid Y]]$ is a subgroup of $[[G]]$. It follows from the theorem above that $[[G \mid Y]]$ also has the Haagerup property. 


\subsection{Kernel of the index map}

As in Section 6.1, we let $G$ be the étale groupoid arising from a one-sided irreducible shift of finite type $(X, \sigma)$. We use the notation of Section 6.1. Fix a non-empty clopen subset $Y \subset X$. In this subsection, we would like to show that $[[G \mid Y]]_{0}$ is finitely generated (Theorem 6.11).

First we need to write down the index map $I:[[G \mid Y]] \rightarrow H_{1}(G \mid Y)$ explicitly. Any $\alpha \in[[G \mid Y]]$ extends to an element of $[[G]]$ by letting $\alpha(x)=x$ for $x \in X \backslash Y$, and so we may regard $[[G \mid Y]]$ as a subgroup of $[[G]]$. Since $G$ is minimal, the inclusion $G \mid Y \hookrightarrow G$ induces isomorphisms between $H_{n}(G \mid Y)$ and $H_{n}(G)$ (see Proposition 3.5 and Theorem 3.6 of [31]). These observations yield the diagram

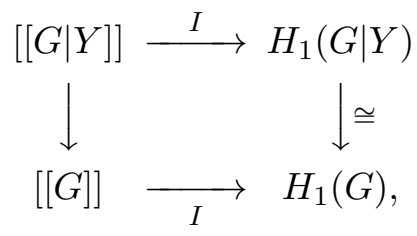

which becomes commutative by the definition of the index map. From now on, we identify $H_{1}(G \mid Y)$ with $H_{1}(G)$. As mentioned in Section 6.1, $H_{1}(G)$ is isomorphic to $\operatorname{Ker}(\mathrm{id}-\delta)$, which is a subgroup of $H_{0}(K)$. For $\alpha \in[[G \mid Y]]$, we would like to describe $I(\alpha) \in H_{1}(G \mid Y) \cong H_{1}(G)$ as an element of $\operatorname{Ker}($ id $-\delta)$. Take a compact open $G \mid Y$-set $U$ such that $\alpha=\pi_{U}$. For $n \in \mathbb{Z}$, we put

$$
S(\alpha, n)=\{x \in Y \mid(\alpha(x), n, x) \in U\} .
$$

Note that $S(\alpha, n)$ is empty except for finitely many $n$. We have

$$
\left[1_{Y}\right]_{K}=\sum_{n \in \mathbb{Z}}\left[1_{S(\alpha, n)}\right]_{K}
$$

in $H_{0}(K)$, because $\{S(\alpha, n) \mid n \in \mathbb{Z}\}$ is a clopen partition of $Y$. Since $\{\alpha(S(\alpha, n)) \mid n \in \mathbb{Z}\}$ is also a clopen partition of $Y$, one obtains

$$
\left[1_{Y}\right]_{K}=\sum_{n \in \mathbb{Z}}\left[1_{\alpha(S(\alpha, n))}\right]_{K}=\sum_{n \in \mathbb{Z}} \delta^{-n}\left(\left[1_{S(\alpha, n)}\right]_{K}\right)
$$

in $H_{0}(K)$. Therefore, we get

$$
\sum_{n \in \mathbb{Z}}\left(\mathrm{id}-\delta^{-n}\right)\left(\left[1_{S(\alpha, n)}\right]_{K}\right)=0 .
$$

For $n \in \mathbb{Z}$, we define homomorphisms $\delta^{(n)}: H_{0}(K) \rightarrow H_{0}(K)$ by

$$
\delta^{(n)}= \begin{cases}\mathrm{id}+\delta+\cdots+\delta^{n-1} & n>0 \\ 0 & n=0 \\ -\left(\delta^{-1}+\delta^{-2}+\cdots+\delta^{n}\right) & n<0\end{cases}
$$

so that $(\mathrm{id}-\delta) \delta^{(n)}=\mathrm{id}-\delta^{n}$ hold. It follows from (6.1) that

$$
\sum_{n \in \mathbb{Z}}(\mathrm{id}-\delta)\left(\delta^{(-n)}\left(\left[1_{S(\alpha, n)}\right]_{K}\right)\right)=0,
$$


which means that $\sum_{n \in \mathbb{Z}} \delta^{(-n)}\left(\left[1_{S(\alpha, n)}\right]_{K}\right)$ is in $\operatorname{Ker}(\mathrm{id}-\delta)$. The proof of [31, Theorem 4.14] immediately implies the following.

Lemma 6.8. In the setting above, $I(\alpha) \in H_{1}(G \mid Y) \cong H_{1}(G)$ is identified with

$$
\sum_{n \in \mathbb{Z}} \delta^{(-n)}\left(\left[1_{S(\alpha, n)}\right]_{K}\right) \in \operatorname{Ker}(\mathrm{id}-\delta)
$$

In what follows, we regard $[[K \mid Y]]$ as a subgroup of $[[G \mid Y]]$. As mentioned in Section 6.1, $K$ is an AF groupoid, and so is $K \mid Y$. Hence, by [30, Proposition 3.2], [[K|Y]] is a locally finite group. More precisely, $[[K \mid Y]]$ is written as an increasing union of finite direct sums of symmetric groups. In particular, $[[K \mid Y]]$ is contained in $[[G \mid Y]]_{0}=\operatorname{Ker} I$. For two clopen sets $A, B \subset X$, it is well-known that $\left[1_{A}\right]_{K}=\left[1_{B}\right]_{K}$ in $H_{0}(K)$ if and only if there exists a compact open $K$-set $U$ such that $s(U)=A$ and $r(U)=B$. For $\alpha \in[[G \mid Y]]$, it is also easy to see that $\alpha$ belongs to $[[K \mid Y]]$ if and only if $S(\alpha, 0)=Y$.

We introduce the set of transpositions. We call $\alpha \in[[G \mid Y]]$ a transposition if there exists a clopen set $A \subset Y$ such that $A \cap \alpha(A)=\emptyset, \operatorname{supp}(\alpha)=A \cup \alpha(A)$ and $\alpha^{2}=1$. By Theorem 6.4, if non-empty clopen sets $A, B \subset Y$ satisfy $A \cap B=\emptyset$ and $\left[1_{A}\right]_{G}=\left[1_{B}\right]_{G}$, then there exists a transposition $\alpha \in[[G \mid Y]]$ such that $\alpha(A)=B$ and $\operatorname{supp}(\alpha)=A \cup B$. We let $\mathcal{T}$ denote the set of all transpositions. By [31, Lemma 7.3], $\mathcal{T}$ is contained in $[[G \mid Y]]_{0}=\operatorname{Ker} I$. It is also easy to see that the group $[[K \mid Y]]$ is generated by $\mathcal{T} \cap[[K \mid Y]]$. Below we will see that $[[G \mid Y]]_{0}$ is generated by $\mathcal{T}$ (Lemma 6.10 and Theorem 6.11).

Lemma 6.9. Let $A, B \subset Y$ be clopen sets and let $n \in \mathbb{Z}$. If $\delta^{n}\left(\left[1_{A}\right]_{K}\right)=\left[1_{B}\right]_{K}$ in $H_{0}(K)$, then there exists a compact open $G$-set $U$ such that $r(U)=A, s(U)=B$ and $U \subset\{(x, n, y) \in G\}$. Furthermore, if $A$ and $B$ are disjoint, then there exists $\tau \in \mathcal{T}$ such that $S(\tau,-n)=A, S(\tau, n)=B, \tau(A)=B$ and $\operatorname{supp}(\tau)=A \cup B$.

Proof. The case $n=0$ is trivial as mentioned above. We may assume without loss of generality that $n$ is positive. Write $B$ as a disjoint union $B=\bigcup_{\mu \in I} C_{\mu}$ of non-empty cylinder sets. For each $\mu \in I$, there exists an admissible word $\mu^{\prime}$ such that $t\left(\mu^{\prime}\right)=i(\mu)$ and $\left|\mu^{\prime}\right|=n$. Then

$$
\sum_{\mu \in I}\left[1_{C_{\mu^{\prime} \mu}}\right]_{K}=\sum_{\mu \in I} \delta^{-n}\left(\left[1_{C_{\mu}}\right]_{K}\right)=\delta^{-n}\left(\left[1_{B}\right]_{K}\right)=\left[1_{A}\right]_{K}
$$

in $H_{0}(K)$. Since $K$ is an AF groupoid, we can find compact open $K$-sets $V_{\mu}$ such that $s\left(V_{\mu}\right)=C_{\mu^{\prime} \mu}$ and $A$ equals the disjoint union $\bigcup_{\mu} r\left(V_{\mu}\right)$. Then

$$
U=\bigcup_{\mu \in I} V_{\mu} U_{\mu^{\prime} \mu, \mu}
$$

is a desired compact open $G$-set.

Assume further that $A$ and $B$ are disjoint. Clearly

$$
V=U \cup U^{-1} \cup(Y \backslash(A \cup B))
$$

is a compact open $G$-set satisfying $r(V)=s(V)=Y$, and $\tau=\pi_{V}$ satisfies the requirement. 
Lemma 6.10. Suppose that $(X, \sigma)$ is topologically mixing. Then $[[G \mid Y]]_{0}$ is generated by the set $\mathcal{T}$ of transpositions.

Proof. We remark that $(X, \sigma)$ is topologically mixing if and only if the matrix $M=$ $(M(\xi, \eta))_{\xi, \eta \in \mathcal{V}}$ is primitive, that is, there exists $n \in \mathbb{N}$ such that $M^{n}(\xi, \eta)>0$ for all $\xi, \eta \in$ $\mathcal{V}([25$, Proposition 4.5.10]). Moreover, in this case the AF groupoid $K$ is minimal and has a unique $K$-invariant probability measure on $X$, say $\omega$. We can define a homomorphism $\hat{\omega}: H_{0}(K) \rightarrow \mathbb{R}$ by

$$
\hat{\omega}([f])=\int f d \omega
$$

for $f \in C(X, \mathbb{Z})$. Let $r>1$ be the Perron eigenvalue of $M$. Then it is well-known that $\hat{\omega} \circ \delta=r \hat{\omega}$ holds.

Let $\langle\mathcal{T}\rangle$ be the subgroup generated by $\mathcal{T}$. Suppose that $\alpha \in[[G \mid Y]]_{0} \backslash\{1\}$ is given. There exists a non-empty clopen set $C \subset Y$ such that $C \cap \alpha(C)=\emptyset$. We can construct $\tau \in \mathcal{T}$ such that $\tau(x)=\alpha(x)$ for all $x \in C$. Hence, by replacing $\alpha$ with $\tau \alpha$, we may assume that $\operatorname{supp}(\alpha)$ is not equal to $Y$. Let $A=\operatorname{supp}(\alpha) \varsubsetneqq Y$. Since $K$ is minimal, one has $\omega(Y)>0$ and $\omega(Y \backslash A)>0$.

By Lemma 6.8, we have

$$
\sum_{n \in \mathbb{Z}} \delta^{(-n)}\left(\left[1_{S(\alpha, n)}\right]_{K}\right)=0
$$

Set $P=\{n \in \mathbb{N} \mid S(\alpha, n) \neq \emptyset\}$ and $Q=\{n \in \mathbb{Z} \mid n<0, S(\alpha, n) \neq \emptyset\}$. Then

$$
-\sum_{n \in P} \delta^{(-n)}\left(\left[1_{S(\alpha, n)}\right]_{K}\right)=\sum_{n \in Q} \delta^{(-n)}\left(\left[1_{S(\alpha, n)}\right]_{K}\right) .
$$

Put

$$
z=\left[1_{A}\right]_{K}+\delta\left(\sum_{n \in Q} \delta^{(-n)}\left(\left[1_{S(\alpha, n)}\right]_{K}\right)\right) \in H_{0}(K) .
$$

We have

$$
\hat{\omega}(z)=\omega(A)+\sum_{n \in Q}\left(r+r^{2}+\cdots+r^{-n}\right) \omega(S(\alpha, n)) .
$$

Choose $m \in \mathbb{N}$ so that

$$
r^{-m} \omega(A)<\omega(Y \backslash A) \text { and } r^{-m} \hat{\omega}(z)<\omega(Y)
$$

hold. Then we can find a clopen set $B \subset Y \backslash A$ such that $\left[1_{B}\right]_{K}=\delta^{-m}\left(\left[1_{A}\right]_{K}\right)$. It follows from Lemma 6.9 that there exists $\tau_{0} \in \mathcal{T}$ such that $S\left(\tau_{0}, m\right)=A, S\left(\tau_{0},-m\right)=B$, $\tau_{0}(A)=B$ and $\operatorname{supp}\left(\tau_{0}\right)=A \cup B$. Set $\beta=\tau_{0} \alpha \tau_{0}$. It suffices to show that $\beta$ belongs to $\langle\mathcal{T}\rangle$. Notice that $\operatorname{supp}(\beta)=\tau_{0}(A)=B, S(\beta, n)=\tau_{0}(S(\alpha, n))$ and

$$
\left[1_{S(\beta, n)}\right]_{K}=\delta^{-m}\left(\left[1_{S(\alpha, n)}\right]_{K}\right)
$$

for every $n \in \mathbb{Z}$. Hence, by (6.2), we get

$$
-\sum_{n \in P} \delta^{(-n)}\left(\left[1_{S(\beta, n)}\right]_{K}\right)=\sum_{n \in Q} \delta^{(-n)}\left(\left[1_{S(\beta, n)}\right]_{K}\right) .
$$


We would like to construct $\gamma \in\langle\mathcal{T}\rangle$ such that $S(\gamma, n)=S(\beta, n)$ for all $n \in \mathbb{Z}$. Because

$$
\begin{aligned}
& \omega(B)+\sum_{n \in Q}\left(r+r^{2}+\cdots+r^{-n}\right) \omega(S(\beta, n)) \\
& =r^{-m}\left(\omega(A)+\sum_{n \in Q}\left(r+r^{2}+\cdots+r^{-n}\right) \omega(S(\alpha, n))\right) \\
& =r^{-m} \hat{\omega}(z)<\omega(Y),
\end{aligned}
$$

there exist mutually disjoint clopen sets $C_{n, i} \subset Y \backslash B$ for $n \in Q$ and $i=1,2, \ldots,-n$ such that

$$
\delta\left(\left[1_{S(\beta, n)}\right]_{K}\right)=\left[1_{C_{n, 1}}\right]_{K} \quad \text { and } \quad \delta\left(\left[1_{C_{n, i}}\right]_{K}\right)=\left[1_{C_{n, i+1}}\right]_{K}
$$

for each $n \in Q$ and $i=1,2, \ldots,-n-1$. By Lemma 6.9, for $n \in Q$ and $i=1,2, \ldots,-n$, we can find $\tau_{n, i} \in \mathcal{T}$ such that

$$
\begin{gathered}
S\left(\tau_{n, 1},-1\right)=S(\beta, n), \quad S\left(\tau_{n, 1}, 1\right)=C_{n, 1}, \\
\tau_{n, 1}(S(\beta, n))=C_{n, 1}, \quad \operatorname{supp}\left(\tau_{n, 1}\right)=S(\beta, n) \cup C_{n, 1}
\end{gathered}
$$

and

$$
\begin{gathered}
S\left(\tau_{n, i+1},-1\right)=C_{n, i}, \quad S\left(\tau_{n, i+1}, 1\right)=C_{n, i+1}, \\
\tau_{n, i+1}\left(C_{n, i}\right)=C_{n, i+1}, \quad \operatorname{supp}\left(\tau_{n, i+1}\right)=C_{n, i} \cup C_{n, i+1} .
\end{gathered}
$$

Define $\tau_{-} \in\langle\mathcal{T}\rangle$ by

$$
\tau_{-}=\prod_{n \in Q} \tau_{n,-n} \ldots \tau_{n, 2} \tau_{n, 1}
$$

Then we can verify

$$
S\left(\tau_{-}, n\right)=S(\beta, n) \quad \forall n \in Q, \quad S\left(\tau_{-}, 1\right)=\bigcup_{n \in Q} \bigcup_{i=1}^{-n} C_{n, i}
$$

and

$$
\tau_{-}(S(\beta, n))=C_{n,-n} \quad \forall n \in Q, \quad \operatorname{supp}\left(\tau_{-}\right)=\bigcup_{n \in Q}\left(S(\beta, n) \cup \bigcup_{i=1}^{-n} C_{n, i}\right) .
$$

Next, we would like to consider $S(\beta, n)$ for $n \in P$. By using (6.3), we have

$$
\begin{aligned}
& \sum_{n \in P}\left[1_{S(\beta, n)}\right]_{K}+\sum_{n \in P}\left(\delta^{-1}+\delta^{-2}+\cdots+\delta^{-n}\right)\left(\left[1_{S(\beta, n)}\right]_{K}\right) \\
& =\sum_{n \in P}\left[1_{S(\beta, n)}\right]_{K}-\sum_{n \in P} \delta^{(-n)}\left(\left[1_{S(\beta, n)}\right]_{K}\right) \\
& =\sum_{n \in P}\left[1_{S(\beta, n)}\right]_{K}+\sum_{n \in Q} \delta^{(-n)}\left(\left[1_{S(\beta, n)}\right]_{K}\right) \\
& =\sum_{n \in P}\left[1_{S(\beta, n)}\right]_{K}+\sum_{n \in Q}\left[1_{S(\beta, n)}\right]_{K}+\sum_{n \in Q}\left(\delta+\delta^{2}+\cdots+\delta^{-n-1}\right)\left(\left[1_{S(\beta, n)}\right]_{K}\right) \\
& =\sum_{n \in P}\left[1_{S(\beta, n)}\right]_{K}+\sum_{n \in Q}\left[1_{S(\beta, n)}\right]_{K}+\sum_{n \in Q} \sum_{i=1}^{-n-1}\left[1_{C_{n, i}}\right]_{K} .
\end{aligned}
$$


Hence we can find mutually disjoint clopen sets $D_{n, j}$ for $n \in P$ and $j=1,2, \ldots, n$ such that

$$
\delta^{-1}\left(\left[1_{S(\beta, n)}\right]_{K}\right)=\left[1_{D_{n, 1}}\right]_{K}, \quad \delta^{-1}\left(\left[1_{D_{n, j}}\right]_{K}\right)=\left[1_{D_{n, j+1}}\right]_{K}
$$

and

$$
\bigcup_{n \in P} \bigcup_{j=1}^{n} D_{n, j}=\bigcup_{n \in Q}\left(S(\beta, n) \cup \bigcup_{i=1}^{-n-1} C_{n, i}\right) .
$$

Therefore, in the same way as above, we can construct $\tau_{+} \in\langle\mathcal{T}\rangle$ such that

$$
S\left(\tau_{+}, n\right)=S(\beta, n) \quad \forall n \in P, \quad S\left(\tau_{+},-1\right)=\bigcup_{n \in Q}\left(S(\beta, n) \cup \bigcup_{i=1}^{-n-1} C_{n, i}\right)
$$

and

$$
\tau_{+}(S(\beta, n))=D_{n, n} \quad \forall n \in P, \quad \operatorname{supp}\left(\tau_{+}\right)=\bigcup_{n \in P} S(\beta, n) \cup \bigcup_{n \in Q}\left(S(\beta, n) \cup \bigcup_{i=1}^{-n-1} C_{n, i}\right) .
$$

Let $\gamma=\tau_{+} \tau_{-} \in\langle\mathcal{T}\rangle$. It is not so hard to see

$$
S(\gamma, n)=S(\beta, n) \quad \forall n \in P \cup Q, \quad \bigcup_{n \in P \cup Q} S(\gamma, n) \cup S(\gamma, 0)=Y,
$$

and so $S(\gamma, n)=S(\beta, n)$ for all $n \in \mathbb{Z}$. Then we obtain $S\left(\beta \gamma^{-1}, 0\right)=Y$, which implies $\beta \gamma^{-1} \in[[K \mid Y]]$ and hence $\beta \gamma^{-1} \in\langle\mathcal{T}\rangle$. It follows that $\beta$ belongs to $\langle\mathcal{T}\rangle$, as desired.

We are now ready to prove the main theorem of this subsection.

Theorem 6.11. The group $[[G \mid Y]]_{0}$ is generated by finitely many elements of $\mathcal{T}$.

Proof. Let $p \in \mathbb{N}$ be the period of the shift $(X, \sigma)$ or, equivalently, of the irreducible matrix $M$ ([25, Definition 4.5.4]). It follows from [25, Section 4.5] that there exists a non-empty $\sigma^{p}$-invariant clopen subset $Z \subset X$ such that $\left(Z, \sigma^{p} \mid Z\right)$ is (conjugate to) a topologically mixing shift of finite type. Clearly the groupoid $G \mid Z$ is identified with the étale groupoid arising from the shift $\left(Z, \sigma^{p} \mid Z\right)$. Since $G$ is purely infinite and minimal, there exists a compact open $G$-set $U$ satisfying $s(U)=Y$ and $r(U) \subset Z$ (see Lemma 6.1). Hence $G|s(U)=G| Y$ is isomorphic to $G \mid r(U)$ as an étale groupoid. The groupoid $G \mid r(U)$ is equal to the reduction of $G \mid Z$ to $r(U)$. Therefore, by replacing $G$ with $G \mid Z$, we may assume that $G$ arises from a topologically mixing shift of finite type.

Choose two distinct edges $p, q \in \mathcal{E}$ such that $t(p)=i(q)$. It is not so hard to see $C_{p q} \cup C_{q} \neq X$. By Proposition 4.11 (3), there exists a compact open $G$-set $U$ such that $Y=s(U)$ and $C_{p q} \cup C_{q} \subset r(U)$. It follows that $G|Y=G| s(U)$ is isomorphic to $G \mid r(U)$. By replacing $Y$ with $r(U)$, we may assume that $C_{p q} \cup C_{q}$ is contained in $Y$. Let $J$ be the set of admissible words $\mu$ such that $C_{\mu} \subset Y$. Then $p q, q \in J$.

For a word $\mu=e_{1} e_{2} \ldots e_{k}$ and $1 \leq i \leq j \leq k$, we write $\mu_{[i, j]}=e_{i} e_{i+1} \ldots e_{j}$. The clopen set $Y \subset X$ is written as a disjoint union of finitely many cylinder sets, and so there exists $l \in \mathbb{N}$ such that if $\mu \in J$ and $|\mu| \geq l$, then $\mu_{[1, l]} \in J$. Because we have assumed that the adjacency matrix $M$ is primitive, there exists $m \in \mathbb{N}$ such that every entry of the matrix $M^{m}$ is greater than 2 . We assume $m \geq l$. 
Let $\mu, \nu \in J$ be such that $t(\mu)=t(\nu)$. In Section 6.1, we introduced the compact open $G$-set $U_{\mu, \nu}$. Suppose that $C_{\mu}$ and $C_{\nu}$ are disjoint. Then

$$
V=U_{\mu, \nu} \cup U_{\mu, \nu}^{-1} \cup\left(Y \backslash\left(C_{\mu} \cup C_{\nu}\right)\right)
$$

is a compact open $G \mid Y$-set satisfying $r(V)=s(V)=Y$. We write $\gamma(\mu, \nu)=\pi_{V}$, which is an element of $\mathcal{T}$. Let $\mathcal{T}_{0}$ denote the set of all such elements $\gamma(\mu, \nu)$. Since the sets $U_{\mu, \nu}$ form a base for the topology of $G \mid Y$, any element of $\mathcal{T}$ is a product of finitely many elements of $\mathcal{T}_{0}$. Note also that $\gamma(\mu, \nu)$ equals $\gamma(\nu, \mu)$. Define the finite set $F$ by

$$
F=\left\{\gamma(\mu, \nu) \in \mathcal{T}_{0}|| \mu|=| \nu \mid \leq m+2\right\} \cup\left\{\gamma(\mu, \nu) \in \mathcal{T}_{0}|| \mu|+1=| \nu \mid \leq m+2\right\} .
$$

Let $\langle F\rangle$ be the subgroup generated by $F$. By Lemma 6.10, $[[G \mid Y]]_{0}$ is generated by $\mathcal{T}$. Hence, it suffices to prove that $\langle F\rangle$ contains $\mathcal{T}_{0}$.

First, we claim that $\langle F\rangle$ contains $\left\{\gamma(\mu, \nu) \in \mathcal{T}_{0}|| \mu|=| \nu \mid\right\}$. The proof is by induction on the length of the words. Assume that the claim is known for length $n$, where $n \geq m+2$. Let $\mu, \nu \in J$ be distinct admissible words of length $n+1$ such that $t(\mu)=t(\nu)$. We would like to show $\gamma(\mu, \nu) \in\langle F\rangle$. Since each entry of $M^{m}$ is not less than 3, we can find an admissible word $\lambda$ of length $n+1$ such that

$$
\lambda_{[1,2]}=p q, \quad t(\lambda)=t(\mu), \quad \lambda_{[3, m+2]} \neq \mu_{[3, m+2]}, \quad \lambda_{[3, m+2]} \neq \nu_{[3, m+2]} .
$$

We will show that $\gamma(\mu, \lambda)$ is in $\langle F\rangle$. By the choice of $m$, there exists an admissible word $a$ of length $n$ such that

$$
a_{[1,1]}=q, \quad a_{[m+2, n]}=\mu_{[m+3, n+1]}, \quad a_{[2, m+1]} \neq \lambda_{[3, m+2]}, \quad a_{[2, m+1]} \neq \mu_{[3, m+2]} .
$$

Set $b=\lambda_{[2, n+1]}$. Then $a, b \in J,|a|=|b|=n$ and $a \neq b$. By the induction hypothesis, $\gamma(a, b)$ belongs to $\langle F\rangle$. It follows that

$$
\gamma(q, p q) \gamma(a, b) \gamma(q, p q)=\gamma(p a, p b)=\gamma(p a, \lambda)
$$

also belongs to $\langle F\rangle$. Set $c=a_{[1, n-1]}$ and $d=\mu_{[1, n]}$. We have $p c, d \in J,|p c|=|d|=n$ and $p c \neq d$. By the induction hypothesis, $\gamma(p c, d)$ is in $\langle F\rangle$. Also $C_{\lambda} \cap C_{p c}=C_{\lambda} \cap C_{d}=\emptyset$ and $C_{p a} \subset C_{p c}$. Therefore

$$
\gamma(p c, d) \gamma(p a, \lambda) \gamma(p c, d)=\gamma(\mu, \lambda)
$$

is in $\langle F\rangle$, too. In the same way, we can show $\gamma(\nu, \lambda) \in\langle F\rangle$. Then

$$
\gamma(\mu, \lambda) \gamma(\nu, \lambda) \gamma(\mu, \lambda)=\gamma(\mu, \nu) \in\langle F\rangle
$$

which completes the induction, and the proof of the claim.

Next, we claim that $\langle F\rangle$ contains $\gamma(\mu, \nu)$ for $\mu, \nu$ with $|\mu|+1=|\nu|$. The proof is by induction on the length $|\nu|$. Assume that the claim is known for length $n$, where $n \geq m+2$. Let $\mu, \nu \in J$ be such that $t(\mu)=t(\nu),|\mu|=n,|\nu|=n+1$ and $C_{\mu} \cap C_{\nu}=\emptyset$. Since each entry of the matrix $M^{m}$ is greater than 2, we can find an admissible word $a$ of length $n$ such that

$$
a_{[1,1]}=q, \quad a_{[m+2, n]}=\nu_{[m+3, n+1]}, \quad a_{[2, m+1]} \neq \mu_{[2, m+1]}, \quad a_{[2, m+1]} \neq \nu_{[1, m]} .
$$


Let $b=a_{[1, n-1]}$ and $c=\nu_{[1, n]}$. Then $a, b, c \in J,|b|=n-1,|c|=n$ and $C_{b} \cap C_{c}=\emptyset$. By the induction hypothesis, $\gamma(b, c)$ is in $\langle F\rangle$. Note also that $C_{\mu} \cap C_{b}=C_{\mu} \cap C_{c}=\emptyset$ and $C_{a} \subset C_{b}$. We have already shown that $\gamma(\mu, a)$ is in $\langle F\rangle$. It follows that

$$
\gamma(b, c) \gamma(\mu, a) \gamma(b, c)=\gamma(\mu, \nu)
$$

is in $\langle F\rangle$, which completes the induction, and the proof of the claim.

Finally, we claim that $\langle F\rangle$ contains $\gamma(\mu, \nu)$ for $\mu, \nu$ with $|\mu|<|\nu|$. Clearly we may assume $|\mu| \geq m+1$. The proof is by induction on $n=|\nu|-|\mu|$. The case $n=1$ is

done. Assume that the claim is known for $n-1$. Let $\mu, \nu \in J$ be such that $t(\mu)=t(\nu)$, $|\mu| \geq m+1,|\nu|-|\mu|=n \geq 2$ and $C_{\mu} \cap C_{\nu}=\emptyset$. In the same way as the argument above, we can choose an admissible word $\lambda$ of length $|\mu|+1$ such that

$$
\lambda_{[1,1]}=q, \quad t(\lambda)=t(\mu), \quad \lambda_{[2, m+1]} \neq \mu_{[2, m+1]}, \quad \lambda_{[2, m+1]} \neq \nu_{[2, m+1]} .
$$

Thus, $\lambda$ is in $J$, and $C_{\mu}, C_{\lambda}$ and $C_{\nu}$ are mutually disjoint. By the induction hypothesis, $\gamma(\mu, \lambda)$ and $\gamma(\nu, \lambda)$ are in $\langle F\rangle$. Hence we get

$$
\gamma(\mu, \lambda) \gamma(\nu, \lambda) \gamma(\mu, \lambda)=\gamma(\mu, \nu) \in\langle F\rangle,
$$

thereby proving the theorem.

\subsection{Finiteness properties}

As in Section 6.1, we let $G$ be the étale groupoid arising from a one-sided irreducible shift of finite type $(X, \sigma)$. We use the notation of Section 6.1. Fix a non-empty clopen subset $Y \subset X$. In this subsection, we would like to show that $[[G \mid Y]]$ is of type $\mathrm{F}_{\infty}$. As mentioned in Section 1, this is equivalent to saying that $[[G \mid Y]]$ is of type $\mathrm{FP}_{\infty}$ and is finitely presented.

Such finiteness properties for Thompson's group $F_{2,1}$ were studied by K. S. Brown and R. Geoghegan [6]. Brown [4, 5] later extended this to the infinite families $F_{n, r} \subset T_{n, r} \subset V_{n, r}$ (see Remark 6.3). M. Stein [41] generalized these families further. Here we follow the approach of [4, 5, 41]. The following theorem, due to Brown, is the key tool. We refer the reader to [2, Section I.4] for the definition of a $\Gamma$-complex.

Theorem 6.12 ([4, Corollary 3.3]). Let $\Gamma$ be a group. Suppose that $\Gamma$ admits a contractible $\Gamma$-complex $Z$ such that the stabilizer of every cell is of type $F_{\infty}$. Let $\left\{Z_{q}\right\}_{q \in \mathbb{N}}$ be a filtration of $Z$ such that each $Z_{q}$ is finite mod $\Gamma$. Suppose that the connectivity of the pair $\left(Z_{q+1}, Z_{q}\right)$ tends to $\infty$ as $q$ tends to $\infty$. Then $\Gamma$ is of type $F_{\infty}$.

First, we need to modify the finite directed graph $(\mathcal{V}, \mathcal{E})$ so that its adjacency matrix $M$ has a certain special property. Let $N=(N(i, j))_{1 \leq i, j \leq n}$ be a matrix with entries in $\mathbb{N}$. We say that $N$ is in canonical form if the following conditions are satisfied.

- For any $i \neq j, N(i, j)=1$.

- For any $i, N(i, i) \geq 2$.

- There exists $j$ such that $N(j, j)=2$. 
Note that $N$ is primitive and $N=N^{t}$. Take $j$ such that $N(j, j)=2$. For $i \neq j$, let $d_{i}=N(i, i)-2$. It is not so hard to see

$$
\operatorname{Coker}\left(\mathrm{id}-N^{t}\right) \cong \bigoplus_{i \neq j} \mathbb{Z}_{d_{i}} \text { and } \operatorname{det}\left(\mathrm{id}-N^{t}\right)=(-1)^{n} \prod_{i \neq j} d_{i},
$$

where $\mathbb{Z}_{1}$ is understood as 0 and $\mathbb{Z}_{0}$ is understood as $\mathbb{Z}$. Hence, for the given matrix $M$, we can construct a matrix $N$ in canonical form satisfying

$$
\text { Coker }\left(\mathrm{id}-M^{t}\right) \cong \operatorname{Coker}\left(\mathrm{id}-N^{t}\right) \quad \text { and } \quad \operatorname{det}\left(\mathrm{id}-M^{t}\right)=\operatorname{det}\left(\mathrm{id}-N^{t}\right) .
$$

Let $(\tilde{X}, \tilde{\sigma})$ be the one-sided shift of finite type arising from the matrix $N$ and let $\tilde{G}$ be the étale groupoid of $(\tilde{X}, \tilde{\sigma})$. Then there exists an isomorphism $\varphi: H_{0}(G) \rightarrow H_{0}(\tilde{G})$. By Lemma 5.3 , there exists a non-empty clopen set $\tilde{Y} \subset \tilde{X}$ such that $\left[1_{\tilde{Y}}\right]_{\tilde{G}}=\varphi\left(\left[1_{Y}\right]_{G}\right)$. It follows from Theorem 6.2 that $G \mid Y$ is isomorphic to $\tilde{G} \mid \tilde{Y}$. Therefore, by replacing $G \mid Y$ with $\tilde{G} \mid \tilde{Y}$, we may assume that the adjacency matrix $M$ of the graph $(\mathcal{V}, \mathcal{E})$ is in canonical form. Set $\mathcal{V}_{0}=\left\{\zeta \in \mathcal{V} \mid M^{t}(\zeta, \zeta)=2\right\}$. Then

$$
a \in \operatorname{Ker}\left(\mathrm{id}-M^{t}\right) \Longleftrightarrow \sum_{\zeta \in \mathcal{V}_{0}} a(\zeta)=0 \quad \text { and } \quad a(\xi)=0 \quad \forall \xi \in \mathcal{V} \backslash \mathcal{V}_{0}
$$

This property will play an important role in the argument below.

We write $\mathbb{Z}_{+}=\mathbb{N} \cup\{0\}$. For an admissible word $\mu$, we define a compact open $G$-set $U_{\mu}$ by

$$
U_{\mu}=\left\{(x,|\mu|, y) \in G \mid \sigma^{|\mu|}(x)=y, x \in C_{\mu}\right\} .
$$

In other words, $U_{\mu}$ equals $U_{\mu, \emptyset}$, where $\emptyset$ denotes the empty word of length zero. For $\xi \in \mathcal{V}$, we let $i^{-1}(\xi)=\{e \in \mathcal{E} \mid i(e)=\xi\}$. Define $\hat{\xi} \in \mathbb{Z}_{+}^{\mathcal{V}}$ by

$$
\hat{\xi}(\eta)= \begin{cases}1 & \eta=\xi \\ 0 & \eta \neq \xi\end{cases}
$$

Recall that $D_{\xi}=\left\{\left(x_{n}\right)_{n} \in X \mid i\left(x_{1}\right)=\xi\right\}$.

Now we would like to construct a poset (partially ordered set) $\mathcal{B}$. Let $\mathcal{B}_{0}$ denote the set of all compact open $G$-sets $U$ such that $r(U) \subset Y$ and $s(U)=D_{\xi}$ for some $\xi \in \mathcal{V}$. For a finite subset $u$ of $\mathcal{B}_{0}$, we define $\operatorname{rank}(u) \in \mathbb{Z}_{+}^{\mathcal{V}}$ by

$$
\operatorname{rank}(u)(\xi)=\#\left\{U \in u \mid s(U)=D_{\xi}\right\} \quad \forall \xi \in \mathcal{V} .
$$

We let $\mathcal{B}$ denote the set of all finite subsets $u$ of $\mathcal{B}$ such that $\{r(U) \mid U \in u\}$ is a clopen partition of $Y$. We equip $\mathcal{B}$ with a partial order and view it as a poset in the following way. Given $u \in \mathcal{B}$ and an element $U \in u$ with $s(U)=D_{\xi}$, we get $v \in \mathcal{B}$ by replacing $U$ with the sets $U U_{e}$ for $e \in \mathcal{E}$ with $i(e)=\xi$. Thus,

$$
v=(u \backslash\{U\}) \cup\left\{U U_{e} \in \mathcal{B}_{0} \mid e \in i^{-1}(\xi)\right\} .
$$

One says that $v$ is a simple expansion of $u$. Notice that $\operatorname{rank}(v)$ is equal to $\operatorname{rank}(u)+$ $\left(M^{t}-\mathrm{id}\right) \hat{\xi}$. Conversely, if we start with $v \in \mathcal{B}, \xi \in \mathcal{V}$ and an injection $f: i^{-1}(\xi) \rightarrow v$ such that $s(f(e))=D_{t(e)}$, then we obtain $u \in \mathcal{B}$ by replacing the elements $f(e)$ with

$$
\bigcup_{i(e)=\xi} f(e) U_{e}^{-1} \in \mathcal{B}_{0} .
$$


The element $u$ is called a simple contraction of $v$. Again we have $\operatorname{rank}(u)=\operatorname{rank}(v)+$ $\left(\right.$ id $\left.-M^{t}\right) \hat{\xi}$. Given two elements $u, v \in \mathcal{B}, v$ is a simple expansion of $u$ if and only if $u$ is a simple contraction of $v$. We now iterate these construction. We say that $v \in \mathcal{B}$ is an expansion of $u \in \mathcal{B}$ and that $u$ is a contraction of $v$ if there exists a sequence $u=u_{0}, u_{1}, \ldots, u_{d}=v(d \geq 0)$ with $u_{i}$ a simple expansion of $u_{i-1}$ for $i=1,2, \ldots, d$. Then we equip $\mathcal{B}$ with a partial order by saying $u \leq v$ if $v$ is an expansion of $u$.

Lemma 6.13. The poset $\mathcal{B}$ is directed, i.e. for any $u, v \in \mathcal{B}$ there exists $w \in \mathcal{B}$ such that $u \leq w$ and $v \leq w$.

Proof. Let $u \in \mathcal{B}$. We first claim that there exists an expansion $w$ of $u$ such that any $W \in w$ is of the form $U_{\mu}$ for some admissible word $\mu$. Take $U \in u$ which is not of the form $U_{\mu}$. Let $s(U)=D_{\xi}$. There exist admissible words $\mu_{1}, \mu_{2}, \ldots, \mu_{k}, \nu_{1}, \nu_{2}, \ldots, \nu_{k}$ such that $U$ equals the disjoint union $\bigcup_{i=1}^{k} U_{\mu_{i}, \nu_{i}}$. Consider a simple expansion

$$
u^{\prime}=(u \backslash\{U\}) \cup\left\{U U_{e} \in \mathcal{B}_{0} \mid e \in i^{-1}(\xi)\right\}
$$

of $u$. Then for any $e \in i^{-1}(\xi)$,

$$
U U_{e}=\bigcup U_{\mu_{i}, \tilde{\nu}_{i}}
$$

where the union runs over all $i$ such that the first letter of $\nu_{i}$ is $e$, and $\tilde{\nu}_{i}$ is the (possibly empty) word obtained by removing the first letter of $\nu_{i}$. In particular, $\left|\tilde{\nu}_{i}\right|=\left|\nu_{i}\right|-1$. By repeating this argument, we finally obtain an expansion $w$ of $u$ for which every $\nu_{i}$ is of length zero. The proof of the claim is complete.

By the claim above, we may assume that there exist admissible words $\mu_{1}, \mu_{2}, \ldots, \mu_{k}$, $\nu_{1}, \nu_{2}, \ldots, \nu_{l}$ such that

$$
u=\left\{U_{\mu_{i}} \mid i=1,2, \ldots, k\right\} \quad \text { and } \quad v=\left\{U_{\nu_{j}} \mid j=1,2, \ldots, l\right\} .
$$

Let $m=\max \left\{\left|\mu_{i}\right|,\left|\nu_{j}\right| \mid i=1, \ldots, k, j=1, \ldots, l\right\}$. Suppose that $\left|\mu_{i}\right|$ is less than $m$. Then

$$
u^{\prime}=\left(u \backslash\left\{U_{\mu_{i}}\right\}\right) \cup\left\{U_{\mu_{i} e} \in \mathcal{B}_{0} \mid e \in \mathcal{E}, t\left(\mu_{i}\right)=i(e)\right\}
$$

is a simple expansion of $u$ and $\left|\mu_{i} e\right|=\left|\mu_{i}\right|+1$. By repeating this argument, we can find an expansion $\tilde{u}$ of $u$ such that any element of $\tilde{u}$ is of the form $U_{\mu}$ with $|\mu|=m$. In the same way, there exists an expansion $\tilde{v}$ of $v$ such that any element of $\tilde{v}$ is of the form $U_{\mu}$ with $|\mu|=m$. This means that $\tilde{u}$ equals $\tilde{v}$, which completes the proof.

We denote by $|\mathcal{B}|$ the simplicial complex whose simplices are the non-empty finite linearly ordered subsets of $\mathcal{B}$. For a subset $\mathcal{C} \subset \mathcal{B},|\mathcal{C}|$ denotes the subcomplex of $|\mathcal{B}|$ spanned by $\mathcal{C}$. We identify the set of vertices of $|\mathcal{B}|$ with $\mathcal{B}$.

We now define an action of $[[G \mid Y]]$ on $|\mathcal{B}|$. For $\alpha \in[[G \mid Y]]$, take a compact open $G \mid Y$-set $W$ such that $\alpha=\pi_{W}$. Note that $r(W)=Y$ and $s(W)=Y$. For $u \in \mathcal{B}$, we define

$$
\alpha u=\left\{W U \in \mathcal{B}_{0} \mid U \in u\right\} .
$$

This gives a well-defined action of $[[G \mid Y]]$ on $\mathcal{B}$. It is easy to see that $u \mapsto \alpha u$ is an order preserving map. Hence this action gives rise to an action of $[[G \mid Y]]$ on the simplicial complex $|\mathcal{B}|$. 
Lemma 6.14. (1) For $u, v \in \mathcal{B}$, there exists $\alpha \in[[G \mid Y]]$ such that $\alpha u=v$ if and only if $\operatorname{rank}(u)=\operatorname{rank}(v)$.

(2) Every vertex stabilizer is a finite group. More precisely, for any $u \in \mathcal{B},\{\alpha \in[[G \mid Y]] \mid$ $\alpha u=u\}$ is isomorphic to $\bigoplus_{\xi \in \mathcal{V}} \Sigma_{\operatorname{rank}(u)(\xi)}$, where $\Sigma_{n}$ stands for the symmetric group on $n$ letters.

Proof. (1) The 'only if' part is obvious. To prove the 'if' part, $\operatorname{assume} \operatorname{rank}(u)=\operatorname{rank}(v)$. There exists a bijection $f: u \rightarrow v$ such that $s(f(U))=s(U)$ for every $U \in u$. Then

$$
W=\bigcup_{U \in u} f(U) U^{-1}
$$

is a compact open $G \mid Y$-set satisfying $r(W)=s(W)=Y$ and $\pi_{W} u=v$.

(2) Assume $\alpha u=u$. Take a compact open $G \mid Y$-set $W$ such that $\alpha=\pi_{W}$. The homeomorphism $\alpha$ induces a permutation of the finite set $u$. Suppose that the induced permutation is the identity. For every $U \in u$, we have $W U=U$, and so

$$
W=W Y=\bigcup_{U \in u} W U U^{-1}=\bigcup_{U \in u} U U^{-1}=Y
$$

Thus $\alpha$ is the identity. It is clear that the induced permutations form a group isomorphic to $\bigoplus_{\xi} \Sigma_{\operatorname{rank}(u)(\xi)}$.

Let $\omega \in \mathcal{B}$. By replacing $\omega$ with its any simple expansion, we may assume that $\operatorname{rank}(\omega)(\xi)$ is positive for any $\xi \in \mathcal{V}$, because each entry of $M^{t}$ - id is positive. We fix such $\omega \in \mathcal{B}$ and set

$$
\mathcal{B}_{\omega}=\{u \in \mathcal{B} \mid \alpha \omega \leq u \text { for some } \alpha \in[[G \mid Y]]\} .
$$

Since each entry of $\operatorname{rank}(\omega)$ and $M^{t}$-id is positive, from Lemma 6.14 (1) one can see that $B_{\omega}$ is equal to

$$
\left\{u \in \mathcal{B} \mid \operatorname{rank}(u)=\operatorname{rank}(\omega)+\left(M^{t}-\mathrm{id}\right) a \text { for some } a \in \mathbb{Z}_{+}^{\mathcal{V}}\right\} .
$$

As $\mathcal{B}$ is directed, so is $\mathcal{B}_{\omega}$. This readily implies the following (see [15, Proposition 9.3.14] for instance).

Lemma 6.15. The simplicial complex $\left|\mathcal{B}_{\omega}\right|$ is contractible.

For $u \in \mathcal{B}_{\omega}$, choose $a \in \mathbb{Z}_{+}^{\mathcal{V}}$ so that

$$
\operatorname{rank}(u)=\operatorname{rank}(\omega)+\left(M^{t}-\mathrm{id}\right) a .
$$

Put

$$
\operatorname{height}(u)=\sum_{\xi \in \mathcal{V}} a(\xi)
$$

Thanks to (6.4), height $(u)$ does not depend on the choice of $a$. If $v$ is a simple expansion of $u \in \mathcal{B}_{\omega}$, then height $(v)=$ height $(u)+1$. Clearly height $(u)$ equals the largest integer $n$ such that there exists a linearly ordered $(n+1)$-tuple $u_{0}<u_{1}<\cdots<u_{n}=u$ in $\mathcal{B}_{\omega}$. Because each entry of $M^{t}-$ id is positive, one has $\operatorname{rank}(u)(\xi) \geq \operatorname{height}(u)$ for every $\xi \in \mathcal{V}$. We also note that the $[[G \mid Y]]$-action preserves height, i.e. height $(\alpha u)=\operatorname{height}(u)$. 
Next, we would like to construct a contractible $[[G \mid Y]]$-invariant subcomplex $Z \subset\left|\mathcal{B}_{\omega}\right|$. Given an element $u \in \mathcal{B}$, an elementary expansion of $u$ is an element $v \in \mathcal{B}$ obtained by choosing a subset $u^{\prime} \subset u$ and taking simple expansions of $u$ at each of $U \in u^{\prime}$, that is,

$$
v=\left(u \backslash u^{\prime}\right) \cup\left\{U U_{e} \in \mathcal{B}_{0} \mid U \in u^{\prime}, s(U)=D_{\xi}, e \in i^{-1}(\xi)\right\} .
$$

We call a simplex $u_{0}<u_{1}<\cdots<u_{n}$ of $\left|\mathcal{B}_{\omega}\right|$ elementary if $u_{n}$ is an elementary expansion of $u_{0}$. This implies that $u_{j}$ is an elementary expansion of $u_{i}$ for any $i \leq j$. Hence any face of an elementary simplex is elementary. Clearly elementary simplices are preserved by the action of $[[G \mid Y]]$. It follows that the union $Z$ of all elementary simplices is a $[[G \mid Y]]$-invariant subcomplex of $\left|\mathcal{B}_{\omega}\right|$.

In what follows, we will use the standard notation for intervals in a poset. For example, the open interval $(u, v)$ is defined by $(u, v)=\left\{w \in \mathcal{B}_{\omega} \mid u<w<v\right\}$. The closed interval $[u, v]$ and the half-open intervals $[u, v)$ and $(u, v]$ are defined similarly. The following is exactly the same as the lemma of [5, Section 4].

Lemma 6.16. Let $v \in \mathcal{B}_{\omega}$ be an expansion of $u \in \mathcal{B}_{\omega}$. If $v$ is not an elementary expansion of $u$, then the subcomplex $|(u, v)|$ is contractible.

The following is exactly the same as [5, Theorem 1]. We include the argument here for completeness.

Lemma 6.17. The complex $Z$ is contractible.

Proof. Let $W_{n}$ be the complex obtained by adjoining to $Z$ the subcomplexes of the form $|[u, v]|$ with $v$ a non-elementary expansion of $u \in \mathcal{B}_{\omega}$ and $\operatorname{height}(v)-\operatorname{height}(u) \leq n$. Since $\left|\mathcal{B}_{\omega}\right|$ is contractible by Lemma 6.15 and $\left|\mathcal{B}_{\omega}\right|$ equals the union $\bigcup_{n} W_{n}$, it suffices to show that the inclusion $Z \hookrightarrow W_{n}$ is a homotopy equivalence. Suppose by induction that $Z \hookrightarrow W_{n-1}$ is a homotopy equivalence. Let $v$ be a non-elementary expansion of $u \in \mathcal{B}_{\omega}$ $\operatorname{such}$ that height $(v)-\operatorname{height}(u)=n$. The intersection of $|[u, v]|$ with $W_{n-1}$ is $|[u, v) \cup(u, v]|$, which is the suspension of $|(u, v)|$ and hence is contractible by the lemma above. Since $|[u, v]|$ is also contractible for trivial reasons, the adjunction of $|[u, v]|$ does not change the homotopy type of $W_{n-1}$. Two such subcomplexes $|[u, v]|$ intersect only in $W_{n-1}$, and so the inclusion $W_{n-1} \hookrightarrow W_{n}$ is a homotopy equivalence. The proof is complete.

We remark that the argument above goes through without change if we fix $p \in \mathbb{Z}_{+}$ and replace $\mathcal{B}_{\omega}$ with $\left\{u \in \mathcal{B}_{\omega} \mid\right.$ height $\left.(u) \geq p\right\}$. Therefore the subcomplex of $Z$ spanned by the vertices $u$ with height $(u) \geq p$ is also contractible.

For every $u \in \mathcal{B}_{\omega}$, we introduce the (finite) simplicial complex $K_{u}$ as follows. Consider a pair $(\xi, f)$ of $\xi \in \mathcal{V}$ and an injection $f: i^{-1}(\xi) \rightarrow u$ such that $s(f(e))=D_{t(e)}$. A simplex of $K_{u}$ is a finite collection $\left\{\left(\xi_{0}, f_{0}\right), \ldots,\left(\xi_{m}, f_{m}\right)\right\}$ of such pairs satisfying the following conditions.

- The images of the maps $f_{j}$ are pairwise disjoint.

- There exists $a \in \mathbb{Z}_{+}^{\mathcal{V}}$ such that

$$
\operatorname{rank}(u)-\left(M^{t}-\mathrm{id}\right)\left(\hat{\xi}_{0}+\cdots+\hat{\xi}_{m}\right)=\operatorname{rank}(\omega)+\left(M^{t}-\mathrm{id}\right) a .
$$


The first condition says that by contracting $\operatorname{Im} f_{j}$ we can obtain $v$ such that $u$ is an elementary expansion of $v$. The second condition says that $v$ is still in $\mathcal{B}_{\omega}$, because the left-hand side of the equality is the rank of $v$.

Lemma 6.18. For any integer $n \geq 0$, there exists $r \in \mathbb{N}$ such that the simplicial complex $K_{u}$ is n-connected provided height $(u)>r$.

Proof. Let $l=\max \{M(\xi, \eta) \mid \xi, \eta \in \mathcal{V}\}$. We claim that for any $m \in \mathbb{Z}_{+}$and $k \in \mathbb{N}$ the following holds: if

$$
\operatorname{height}(u)>\max \{(m+1) \# \mathcal{V}, k(m+1) l+l\}
$$

and $P_{1}, \ldots, P_{k}$ are $m$-simplices of $K_{u}$, then we can find a vertex $(\eta, g)$ of $K_{u}$ such that $P_{j} \cup\{(\eta, g)\}$ is an $(m+1)$-simplex of $K_{u}$ for any $j$. Choose $a \in \mathbb{Z}_{+}^{\mathcal{V}}$ so that $\operatorname{rank}(u)=$ $\operatorname{rank}(\omega)+\left(M^{t}-\mathrm{id}\right) a$. From

$$
\sum_{\xi \in \mathcal{V}} a(\xi)=\operatorname{height}(u)>(m+1) \# \mathcal{V}
$$

we can find $\eta \in \mathcal{V}$ such that $a(\eta)>m+1$. Let

$$
u^{\prime}=\bigcup_{j=1}^{k} \bigcup_{(\xi, f) \in P_{j}} \operatorname{Im} f .
$$

Then for any $\xi \in \mathcal{V}$ we have

$$
\#\left\{U \in u \backslash u^{\prime} \mid s(U)=D_{\xi}\right\} \geq \operatorname{rank}(u)(\xi)-k(m+1) l \geq \operatorname{height}(u)-k(m+1) l>l .
$$

Hence we can find an injection $g: i^{-1}(\eta) \rightarrow u$ such that $s(g(e))=D_{t(e)}$ for all $e \in i^{-1}(\eta)$ and $u^{\prime} \cap \operatorname{Im} g=\emptyset$. Now for each $j=1,2, \ldots, k$, let

$$
b_{j}=a-\sum_{(\xi, f) \in P_{j}} \hat{\xi}
$$

so that

$$
\operatorname{rank}(u)-\sum_{(\xi, f) \in P_{j}}\left(M^{t}-\mathrm{id}\right) \hat{\xi}=\operatorname{rank}(\omega)+\left(M^{t}-\mathrm{id}\right) b_{j} .
$$

Since $P_{j}$ is a simplex of $K_{u}$, by (6.4), one has

$$
\sum_{\zeta \in \mathcal{V}_{0}} b_{j}(\zeta) \geq 0 \quad \text { and } \quad b_{j}(\xi) \geq 0 \quad \forall \xi \in \mathcal{V} \backslash \mathcal{V}_{0}
$$

From the choice of $\eta$, we can verify

$$
\sum_{\zeta \in \mathcal{V}_{0}}\left(b_{j}-\hat{\eta}\right)(\zeta) \geq 0 \quad \text { and } \quad\left(b_{j}-\hat{\eta}\right)(\xi) \geq 0 \quad \forall \xi \in \mathcal{V} \backslash \mathcal{V}_{0}
$$

From (6.4), we can conclude that $P_{j} \cup\{(\eta, g)\}$ is an $(m+1)$-simplex of $K_{u}$ for any $j$.

Once this claim is established, the rest of the proof proceeds in the same manner as [5. Lemma 4.20]. We omit the details. 
Notice that the natural number $r$ obtained in the lemma above depends only on the adjacency matrix $M$ and does not depend on the choice of $\omega \in \mathcal{B}$.

Given integers $p, q$ with $0 \leq p \leq q$, we let $Z_{p, q}$ be the subcomplex of $Z$ spanned by the vertices $u$ with $p \leq$ height $(u) \leq q$. Note that $Z_{p, q}$ is $[[G \mid Y]]$-invariant. Note also that the dimension of $Z_{p, q}$ is at most $q-p$. The following is almost the same as [5, Theorem 2]. We include the proof for completeness.

Lemma 6.19. For any integer $n \geq 0$, there exists $r \in \mathbb{N}$ such that the inclusion $Z_{p, q} \hookrightarrow$ $Z_{p, q+1}$ induces isomorphisms $\pi_{i}\left(Z_{p, q}\right) \rightarrow \pi_{i}\left(Z_{p, q+1}\right)$ for all $i \leq n$, provided $q \geq r$ and $q \geq p+n+1$. In particular, for such $p, q$. $Z_{p, q}$ is $n$-connected.

Proof. For given $n \geq 0$, let $r \in \mathbb{N}$ be as in the lemma above. The inclusion $Z_{p, q} \hookrightarrow Z_{p, q+1}$ is obtained by adjoining, for each $u \in \mathcal{B}_{\omega}$ with height $(u)=q+1$, a cone over the link $L$ of $u$ in $Z_{p, q}$. The vertices of this link $L$ are $v \in \mathcal{B}_{\omega}$ such that $u$ is an elementary expansion of $v$ and $p \leq$ height $(v) \leq q$, and the simplices of $L$ are linearly ordered tuples $v_{0}<v_{1}<\cdots<v_{m}$.

We can describe a vertex $v$ of $L$ by specifying which elements of $u$ are contracted to get $v$. More precisely, each vertex $v$ of $L$ corresponds to a set $P=\left\{\left(\xi_{0}, f_{0}\right), \ldots,\left(\xi_{m}, f_{m}\right)\right\}$ consisting of pairs of $\xi_{j} \in \mathcal{V}$ and injections $f_{j}: i^{-1}\left(\xi_{j}\right) \rightarrow u$ such that the following conditions are satisfied.

- $s\left(f_{j}(e)\right)=D_{t(e)}$ for every $e \in i^{-1}\left(\xi_{j}\right)$.

- $\operatorname{Im} f_{j}$ are pairwise disjoint.

- There exists $a \in \mathbb{Z}_{+}^{\mathcal{V}}$ such that

$$
\operatorname{rank}(u)-\left(M^{t}-\mathrm{id}\right)\left(\hat{\xi}_{0}+\cdots+\hat{\xi}_{m}\right)=\operatorname{rank}(\omega)+\left(M^{t}-\mathrm{id}\right) a .
$$

- $q-m \geq p$.

The simplices of $L$ correspond to chains $P_{0} \subset \cdots \subset P_{l}$ of such sets $P$. Now such $P$ are the same as simplices of the complex $K_{u}$ defined above that have dimension at most $q-p$. It follows that the complex $L$ is the barycentric subdivision of the $(q-p)$-skeleton of $K_{u}$. The lemma above implies that $K_{u}$ is $n$-connected, because height $(u)=q+1>r$. Hence $L$ is $n$-connected, too. Attaching a cone over such a complex $L \subset Z_{p, q}$ does not affect $\pi_{i}$ for $i \leq n$. Consequently, $\pi_{i}\left(Z_{p, q}\right) \rightarrow \pi_{i}\left(Z_{p, q+1}\right)$ is an isomorphism as required.

Consider the sequence of inclusions $Z_{p, q} \subset Z_{p, q+1} \subset Z_{p, q+2} \subset \ldots$ The union is the subcomplex of $Z$ spanned by the vertices $u$ with height $(u) \geq p$. This union is contractible by Lemma 6.17 and the remark following it. Therefore $Z_{p, q}$ is $n$-connected.

Lemma 6.20. For any integers $p, q$ with $0 \leq p \leq q$, the simplicial complex $Z_{p, q}$ is finite $\bmod [[G \mid Y]]$.

Proof. Let $u_{0}<u_{1}<\cdots<u_{m}$ be an elementary simplex. For every $j=1,2, \ldots, m$, let $\tilde{u}_{j} \subset u_{0}$ be the subset such that $u_{j}$ is obtained by taking simple expansions of $u_{0}$ at each of $U \in u_{0} \backslash \tilde{u}_{j}$. Then we have

$$
u_{0} \supseteq \tilde{u}_{1} \supseteq \tilde{u}_{2} \supseteq \cdots \supseteq \tilde{u}_{m} .
$$


Let $v_{0}<v_{1}<\cdots<v_{m}$ be another elementary simplex. Define $\tilde{v}_{1}, \tilde{v}_{2}, \ldots, \tilde{v}_{m}$ in the same way. We claim that if $\operatorname{rank}\left(u_{0}\right)=\operatorname{rank}\left(v_{0}\right)$ and $\operatorname{rank}\left(\tilde{u}_{j}\right)=\operatorname{rank}\left(\tilde{v}_{j}\right)$ for every $j$, then there exists $\alpha \in[[G \mid Y]]$ such that $\alpha u_{j}=v_{j}$ for all $j=0,1, \ldots, m$. From $\operatorname{rank}\left(\tilde{u}_{m}\right)=\operatorname{rank}\left(\tilde{v}_{m}\right)$, we can find a bijection $f: \tilde{u}_{m} \rightarrow \tilde{v}_{m}$ satisfying $s(U)=s(f(U))$ for all $U \in \tilde{u}_{m}$. Using $\operatorname{rank}\left(\tilde{u}_{m} \backslash \tilde{u}_{m-1}\right)=\operatorname{rank}\left(\tilde{v}_{m} \backslash \tilde{v}_{m-1}\right)$, we can extend $f$ to a bijection from $\tilde{u}_{m-1}$ to $\tilde{v}_{m-1}$ satisfying $s(U)=s(f(U))$ for all $U \in \tilde{u}_{m-1}$. Repeating this argument, we finally obtain a bijection $f: u_{0} \rightarrow v_{0}$ such that $f\left(\tilde{u}_{j}\right)=\tilde{v}_{j}$ for any $j$ and $s(U)=s(f(U))$ for any $U \in u_{0}$. Define a compact open $G \mid Y$-set $W$ by

$$
W=\bigcup_{U \in u_{0}} f(U) U^{-1}
$$

Then $\alpha=\pi_{W} \in[[G \mid Y]]$ satisfies $\alpha u_{j}=v_{j}$ for all $j=0,1, \ldots, m$.

If $u_{0}<u_{1}<\cdots<u_{m}$ is a simplex of $Z_{p, q}$, then height $\left(u_{0}\right)$ is at most $q-m$. Evidently $\left\{\operatorname{rank}(u) \in \mathbb{Z}^{\mathcal{V}} \mid \operatorname{height}(u) \leq q-m\right\}$ is finite. Hence the number of $(m+1)$-tuples $\left(\operatorname{rank}\left(u_{0}\right), \operatorname{rank}\left(\tilde{u}_{1}\right), \ldots, \operatorname{rank}\left(\tilde{u}_{m}\right)\right)$ for $m$-simplices of $Z_{p, q}$ is finite. It follows from the claim above that $m$-simplices of $Z_{p, q}$ is finite $\bmod [[G \mid Y]]$.

We are now in a position to apply Brown's theorem.

Theorem 6.21. The group $[[G \mid Y]]$ is of type $F_{\infty}$. In other words, the group $[[G \mid Y]]$ is finitely presented and is of type $F P_{\infty}$.

Proof. This immediately follows from Lemma 6.14 (2), Lemma 6.19, Lemma 6.20 and Theorem 6.12,

\subsection{A presentation for $[[G \mid Y]]$}

As in Section 6.1, we let $G$ be the étale groupoid arising from a one-sided irreducible shift of finite type $(X, \sigma)$. We use the notation of Section 6.1. Fix a non-empty clopen subset $Y \subset X$. In this subsection, we would like to describe generators and relations for $[[G \mid Y]]$. This enables us to calculate the abelianization of $[[G \mid Y]]$ (Corollary 6.24). Consequently, the commutator subgroup $D([[G \mid Y]])$ is shown to be type $\mathrm{F}_{\infty}$ when $H_{0}(G)$ is a finite abelian group (Corollary 6.25).

As in the last subsection, we assume that the adjacency matrix $M$ is in canonical form, and put $\mathcal{V}_{0}=\left\{\eta \in \mathcal{V} \mid M^{t}(\eta, \eta)=2\right\}$. Fix an element $\zeta \in \mathcal{V}_{0}$. Set $\mathcal{V}_{1}=\left(\mathcal{V} \backslash \mathcal{V}_{0}\right) \cup\{\zeta\}$. Notice that the rank of the free abelian group $H_{1}(G)$ is equal to $\#\left(\mathcal{V} \backslash \mathcal{V}_{1}\right)=\#\left(\mathcal{V}_{0} \backslash\{\zeta\}\right)$. We equip $\mathcal{V} \backslash \mathcal{V}_{1}$ with a linear order. We regard $\mathbb{Z}^{\mathcal{V}_{1}}$ as a subgroup of $\mathbb{Z}^{\mathcal{V}}$. By (6.4), id $-M^{t}$ is injective on $\mathbb{Z}^{\mathcal{V}_{1}}$, i.e. for $a \in \mathbb{Z}^{\mathcal{V}_{1}}$, (id $\left.-M^{t}\right) a=0$ implies $a=0$. For $a \in \mathbb{Z}_{+}^{\mathcal{V}}$, we define $\|a\|=\sum_{\xi \in \mathcal{V}} a(\xi)$.

In the previous subsection, we constructed contractible $[[G \mid Y]]$-invariant complexes $Z \subset\left|\mathcal{B}_{\omega}\right|$. For $p<q, Z_{p, q}$ denotes the subcomplex of $Z$ spanned by vertices $u$ with $p \leq$ height $(u) \leq q$. In Lemma 6.19, we proved that $Z_{p, q}$ is $n$-connected if $p$ is large enough and $q-p>n$. In particular, there exists $p \in \mathbb{N}$ such that the 2-dimensional simplicial complex $Z_{p, p+2}$ is 1-connected. We would like to examine the $[[G \mid Y]]$-action on $Z_{p, p+2}$ and write down generators and relations of $[[G \mid Y]]$, by using [3, Theorem 1].

As pointed out in the remark following Lemma 6.18, the natural number $p$ depends only on the matrix $M$ and does not depend on the choice of $\omega \in \mathbb{Z}_{+}^{\mathcal{V}}$. Hence, by replacing 
$\omega$ with its certain expansion if necessary, we may assume $\operatorname{rank}(\omega)(\xi) \geq p+2$ for any $\xi \in \mathcal{V}$. Let

$$
f: \mathcal{V} \times\{1,2, \ldots, p+2\} \rightarrow \omega
$$

be an injection satisfying $s(f(\xi, j))=D_{\xi}$ for every $(\xi, j)$. For each $a \in \mathbb{Z}_{+}^{\mathcal{V}}$ with $\|a\| \leq p+2$, we let

$$
u=\{f(\xi, j) \in \omega \mid \xi \in \mathcal{V}, 1 \leq j \leq a(\xi)\}
$$

and define a vertex $\omega(a)$ of $Z$ by

$$
\omega(a)=(\omega \backslash u) \cup\left\{U U_{e} \mid U \in u, s(U)=D_{\xi}, e \in i^{-1}(\xi)\right\} .
$$

Thus, $\omega(a)$ is the elementary expansion of $\omega$ with respect to the subset $u \subset \omega$. Clearly $\operatorname{rank}(\omega(a))=\operatorname{rank}(\omega)+\left(M^{t}-\mathrm{id}\right) a$, and height $(\omega(a))=\|a\|$. Note also that $\omega\left(a^{\prime}\right)$ is an elementary expansion of $\omega(a)$ if $a(\xi) \leq a^{\prime}(\xi)$ for every $\xi \in \mathcal{V}$.

For every $\eta \in \mathcal{V} \backslash \mathcal{V}_{1}$, we associate an element $\theta_{\eta} \in[[G \mid Y]]$ as follows. First, for each $\eta \in \mathcal{V}_{0}$, we choose an edge $e_{\eta} \in \mathcal{E}$ such that $i\left(e_{\eta}\right)=t\left(e_{\eta}\right)=\eta$. Let $\eta \in \mathcal{V} \backslash \mathcal{V}_{1}$. Since the matrix $M$ is in canonical form, there exists a unique bijection

$$
h: i^{-1}(\zeta) \backslash\left\{e_{\zeta}\right\} \rightarrow i^{-1}(\eta) \backslash\left\{e_{\eta}\right\}
$$

satisfying $t(h(e))=t(e)$. Let $U=f(\zeta, 1)$ and $V=f(\eta, 1)$. Define a compact open $G \mid Y$-set $W$ by

$$
W=U\left(U U_{e_{\zeta}}\right)^{-1} \cup\left(V U_{e_{\eta}}\right) V^{-1} \cup \bigcup_{e} V U_{h(e), e} U^{-1} .
$$

One has $r(W)=s(W)=r(U) \cup r(V)$. Let $\tilde{W}=W \cup(Y \backslash r(W))$ and define $\theta_{\eta} \in[[G \mid Y]]$ by $\theta_{\eta}=\pi_{\tilde{W}}$. Then we have $\theta_{\eta} \omega(\hat{\zeta})=\omega(\hat{\eta})$. For every $\eta \in \mathcal{V}_{0}$ and $1 \leq k \leq p+2$, we define a compact open $G \mid Y$-set $U$ by

$$
U=f(\eta, 1) f(\eta, k)^{-1} \cup f(\eta, k) f(\eta, 1)^{-1}
$$

and put $\beta_{\eta, k}=\pi_{\tilde{U}} \in[[G \mid Y]]$, where $\tilde{U}=U \cup(Y \backslash r(U))$. It is easy to see that $\beta_{\eta, k} \omega=\omega$ and $\beta_{\eta, k}^{2}=1$. Moreover, for $\eta \in \mathcal{V} \backslash \mathcal{V}_{1}$ we have

$$
\left(\beta_{\zeta, k} \beta_{\eta, l} \theta_{\eta} \beta_{\eta, l} \beta_{\zeta, k}\right) \omega(a+\hat{\zeta})=\omega(a+\hat{\eta})
$$

if $a(\zeta)=k-1$ and $a(\eta)=l-1$.

For each $i=0,1,2$, we would like to find a set of representatives for the $i$-simplices of $Z_{p, p+2} \bmod [[G \mid Y]]$. For $p \leq k \leq p+2$, set

$$
A_{k}=\left\{a \in \mathbb{Z}_{+}^{\mathcal{V}_{1}} \mid\|a\|=k\right\} .
$$

Then, by Lemma 6.14 (1),

$$
C_{0}=\left\{\omega(a) \in Z_{p, p+2} \mid a \in A_{p} \cup A_{p+1} \cup A_{p+2}\right\}
$$

is a set of representatives for the vertices of $Z_{p, p+2} \bmod [[G \mid Y]]$, that is, for any vertex $u$ of $Z_{p, p+2}$ there exists a unique vertex $v \in C_{0}$ such that $\alpha u=v$ for some $\alpha \in[[G \mid Y]]$. 
We let $C_{1}$ be the set of all 1-simplices of the simplicial complex $\left|C_{0}\right|$. In other words,

$$
\begin{aligned}
C_{1}= & \left\{\omega(a)<\omega(a+\hat{\xi}) \mid a \in A_{p} \cup A_{p+1}, \quad \xi \in \mathcal{V}_{1}\right\} \\
& \cup\left\{\omega(a)<\omega\left(a+\hat{\xi}_{1}+\hat{\xi}_{2}\right) \mid a \in A_{p}, \quad \xi_{1}, \xi_{2} \in \mathcal{V}_{1}\right\} .
\end{aligned}
$$

In order to obtain a set of representatives for the 1-simplices mod $[[G \mid Y]]$, we consider the following 1-simplices.

(i) $\omega(a)<\omega(a+\hat{\eta})$ for $a \in A_{p}$ and $\eta \in \mathcal{V} \backslash \mathcal{V}_{1}$

(ii) $\omega(a)<\omega(a+\hat{\eta})$ for $a \in A_{p+1}$ and $\eta \in \mathcal{V} \backslash \mathcal{V}_{1}$.

(iii) $\omega(a)<\omega(a+\hat{\xi}+\hat{\eta})$ for $a \in A_{p}, \xi \in \mathcal{V}_{1}$ and $\eta \in \mathcal{V} \backslash \mathcal{V}_{1}$

(iv) $\omega(a)<\omega\left(a+\hat{\eta}_{1}+\hat{\eta}_{2}\right)$ for $a \in A_{p}$ and $\eta_{1}, \eta_{2} \in \mathcal{V} \backslash \mathcal{V}_{1}$

Let $C_{1}^{\prime}$ be the set of these 1 -simplices. Then $C_{1} \cup C_{1}^{\prime}$ is a set of representatives for the 1-simplices of $Z_{p, p+2} \bmod [[G \mid Y]]$,

Likewise, we let $C_{2}$ be the set of all 2-simplices of $\left|C_{0}\right|$, i.e.

$$
C_{2}=\left\{\omega(a)<\omega\left(a+\hat{\xi}_{1}\right)<\omega\left(a+\hat{\xi}_{1}+\hat{\xi}_{2}\right) \mid a \in A_{p}, \xi_{1}, \xi_{2} \in \mathcal{V}_{1}\right\}
$$

In order to obtain a set of representatives for the 2-simplices mod $[[G \mid Y]]$, we consider the following 2-simplices.

(v) $\omega(a)<\omega(a+\hat{\xi})<\omega(a+\hat{\xi}+\hat{\eta})$ for $a \in A_{p}, \xi \in \mathcal{V}_{1}$ and $\eta \in \mathcal{V} \backslash \mathcal{V}_{1}$

(vi) $\omega(a)<\omega(a+\hat{\eta})<\omega(a+\hat{\xi}+\hat{\eta})$ for $a \in A_{p}, \xi \in \mathcal{V}_{1}$ and $\eta \in \mathcal{V} \backslash \mathcal{V}_{1}$.

(vii) $\omega(a)<\omega\left(a+\hat{\eta}_{1}\right)<\omega\left(a+\hat{\eta}_{1}+\hat{\eta}_{2}\right)$ for $a \in A_{p}$ and $\eta_{1}, \eta_{2} \in \mathcal{V} \backslash \mathcal{V}_{1}$.

Let $C_{2}^{\prime}$ be the set of these 2-simplices. Then $C_{2} \cup C_{2}^{\prime}$ is a set of representatives for the 2-simplices of $Z_{p, p+2} \bmod [[G \mid Y]]$,

For each vertex $u$ of $Z_{p, p+2}$, let $\Sigma(u)$ denote the stabilizer of $u$. By Lemma 6.14 (2), $\Sigma(u)$ is naturally identified with a subgroup of the symmetric group on $u$ and is isomorphic to $\bigoplus_{\xi \in \mathcal{V}} \Sigma_{\operatorname{rank}(u)(\xi)}$. Similarly, for each 1-simplex $e$ of $Z_{p, p+2}$, we denote the stabilizer of $e$ by $\Sigma(e)$. We define an abstract group $\Gamma$ to be the free product of the groups $\Sigma(u)$ for $u \in C_{0}$ and a free group generated by elements $g_{e}$ for $e \in C_{1} \cup C_{1}^{\prime}$. It is clear that the group $\Gamma$ is finitely presented.

Next, we would like to introduce a surjective homomorphism $\pi: \Gamma \rightarrow[[G \mid Y]]$. To this end, for each $e=\{u<v\} \in C_{1} \cup C_{1}^{\prime}$, we choose $\gamma_{e} \in[[G \mid Y]]$ such that $\gamma_{e}^{-1}(v) \in C_{0}$ as follows. First, for $e \in C_{1}$, we put $\gamma_{e}=1$. Let us consider $\gamma_{e}$ for $e \in C_{1}^{\prime}$. For $e=\{\omega(a)<\omega(a+\hat{\eta})\}$ as in (i) and (ii), we put

$$
\gamma_{e}=\beta_{\zeta, k} \theta_{\eta} \beta_{\zeta, k},
$$

where $k=a(\zeta)+1$. For $e=\{\omega(a)<\omega(a+\hat{\xi}+\hat{\eta})\}$ as in (iii), we put

$$
\gamma_{e}= \begin{cases}\beta_{\zeta, k} \theta_{\eta} \beta_{\zeta, k} & \xi \neq \zeta \\ \beta_{\zeta, k+1} \theta_{\eta} \beta_{\zeta, k+1} & \xi=\zeta\end{cases}
$$


where $k=a(\zeta)+1$. For $e=\left\{\omega(a)<\omega\left(a+\hat{\eta}_{1}+\hat{\eta}_{2}\right)\right\}$ as in (iv), we put

$$
\gamma_{e}= \begin{cases}\left(\beta_{\zeta, k} \theta_{\eta_{1}} \beta_{\zeta, k}\right)\left(\beta_{\zeta, k+1} \theta_{\eta_{2}} \beta_{\zeta, k+1}\right) & \eta_{1}<\eta_{2} \\ \left(\beta_{\zeta, k} \theta_{\eta_{1}} \beta_{\zeta, k}\right)\left(\beta_{\zeta, k+1} \beta_{\eta_{2}, 2} \theta_{\eta_{2}} \beta_{\eta_{2} .2} \beta_{\zeta, k+1}\right) & \eta_{1}=\eta_{2},\end{cases}
$$

where $k=a(\zeta)+1$. Now we set $\pi\left(g_{e}\right)=\gamma_{e}$ for $e \in C_{1} \cup C_{1}^{\prime}$. This, together with the canonical inclusions $\Sigma(u) \rightarrow[[G \mid Y]]$, gives rise to a homomorphism $\pi: \Gamma \rightarrow[[G \mid Y]]$.

The following theorem says that the kernel of $\pi: \Gamma \rightarrow[[G \mid Y]]$ is finitely generated as a normal subgroup of $\Gamma$. In order to describe generators of the kernel, we need to introduce a little more notations. The 1-skeleton of $\left|C_{0}\right|$ is $C_{0} \cup C_{1}$, which can be regarded as a graph. Consider a maximal tree of this graph. Every vertex of $C_{0}$ is contained in the tree. Let us identify this tree with the corresponding subset $T \subset C_{1}$. For $e=\{u<v\} \in C_{1} \cup C_{1}^{\prime}$, we let $i_{e}: \Sigma(e) \rightarrow \Sigma(u)$ be the canonical inclusion and let $c_{e}: \Sigma(e) \rightarrow \Sigma(\tilde{v})$ be the homomorphism given by $\alpha \mapsto \gamma_{e}^{-1} \alpha \gamma_{e}$, where $\tilde{v}=\gamma_{e}^{-1} v \in C_{0}$. For each 2-simplex $\tau \in C_{2} \cup C_{2}^{\prime}$, as discussed in [3. Section 1], we can associate a 'relator' $r_{\tau} \in \Gamma$ by thinking of the boundary of $\tau$ as a closed path.

Theorem 6.22 ([3, Theorem 1]). The homomorphism $\pi: \Gamma \rightarrow[[G \mid Y]]$ is surjective and its kernel is generated by the following elements as a normal subgroup.

(1) $g_{e}$ for $e \in T$.

(2) $g_{e}^{-1} i_{e}(\alpha) g_{e} c_{e}\left(\alpha^{-1}\right)$ for $e \in C_{1} \cup C_{1}^{\prime}$ and $\alpha \in \Sigma(e)$.

(3) $r_{\tau}$ for $\tau \in C_{2} \cup C_{2}^{\prime}$.

The presentation given in the theorem above contains much redundancy. We would like to obtain a 'smaller' presentation of $[[G \mid Y]]$. First, let us look at $e \in C_{1} \backslash T$. Since $T$ is a maximal tree of the 1-skeleton of $\left|C_{0}\right|$, there exists a loop in $T \cup\{e\}$ containing $e$. Then, from $\pi\left(r_{\tau}\right)=1$ for all $\tau \in C_{2}$, we obtain

(4) $\pi\left(g_{e}\right)=1$ for all $e \in C_{1} \backslash T$.

Let us now look at the elements $r_{\tau} \in \Gamma$ associated with $\tau \in C_{2}^{\prime}$. From the 2-simplices $\tau=\{\omega(a)<\omega(a+\hat{\xi})<\omega(a+\hat{\xi}+\hat{\eta})\}$ as in (v), we get

(5) $\pi\left(g_{e}\right)=\pi\left(g_{e^{\prime}}\right)$ for all $e=\{\omega(a+\hat{\xi})<\omega(a+\hat{\xi}+\hat{\eta})\}$ and $e^{\prime}=\{\omega(a)<\omega(a+\hat{\xi}+\hat{\eta})\}$ with $a \in A_{p}, \xi \in \mathcal{V}_{1}$ and $\eta \in \mathcal{V} \backslash \mathcal{V}_{1}$

Next, let $\tau=\{\omega(a)<\omega(a+\hat{\eta})<\omega(a+\hat{\xi}+\hat{\eta})\}$ be a 2 -simplex as in (vi). In case $\xi \neq \zeta$, we obtain

(6) $\pi\left(g_{e}\right)=\pi\left(g_{e^{\prime}}\right)$ for all $e=\{\omega(a)<\omega(a+\hat{\eta})\}$ and $e^{\prime}=\{\omega(a)<\omega(a+\hat{\xi}+\hat{\eta})\}$ with $a \in A_{p}$, $\xi \in \mathcal{V}_{1} \backslash\{\zeta\}$ and $\eta \in \mathcal{V} \backslash \mathcal{V}_{1}$

In case $\xi=\zeta$, we have

(7) $\pi\left(\beta g_{e} \beta\right)=\pi\left(g_{e^{\prime}}\right)$ for all $e=\{\omega(a)<\omega(a+\hat{\eta})\}$ and $e^{\prime}=\{\omega(a)<\omega(a+\hat{\zeta}+\hat{\eta})\}$ with $a \in A_{p}$ and $\eta \in \mathcal{V} \backslash \mathcal{V}_{1}$, where $\beta \in \Sigma(\omega(a))$ denotes the simple transposition on $\{f(\zeta, a(\zeta)+1), f(\zeta, a(\zeta)+2)\}$. 
Finally, let us look at 2-simplices $\tau=\left\{\omega(a)<\omega\left(a+\hat{\eta}_{1}\right)<\omega\left(a+\hat{\eta}_{1}+\hat{\eta}_{2}\right)\right\}$ as in (vii). Recall that we have fixed a linear order on $\mathcal{V} \backslash \mathcal{V}_{1}$. In case $\eta_{1}<\eta_{2}$, one obtains

(8) $\pi\left(g_{e} g_{e^{\prime}}\right)=\pi\left(g_{e^{\prime \prime}}\right)$ for all $e=\left\{\omega(a)<\omega\left(a+\hat{\eta}_{1}\right)\right\}, e^{\prime}=\left\{\omega(a+\hat{\zeta})<\omega\left(a+\hat{\zeta}+\hat{\eta}_{2}\right)\right\}$ and $e^{\prime \prime}=\left\{\omega(a)<\omega\left(a+\hat{\eta}_{1}+\hat{\eta}_{2}\right)\right\}$ with $a \in A_{p}, \eta_{1}, \eta_{2} \in \mathcal{V} \backslash \mathcal{V}_{1}$ and $\eta_{1}<\eta_{2}$.

In case $\eta_{1}>\eta_{2}$, one obtains

(9) $\pi\left(\beta g_{e} g_{e^{\prime}} \beta\right)=\pi\left(g_{e^{\prime \prime}}\right)$ for all $e=\left\{\omega(a)<\omega\left(a+\hat{\eta}_{1}\right)\right\}, e^{\prime}=\left\{\omega(a+\hat{\zeta})<\omega\left(a+\hat{\zeta}+\hat{\eta}_{2}\right)\right\}$ and $e^{\prime \prime}=\left\{\omega(a)<\omega\left(a+\hat{\eta}_{1}+\hat{\eta}_{2}\right)\right\}$ with $a \in A_{p}, \eta_{1}, \eta_{2} \in \mathcal{V} \backslash \mathcal{V}_{1}$ and $\eta_{1}>\eta_{2}$, where $\beta \in$ $\Sigma(\omega(a))$ is the simple transposition on $\{f(\zeta, a(\zeta)+1), f(\zeta, a(\zeta)+2)\}$.

In case $\eta_{1}=\eta_{2}=\eta$, one has

(10) $\pi\left(g_{e} \sigma g_{e^{\prime}} \sigma\right)=\pi\left(g_{e^{\prime \prime}}\right)$ for all $e=\{\omega(a)<\omega(a+\hat{\eta})\}, e^{\prime}=\{\omega(a+\hat{\zeta})<\omega(a+\hat{\zeta}+\hat{\eta})\}$ and $e^{\prime \prime}=\{\omega(a)<\omega(a+2 \hat{\eta})\}$ with $a \in A_{p}, \eta \in \mathcal{V} \backslash \mathcal{V}_{1}$, where $\sigma \in \Sigma(\omega(a))$ is the simple transposition on $\{f(\eta, 1), f(\eta, 2)\}$.

For each $\eta \in \mathcal{V} \backslash \mathcal{V}_{1}$, consider the 1-simplex

$$
e=\{\omega((p+1) \hat{\zeta})<\omega((p+1) \hat{\zeta}+\hat{\eta})\} \in C_{1}^{\prime}
$$

and set $g_{\eta}=g_{e}$. Let $\Gamma_{0}$ be the free product of the groups $\Sigma(u)$ for $u \in C_{0}$ and the free group generated by $g_{\eta}$ for $\eta \in \mathcal{V} \backslash \mathcal{V}_{1}$. We regard $\Gamma_{0}$ as a subgroup of $\Gamma$. As a consequence of the discussion above, the following theorem is obtained.

Theorem 6.23. The homomorphism $\pi: \Gamma_{0} \rightarrow[[G \mid Y]]$ is surjective and its kernel is generated by the following elements as a normal subgroup.

- $i_{e}(\alpha) c_{e}\left(\alpha^{-1}\right)$ for any $e \in C_{1}$ and $\alpha \in \Sigma(e)$.

- $\left(\beta g_{\eta} \beta\right)^{-1} i_{e}(\alpha)\left(\beta g_{\eta} \beta\right) c_{e}\left(\alpha^{-1}\right)$ for any $e=\{\omega(a)<\omega(a+\hat{\eta})\} \in C_{1}^{\prime}$ and $\alpha \in \Sigma(e)$, where $a$ is in $A_{p} \cup A_{p+1}, \eta$ is in $\mathcal{V} \backslash \mathcal{V}_{1}$ and $\beta \in \Sigma(\omega(a))$ is the simple transposition on $\{f(\zeta, a(\zeta)+1), f(\zeta, p+2)\}$.

- $\left[g_{\eta_{1}}, \beta g_{\eta_{2}} \beta\right]$ for any distinct $\eta_{1}, \eta_{2} \in \mathcal{V} \backslash \mathcal{V}_{1}$, where $\beta \in \Sigma(\omega(p \hat{\zeta}))$ is the simple transposition on $\{f(\zeta, p+1), f(\zeta, p+2)\}$.

- $\left(\beta g_{\eta} \beta \sigma g_{\eta} \sigma\right)^{-1} i_{e}(\alpha)\left(\beta g_{\eta} \beta \sigma g_{\eta} \sigma\right) c_{e}\left(\alpha^{-1}\right)$ for any $e=\{\omega(p \hat{\zeta})<\omega(p \hat{\zeta}+2 \hat{\eta})\} \in C_{1}^{\prime}$ and $\alpha \in \Sigma(e)$, where $\eta$ is in $\mathcal{V} \backslash \mathcal{V}_{1}, \beta \in \Sigma(\omega(p \hat{\zeta}))$ denotes the simple transposition on $\{f(\zeta, p+1), f(\zeta, p+2)\}$ and $\sigma \in \Sigma(\omega(p \hat{\zeta}))$ denotes the simple transposition on $\{f(\eta, 1), f(\eta, 2)\}$.

As an application of the theorem above, we obtain the following.

Corollary 6.24. (1) The abelianization $[[G \mid Y]] / D([[G \mid Y]])$ of the topological full group $[[G \mid Y]]$ is isomorphic to $\left(H_{0}(G) \otimes \mathbb{Z}_{2}\right) \oplus H_{1}(G)$.

(2) The group $[[G \mid Y]]_{0} / D([[G \mid Y]])$ is isomorphic to $H_{0}(G) \otimes \mathbb{Z}_{2}$.

(3) The group $[[G \mid Y]]$ is simple if and only if $H_{0}(G)$ is 2-divisible. 
Proof. (1) Since $\Gamma_{0}$ is the free product of the groups $\Sigma(u)$ for $u \in C_{0}$ and the free group generated by $g_{\eta}$ for $\eta \in \mathcal{V} \backslash \mathcal{V}_{1}$, its abelianization $\Gamma_{0} / D\left(\Gamma_{0}\right)$ is isomorphic to

$$
\bigoplus_{u \in C_{0}}\left(\mathbb{Z}_{2}\right)^{\mathcal{V}} \oplus \mathbb{Z}^{\mathcal{V} \backslash \mathcal{V}_{1}}
$$

By the theorem above, we have $\pi\left(i_{e}(\alpha)\right)=\pi\left(c_{e}(\alpha)\right)$ for any $e=\{\omega(a)<\omega(a+\hat{\xi})\}$ and $\alpha \in \Sigma(e)$, where $a$ is in $A_{p} \cup A_{p+1}$ and $\xi$ is in $\mathcal{V}_{1}$. These equations collapse the group $\Gamma_{0} / D\left(\Gamma_{0}\right)$ to

$$
\left(\mathbb{Z}_{2}\right)^{\mathcal{V}} \oplus \mathbb{Z}^{\mathcal{V} \backslash \mathcal{V}_{1}}
$$

By the theorem above, we also have $\pi\left(i_{e}(\alpha)\right)=\pi\left(c_{e}(\alpha)\right)$ for any $e=\{\omega(a)<\omega(a+2 \hat{\xi})\}$ and $\alpha \in \Sigma(e)$, where $a$ is in $A_{p}$ and $\xi$ is in $\mathcal{V}_{1}$. These equations collapse the group above to

$$
\left(\left(\mathbb{Z}_{2}\right)^{\mathcal{V}} / \operatorname{Im}\left(\mathrm{id}-M^{t}\right)\right) \oplus \mathbb{Z}^{\mathcal{V} \backslash \mathcal{V}_{1}}
$$

which is isomorphic to $\left(H_{0}(G) \otimes \mathbb{Z}_{2}\right) \oplus H_{1}(G)$. The theorem above tells us that no further collapsing occurs.

(2) This follows from (1) and Theorem 5.2.

(3) As mentioned in Section 6.1, $H_{0}(G)$ is a finitely generated abelian group and $H_{1}(G)$ is isomorphic to the torsion-free part of $H_{0}(G)$. Therefore if $H_{0}(G)$ is 2-divisible, then $\left(H_{0}(G) \otimes \mathbb{Z}_{2}\right) \oplus H_{1}(G)=0$, and so $[[G \mid Y]]=D([[G \mid Y]])$. By Lemma 6.1 and Theorem 4.16, $D([[G \mid Y]])$ is simple. Hence $[[G \mid Y]]$ is simple. The other implication is shown in a similar way.

The corollary above, together with Theorem 6.11 and Theorem 6.21, implies the following.

Corollary 6.25. (1) The group $D([[G \mid Y]])$ is finitely generated.

(2) If $H_{0}(G)$ is a finite group, then the groups $[[G \mid Y]]_{0}$ and $D([[G \mid Y]])$ are of type $F_{\infty}$. In particular, they are finitely presented and are of type $F P_{\infty}$.

In general, we do not know if $[[G \mid Y]]_{0}$ and $D([[G \mid Y]])$ are finitely presented or not.

\subsection{Examples}

\subsubsection{Full shifts}

We consider étale groupoids arising from full shifts. Let $n \in \mathbb{N} \backslash\{1\}$ and let $r \in \mathbb{N}$. Let $(\mathcal{V}, \mathcal{E})$ be a finite directed graph such that $\# \mathcal{V}=r$ and its adjacency matrix is

$$
M=\left[\begin{array}{ccccc}
0 & 0 & \ldots & 0 & n \\
1 & 0 & \ldots & 0 & 0 \\
0 & 1 & \ldots & 0 & 0 \\
\vdots & \vdots & \ddots & \vdots & \vdots \\
0 & 0 & \ldots & 1 & 0
\end{array}\right]
$$


Note that $\operatorname{det}\left(\mathrm{id}-M^{t}\right)=1-n$. Let $\left(X_{n, r}, \sigma_{n, r}\right)$ be the shift of finite type associated with $(\mathcal{V}, \mathcal{E})$. When $r=1$, the shift $\left(X_{n, 1}, \sigma_{n, 1}\right)$ is the full shift over $n$ symbols. Let $G_{n, r}$ be the étale groupoid of the shift $\left(X_{n, r}, \sigma_{n, r}\right)$ (see Section 6.1). One has

$$
H_{0}\left(G_{n, r}\right) \cong \operatorname{Coker}\left(\mathrm{id}-M^{t}\right) \cong \mathbb{Z}_{n-1} \quad \text { and } \quad H_{1}\left(G_{n, r}\right) \cong \operatorname{Ker}\left(\mathrm{id}-M^{t}\right)=0,
$$

where id $-M^{t}$ is thought of as a homomorphism from $\mathbb{Z}^{r}$ to $\mathbb{Z}^{r}$. The equivalence class of $1_{X_{n, r}}$ in $H_{0}\left(G_{n, r}\right)$ corresponds to $r \in \mathbb{Z}_{n-1}$. As mentioned in Remark 6.3, the topological full group $\left[\left[G_{n, r}\right]\right]$ is naturally isomorphic to the Higman-Thompson group $V_{n, r}$. From $H_{1}\left(G_{n, r}\right)=0$, we obtain $\left[\left[G_{n, r}\right]\right]=\left[\left[G_{n, r}\right]\right]_{0}$. By Lemma 6.1 and Theorem 4.16, $D\left(\left[\left[G_{n, r}\right]\right]\right)$ is simple. Moreover, Corollary 6.24 says that

$$
\left[\left[G_{n, r}\right]\right] / D\left(\left[\left[G_{n, r}\right]\right]\right) \cong \mathbb{Z}_{n-1} \otimes \mathbb{Z}_{2}= \begin{cases}0 & n \text { is even } \\ \mathbb{Z}_{2} & n \text { is odd }\end{cases}
$$

By Theorem 3.9 and Theorem 6.2 , $\left[\left[G_{n, r}\right]\right]$ (or $D\left(\left[\left[G_{n, r}\right]\right]\right)$ ) is isomorphic to $\left[\left[G_{m, s}\right]\right]$ (or $\left.D\left(\left[\left[G_{m, s}\right]\right]\right)\right)$ if and only if there exists an isomorphism $\varphi: \mathbb{Z}_{n-1} \rightarrow \mathbb{Z}_{m-1}$ such that $\varphi(r)=s$. This reproves the main result of [36]. By Theorem 6.21 and Corollary 6.25, $\left[\left[G_{n, r}\right]\right]$ and $D\left(\left[\left[G_{n, r}\right]\right]\right)$ are of type $\mathrm{F}_{\infty}$.

\subsubsection{The golden mean shift}

Let $X$ be the set of sequences of $\{0,1\}$ in which ' 11 ' does not appear, that is,

$$
X=\left\{\left(x_{n}\right)_{n} \in\{0,1\}^{\mathbb{N}} \mid \text { if } x_{n}=1, \text { then } x_{n+1}=0\right\} .
$$

Let $\sigma: X \rightarrow X$ be the one-sided shift. The dynamical system $(X, \sigma)$ is called the golden mean shift, and the corresponding matrix is

$$
M=\left[\begin{array}{ll}
1 & 1 \\
1 & 0
\end{array}\right]
$$

The Perron eigenvalue of $M$ is the golden number $(1+\sqrt{5}) / 2$, and $\operatorname{det}\left(\mathrm{id}-M^{t}\right)=-1$. Consider the étale groupoid $G$ of $(X, \sigma)$. One has

$$
H_{0}(G) \cong \operatorname{Coker}\left(\mathrm{id}-M^{t}\right)=0 \quad \text { and } \quad H_{1}(G) \cong \operatorname{Ker}\left(\mathrm{id}-M^{t}\right)=0 .
$$

It follows from Theorem 6.2 that $G$ is isomorphic to $G_{2,1}$ discussed above. Hence $[[G]]$ is isomorphic to the Higman-Thompson group $V_{2,1}$.

In 24, for an arbitrary real number $\beta>1$, the $\beta$-shifts and the associated $C^{*}$-algebras $\mathcal{O}_{\beta}$ were studied. It is known that the $\beta$-shift is a shift of finite type if and only if the $\beta$-expansion of 1 is finite (see [24, Proposition 3.8] for instance). The golden mean shift $(X, \sigma)$ is equal to the $\beta$-shift with $\beta=(1+\sqrt{5}) / 2$. Note that the ring $\mathbb{Z}\left[\beta, \beta^{-1}\right]$ equals $\mathbb{Z}+\beta \mathbb{Z}$, because $\beta^{2}-\beta-1=0$. We would like to observe that $[[G]]$ is identified with a group of PL bijections on the unit interval. As in Remark 6.3, let $F_{\beta, 1}$ be the set of all PL homeomorphisms $f:[0,1] \rightarrow[0,1]$ with finitely many singularities such that all singularities of $f$ are in $\mathbb{Z}\left[\beta, \beta^{-1}\right]$ and the derivative of $f$ at any non-singular point is $\beta^{k}$ for some $k \in \mathbb{Z}$. The group $F_{\beta, 1}$ was shown to be finitely presented and is of type $\mathrm{FP}_{\infty}$ 
in [8]. In the same fashion as Remark 6.3, one can define the group $V_{\beta, 1}$ containing $F_{\beta, 1}$. Define a continuous map $\rho: X \rightarrow[0,1]$ by

$$
\rho\left(\left(x_{n}\right)_{n}\right)=\sum_{n=1}^{\infty} \frac{x_{n}}{\beta^{n}} \quad \forall\left(x_{n}\right)_{n} \in X .
$$

Then for every $t \in[0,1]$,

$$
\# \rho^{-1}(t)= \begin{cases}2 & t \in \mathbb{Z}\left[\beta, \beta^{-1}\right] \cap[0,1) \\ 1 & \text { otherwise }\end{cases}
$$

Any cylinder set of $X$ is mapped to an interval $[a, b]$ with $a, b \in \mathbb{Z}\left[\beta, \beta^{-1}\right]$. It is not so hard to see that $[[G]]$ is naturally isomorphic to a subgroup of $V_{\beta, 1}$ via the map $\rho$. Thus $V_{2,1}$ is embeddable into $V_{\beta, 1}$.

\subsubsection{A subshift with non-trivial $H_{1}$}

Let $(X, \sigma)$ be the irreducible shift of finite type arising from the matrix

$$
M=\left[\begin{array}{ll}
2 & 1 \\
1 & 2
\end{array}\right]
$$

and let $G$ be the étale groupoid of $(X, \sigma)$. One has

$$
H_{0}(G) \cong \operatorname{Coker}\left(\mathrm{id}-M^{t}\right) \cong \mathbb{Z} \quad \text { and } \quad H_{1}(G) \cong \operatorname{Ker}\left(\mathrm{id}-M^{t}\right)=\mathbb{Z}
$$

The equivalence class of $1_{X}$ in $H_{0}(G)$ corresponds to $0 \in \mathbb{Z}$. By Lemma 6.1 and Theorem 4.16, $D([[G]])$ is simple. Theorem $\left[5.2\right.$ says that $[[G]] /[[G]]_{0} \cong \mathbb{Z}$. By Corollary $[6.24$, $[[G]]_{0} / D([[G]]) \cong \mathbb{Z}_{2}$. By Theorem 6.21, $[[G]]$ is of type $\mathrm{F}_{\infty}$. By Theorem 6.11 and Corollary 6.25 , $[[G]]_{0}$ and $D([[G]])$ are finitely generated.

We would like to give a finite presentation for $[[G]]$. The notation is borrowed from Section 6.5 and Section 6.6. Let $(\mathcal{V}, \mathcal{E})$ be a finite directed graph whose adjacency matrix is $M$. Let $\mathcal{V}=\{\zeta, \eta\}$. Then $\omega=\left\{D_{\zeta}, D_{\eta}\right\}$ is in $\mathcal{B}$ and $\operatorname{rank}(\omega)=(1,1)$. It is easy to see $\operatorname{rank}(u)=(n, n)$ for $u \in \mathcal{B}_{\omega}$ with height $(u)=n-1$. By Lemma 6.18, there exists $n$ such that $K_{u}$ is 1 -connected for any $u \in \mathcal{B}_{\omega}$ satisfying $\operatorname{rank}(u)=(n, n)$. In view of the remark given in [5, Section 5] (this argument is due to K. Vogtmann), one can take $n=9$ in this case. Thus, the simplicial complex $Z_{5,7}$ is 1 -connected. Let $u_{6} \in \mathcal{B}_{\omega}$ be such that $\operatorname{rank}\left(u_{6}\right)=(6,6)$. Choose two elements $V_{1}, V_{2} \in u_{6}$ such that $s\left(V_{1}\right)=s\left(V_{2}\right)=D_{\zeta}$. Let $u_{7}$ be the elementary expansion of $u_{6}$ with respect to $V_{1}$ and let $u_{8}$ be the elementary expansion of $u_{7}$ with respect to $V_{2}$. Then $\operatorname{rank}\left(u_{k}\right)=(k, k),\left\{u_{6}<u_{7}<u_{8}\right\}$ is a 2-simplex of $Z_{5,7}$ and $\left\{u_{6}, u_{7}, u_{8}\right\}$ is a set of representatives for the vertices of $Z_{5,7} \bmod [[G]]$. The stabilizer of $u_{k}$ is isomorphic to $\Sigma_{k} \times \Sigma_{k}$ for each $k=6,7,8$. To simplify notation, we denote products of symmetric groups by $\Sigma_{k, l}=\Sigma_{k} \times \Sigma_{l}$, etc. Let $\Gamma_{0}$ be the free product of $\Sigma_{6,6}, \Sigma_{7,7}, \Sigma_{8,8}$ and $\mathbb{Z}$ generated by $g=g_{\eta}$. As discussed in Section 6.6, there exists a natural surjection $\pi: \Gamma_{0} \rightarrow[[G]]$. 
Consider the direct limit (in the sense of J-P. Serre) of the following diagram.

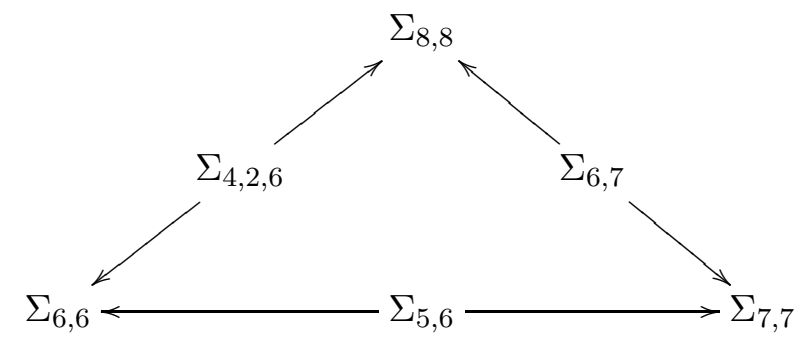

Regard $\Sigma_{k, k}$ as the group of permutations of letters $\left\{1_{k}, 2_{k}, \ldots, k_{k}\right\} \cup\left\{1_{k}^{\prime}, 2_{k}^{\prime}, \ldots, k_{k}^{\prime}\right\}$ for $k=6,7,8$. The maps $\Sigma_{5,6} \hookrightarrow \Sigma_{k, k}$ for $k=6,7$ are obtained by letting $\Sigma_{5}$ permute $\left\{1_{k}, 2_{k}, 3_{k}, 4_{k}, k_{k}\right\}$ and letting $\Sigma_{6}$ permute $\left\{1_{k}^{\prime}, 2_{k}^{\prime}, \ldots, 6_{k}^{\prime}\right\}$. Similarly, the maps $\Sigma_{6,7} \hookrightarrow \Sigma_{k, k}$ for $k=7,8$ are obtained by letting $\Sigma_{6}$ permute $\left\{1_{k}, \ldots, 6_{k}\right\}$ and letting $\Sigma_{7}$ permute $\left\{1_{k}^{\prime}, \ldots, 7_{k}^{\prime}\right\}$. The map $\Sigma_{4,2,6} \hookrightarrow \Sigma_{6,6}$ is obtained by letting $\Sigma_{4,2,6}$ permute $\left\{1_{6}, \ldots, 4_{6}\right\}$, $\left\{5_{6}, 6_{6}\right\}$ and $\left\{1_{6}^{\prime}, \ldots, 6_{6}^{\prime}\right\}$. The embedding $\Sigma_{4,2,6} \hookrightarrow \Sigma_{8,8}$ is not of the standard type. The subgroup $\Sigma_{4} \times \Sigma_{6}$ permutes $\left\{1_{8}, \ldots, 4_{8}\right\}$ and $\left\{1_{8}^{\prime}, \ldots, 6_{8}^{\prime}\right\}$, but the non-trivial element of $\Sigma_{2}$ maps to the product $\left(5_{8} 7_{8}\right)\left(6_{8} 8_{8}\right)\left(7_{8}^{\prime} 8_{8}^{\prime}\right)$ of three transpositions. Let $\Gamma_{1}$ be the direct limit of this triangle of groups. Then the restriction of the homomorphism $\pi: \Gamma_{0} \rightarrow[[G]]$ to the subgroup $\Sigma_{6,6} * \Sigma_{7,7} * \Sigma_{8,8}$ factors through $\Gamma_{1}$.

Let us now turn to discussion on the relations involving $g=g_{\eta}$. We consider the following diagram.

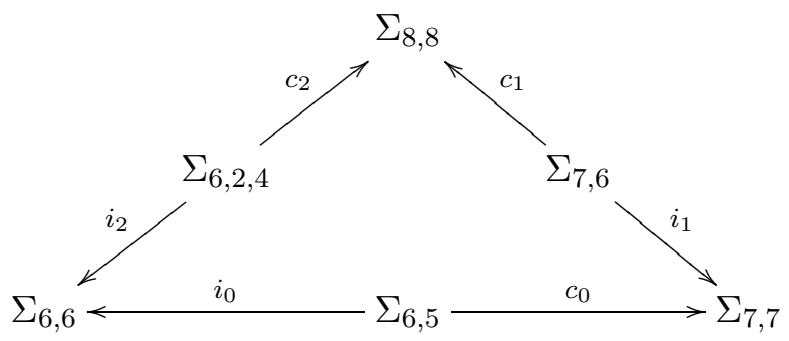

The maps $i_{0}$ and $c_{0}$ are obtained by letting $\Sigma_{6}$ permute $\left\{1_{k}, \ldots, 5_{k}, k_{k}\right\}$ and letting $\Sigma_{5}$ permute $\left\{2_{k}^{\prime}, \ldots, 6_{k}^{\prime}\right\}$ for $k=6,7$. The maps $i_{1}$ and $c_{1}$ are obtained by letting $\Sigma_{7}$ permute $\left\{1_{k}, \ldots, 7_{k}\right\}$ and letting $\Sigma_{6}$ permute $\left\{2_{k}^{\prime}, \ldots, 7_{k}^{\prime}\right\}$ for $k=7,8$. The map $i_{2}$ is obtained by letting $\Sigma_{6,2,4}$ permute $\left\{1_{6}, \ldots, 6_{6}\right\},\left\{1_{6}^{\prime}, 2_{6}^{\prime}\right\}$ and $\left\{3_{6}^{\prime}, \ldots, 6_{6}^{\prime}\right\}$. The map $c_{2}$ is not of the standard type. The subgroup $\Sigma_{6} \times \Sigma_{4}$ permutes $\left\{1_{8}, \ldots, 5_{8}, 7_{8}\right\}$ and $\left\{3_{8}^{\prime}, \ldots, 6_{8}^{\prime}\right\}$, but the non-trivial element of $\Sigma_{2}$ maps to the product $\left(1_{8}^{\prime} 2_{8}^{\prime}\right)\left(7_{8}^{\prime} 8_{8}^{\prime}\right)\left(6_{8} 8_{8}\right)$ of three transpositions. Now the relations involving $g$ are given by

$$
\left(\beta_{0} g \beta_{0}\right)^{-1} i_{0}(\sigma)\left(\beta_{0} g \beta_{0}\right) c_{0}(\sigma) \quad \forall \sigma \in \Sigma_{6,5}, \quad g^{-1} i_{1}(\sigma) g c_{1}(\sigma) \quad \forall \sigma \in \Sigma_{7,6}
$$

and

$$
\left(\beta_{0} g \beta_{0} \beta_{1} g \beta_{1}\right)^{-1} i_{2}(\sigma)\left(\beta_{0} g \beta_{0} \beta_{1} g \beta_{1}\right) c_{2}(\sigma) \quad \forall \sigma \in \Sigma_{6,2,4},
$$

where $\beta_{0}=\left(\begin{array}{ll}5_{6} & 6_{6}\end{array}\right)$ and $\beta_{1}=\left(1_{6}^{\prime} 2_{6}^{\prime}\right)$. Hence $[[G]]$ is isomorphic to the quotient of the free product of $\Gamma_{1}$ and $\mathbb{Z}=\langle g\rangle$ by the normal subgroup generated by these relations. 


\subsubsection{Boundary actions I}

We consider the boundary actions of free products of finite groups. These actions and related $C^{*}$-algebras were studied in $[40,35$. Let $P$ and $Q$ be non-trivial finite groups (we exclude the case $P=Q=\mathbb{Z}_{2}$ ). Put $p=\# P$ and $q=\# Q$. Let $\mathcal{E}$ be the disjoint union of $P \backslash\{1\}$ and $Q \backslash\{1\}$. We define

$$
X=\left\{\left(x_{n}\right)_{n} \in \mathcal{E}^{\mathbb{N}} \mid \text { if } x_{n} \in P\left(\text { resp. } x_{n} \in Q\right) \text {, then } x_{n+1} \in Q\left(\text { resp. } x_{n+1} \in P\right)\right\} .
$$

The topological space $X$ is naturally identified with the hyperbolic boundary of the free product $P * Q$, and admits a natural action $\varphi: P * Q \curvearrowright X$ by homeomorphisms, which is called the boundary action (see [35, Proposition 5.5], for example). Let $\sigma: X \rightarrow X$ be the one-sided shift. Then $(X, \sigma)$ is the shift of finite type associated with the matrix

$$
M=\left[\begin{array}{cc}
0 & p-1 \\
q-1 & 0
\end{array}\right] .
$$

We have $\operatorname{det}\left(\mathrm{id}-M^{t}\right)=1-(p-1)(q-1)$. It is not so hard to see that the transformation groupoid $G_{\varphi}$ is canonically identified with the étale groupoid arising from $(X, \sigma)$ (see [31, Definition 2.1] for the definition of $\left.G_{\varphi}\right)$. By Lemma 6.1, the groupoid $G_{\varphi}$ is purely infinite and minimal. One has

$$
H_{0}\left(G_{\varphi}\right) \cong \operatorname{Coker}\left(\mathrm{id}-M^{t}\right) \cong \mathbb{Z}_{n-1} \quad \text { and } \quad H_{1}\left(G_{\varphi}\right) \cong \operatorname{Ker}\left(\mathrm{id}-M^{t}\right)=0,
$$

where $n=(p-1)(q-1)$. The equivalence class of $1_{X}$ in $H_{0}\left(G_{\varphi}\right)$ corresponds to $p \in \mathbb{Z}_{n-1}$ (or $q \in \mathbb{Z}_{n-1}$ ). Hence Theorem 6.2 tells us that $G_{\varphi}$ is isomorphic to $G_{n, p}$ (or $G_{n, q}$ ) discussed in Section 6.7.1.

\subsubsection{Boundary actions II}

Let $k \in \mathbb{N} \backslash\{1\}$ and let $\mathbb{F}_{k}$ denote the free group on $k$ generators $s_{1}, s_{2}, \ldots, s_{k}$. Let $\mathcal{E}=\left\{s_{1}, s_{1}^{-1}, s_{2}, s_{2}^{-1}, \ldots, s_{k}, s_{k}^{-1}\right\}$. Set

$$
X_{k}=\left\{\left(x_{n}\right)_{n} \in \mathcal{E}^{\mathbb{N}} \mid \text { if } x_{n}=s_{i}\left(\text { resp. } x_{n}=s_{i}^{-1}\right) \text {, then } x_{n+1} \neq s_{i}^{-1}\left(\text { resp. } x_{n+1} \neq s_{i}\right)\right\} .
$$

In the same way as the preceding example, the space $X_{k}$ is naturally identified with the hyperbolic boundary of the free group $\mathbb{F}_{k}$. Let $\varphi_{k}: \mathbb{F}_{k} \curvearrowright X_{k}$ be the boundary action. The one-sided shift $\sigma_{k}: X_{k} \rightarrow X_{k}$ is a shift of finite type associated with the $2 k \times 2 k$ matrix

$$
M=\left[\begin{array}{cccccccc}
1 & 0 & 1 & 1 & \ldots & \ldots & 1 & 1 \\
0 & 1 & 1 & 1 & \ldots & \ldots & 1 & 1 \\
1 & 1 & 1 & 0 & \ldots & \ldots & 1 & 1 \\
1 & 1 & 0 & 1 & \ldots & \ldots & 1 & 1 \\
\vdots & \vdots & \vdots & \vdots & \ddots & & \vdots & \vdots \\
\vdots & \vdots & \vdots & \vdots & & \ddots & \vdots & \vdots \\
1 & 1 & 1 & 1 & \ldots & \ldots & 1 & 0 \\
1 & 1 & 1 & 1 & \ldots & \ldots & 0 & 1
\end{array}\right] .
$$


In the same way as the preceding example, the transformation groupoid $G_{\varphi_{k}}$ is canonically identified with the étale groupoid arising from $\left(X_{k}, \sigma_{k}\right)$. By Lemma 6.1, the groupoid $G_{\varphi_{k}}$ is purely infinite and minimal. We have

$$
H_{0}\left(G_{\varphi_{k}}\right) \cong \operatorname{Coker}\left(\mathrm{id}-M^{t}\right) \cong \mathbb{Z}^{k} \oplus \mathbb{Z}_{k-1}
$$

and

$$
H_{1}\left(G_{\varphi_{k}}\right) \cong \operatorname{Ker}\left(\mathrm{id}-M^{t}\right) \cong \mathbb{Z}^{k}
$$

The equivalence class of $1_{X}$ in $H_{0}\left(G_{\varphi_{k}}\right)$ corresponds to $(0,1) \in \mathbb{Z}^{k} \oplus \mathbb{Z}_{k-1}$. By Theorem [3.9, if $k \neq l$, then $\left[\left[G_{\varphi_{k}}\right]\right]$ (or $\left[\left[G_{\varphi_{k}}\right]\right]_{0}, D\left(\left[\left[G_{\varphi_{k}}\right]\right]\right)$ ) is not isomorphic to $\left[\left[G_{\varphi_{l}}\right]\right]$ (or $\left.\left[\left[G_{\varphi_{l}}\right]\right]_{0}, D\left(\left[\left[G_{\varphi_{l}}\right]\right]\right)\right)$. It follows from Theorem 5.2 that $\left[\left[G_{\varphi_{k}}\right]\right] /\left[\left[G_{\varphi_{k}}\right]\right]_{0}$ is isomorphic to $\mathbb{Z}^{k}$. Theorem 4.16 tells us that $D\left(\left[\left[G_{\varphi_{k}}\right]\right]\right)$ is simple. By Corollary 6.24 ,

$$
\left[\left[G_{\varphi_{k}}\right]\right]_{0} / D\left(\left[\left[G_{\varphi_{k}}\right]\right]\right) \cong\left(\mathbb{Z}^{k} \oplus \mathbb{Z}_{k-1}\right) \otimes \mathbb{Z}_{2}= \begin{cases}\left(\mathbb{Z}_{2}\right)^{k} & k \text { is even } \\ \left(\mathbb{Z}_{2}\right)^{k+1} & k \text { is odd }\end{cases}
$$

By Theorem 6.21, we know that $\left[\left[G_{\varphi_{k}}\right]\right]$ is of type $\mathrm{F}_{\infty}$. By Theorem 6.11 and Corollary 6.25, $\left[\left[G_{\varphi_{k}}\right]\right]_{0}$ and $D\left(\left[\left[G_{\varphi_{k}}\right]\right]\right)$ are finitely generated.

\section{Acknowledgments}

I am very much indebted to D. Tamaki for helpful discussions on homotopy theory and algebraic topology. I also thank R. Grigorchuk and K. Matsumoto for many valuable suggestions and comments.

\section{References}

[1] S. Bezuglyi and K. Medynets, Full groups, flip conjugacy, and orbit equivalence of Cantor minimal systems, Colloq. Math. 110 (2008), 409-429.

[2] K. S. Brown, Cohomology of groups, Graduate Texts in Mathematics, 87. SpringerVerlag, New York-Berlin, 1982.

[3] K. S. Brown, Presentations for groups acting on simply-connected complexes, J. Pure Appl. Algebra 32 (1984), 1-10.

[4] K. S. Brown, Finiteness properties of groups, J. Pure Appl. Algebra 44 (1987), 45-75.

[5] K. S. Brown, The geometry of finitely presented infinite simple groups, Algorithms and classification in combinatorial group theory (Berkeley, CA, 1989), 121-136, Math. Sci. Res. Inst. Publ., 23, Springer, New York, 1992.

[6] K. S. Brown and R. Geoghegan, An infinite-dimensional torsion-free $F P_{\infty}$ group, Invent. Math. 77 (1984), 367-381.

[7] J. W. Cannon, W. J. Floyd and W. R. Parry, Introductory notes on Richard Thompson's groups, Enseign. Math. (2) 42 (1996), 215-256.

[8] S. Cleary, Regular subdivision in $\mathbb{Z}[(1+\sqrt{5}) / 2]$, Illinois J. Math. 44 (2000), 453-464. 
[9] J. Cuntz, A class of $C^{*}$-algebras and topological Markov chains II: Reducible chains and the Ext-functor for $C^{*}$-algebras, Invent. Math. 63 (1981), 25-40.

[10] J. Cuntz and W. Krieger, A class of $C^{*}$-algebras and topological Markov chains, Invent. Math. 56 (1980), 251-268.

[11] H. A. Dye, On groups of measure preserving transformations I, Amer. J. Math. 81 (1959), 119-159.

[12] H. A. Dye, On groups of measure preserving transformations II, Amer. J. Math. 85 (1963), 551-576.

[13] J. Franks, Flow equivalence of subshifts of finite type, Ergodic Theory Dynam. Systems 4 (1984), 53-66.

[14] D. H. Fremlin, Measure theory Vol. 3 Measure algebras, Torres Fremlin, Colchester, 2004 .

[15] R. Geoghegan, Topological methods in group theory, Graduate Texts in Mathematics 243. Springer, New York, 2008.

[16] T. Giordano, H. Matui, I. F. Putnam and C. F. Skau, Orbit equivalence for Cantor minimal $\mathbb{Z}^{d}$-systems, Invent. Math. 179 (2010), 119-158. arXiv:0810.3957

[17] T. Giordano, I. F. Putnam and C. F. Skau, Full groups of Cantor minimal systems, Israel J. Math. 111 (1999), 285-320.

[18] R. Grigorchuk and K. Medynets, On algebraic properties of topological full groups, preprint. arXiv:1105.0719

[19] P. de la Harpe, Topics in geometric group theory, Chicago Lectures in Mathematics, University of Chicago Press, Chicago, IL, 2000.

[20] G. Higman, Finitely presented infinite simple groups, Notes on Pure Mathematics, No. 8 (1974), Australian National University, Canberra, 1974.

[21] B. Hughes, Local similarities and the Haagerup property, with an appendix by Daniel S. Farley, Groups Geom. Dyn. 3 (2009), 299-315. arXiv:0804.0610

[22] P. Jolissaint and G. Robertson, Simple purely infinite $C^{*}$-algebras and $n$-filling actions, J. Funct. Anal. 175 (2000), 197-213.

[23] K. Juschenko and N. Monod, Cantor systems, piecewise translations and simple amenable groups, preprint. arXiv:1204.2132

[24] Y. Katayama, K. Matsumoto and Y. Watatani, Simple $C^{*}$-algebras arising from $\beta$ expansion of real numbers, Ergodic Theory Dynam. Systems 18 (1998), 937-962.

[25] D. Lind and B. Marcus, An introduction to symbolic dynamics and coding, Cambridge University Press, Cambridge, 1995. 
[26] K. Matsumoto, Orbit equivalence of topological Markov shifts and Cuntz-Krieger algebras, Pacific J. Math. 246 (2010), 199-225. arXiv:0707.2114

[27] K. Matsumoto, Classification of Cuntz-Krieger algebras by orbit equivalence of topological Markov shifts, to appear in Proc. Amer. Math. Soc.

[28] K. Matsumoto, K-groups of the full group actions on one-sided topological Markov shifts, to appear in Discrete Contin. Dyn. Syst.

[29] K. Matsumoto, Full groups of one-sided topological Markov shifts, preprint. arXiv: 1205.1320

[30] H. Matui, Some remarks on topological full groups of Cantor minimal systems, Internat. J. Math. 17 (2006), 231-251. math.DS/0404117

[31] H. Matui, Homology and topological full groups of étale groupoids on totally disconnected spaces, Proc. Lond. Math. Soc. (3) 104 (2012), 27-56. arXiv:0909.1624

[32] H. Matui, Some remarks on topological full groups of Cantor minimal systems II, to appear in Ergodic Theory Dynam. Systems. arXiv:1111.3134

[33] K. Medynets, Reconstruction of orbits of Cantor systems from full groups, Bull. London Math. Soc. 43 (2011) 1104-1110. arXiv:1006.1145

[34] V. V. Nekrashevych, Cuntz-Pimsner algebras of group actions, J. Operator Theory 52 (2004), 223-249.

[35] R. Okayasu, Cuntz-Krieger-Pimsner algebras associated with amalgamated free product groups, Publ. Res. Inst. Math. Sci. 38 (2002), 147-190. arXiv:math/0010097

[36] E. Pardo, The isomorphism problem for Higman-Thompson groups, J. Algebra 344 (2011), 172-183. arXiv:1006.1759

[37] J. Renault, A groupoid approach to $C^{*}$-algebras, Lecture Notes in Mathematics 793, Springer, Berlin, 1980.

[38] J. Renault, Cartan subalgebras in $C^{*}$-algebras, Irish Math. Soc. Bull. 61 (2008), 29-63. arXiv:0803.2284

[39] M. Rørdam and A. Sierakowski, Purely infinite $C^{*}$-algebras arising from crossed products, Ergodic Theory Dynam. Systems 32 (2012), 273-293.

[40] J. Spielberg, Free-product groups, Cuntz-Krieger algebras, and covariant maps, Internat. J. Math. 2 (1991), 457-476.

[41] M. Stein, Groups of piecewise linear homeomorphisms, Trans. Amer. Math. Soc. 332 (1992), 477-514. 